This item was submitted to Loughborough's Research Repository by the author.

Items in Figshare are protected by copyright, with all rights reserved, unless otherwise indicated.

\title{
A bifurcation theory for three-dimensional oblique travelling gravity-capillary water waves
}

PLEASE CITE THE PUBLISHED VERSION

LICENCE

CC BY-NC-ND 4.0

\section{REPOSITORY RECORD}

Groves, Mark D., and M. Haragus. 2019. "A Bifurcation Theory for Three-dimensional Oblique Travelling Gravity-capillary Water Waves". figshare. https://hdl.handle.net/2134/457. 


\title{
A bifurcation theory for three-dimensional oblique travelling gravity-capillary water waves
}

\author{
M. D. Groves \\ Department of Mathematical Sciences \\ Loughborough University \\ Loughborough \\ LE11 3TU \\ $U K$ \\ M. Haragus \\ Mathématiques Appliquées de Bordeaux \\ Université Bordeaux I \\ 351, Cours de la Libération \\ 33405 Talence Cedex \\ France
}

\begin{abstract}
This article presents a rigorous existence theory for small-amplitude three-dimensional travelling water waves. The hydrodynamic problem is formulated as an infinite-dimensional Hamiltonian system in which an arbitrary horizontal spatial direction is the time-like variable. Wave motions which are periodic in a second, different horizontal direction are detected using a centre-manifold reduction technique by which the problem is reduced to a locally equivalent Hamiltonian system with a finite number of degrees of freedom.

A catalogue of bifurcation scenarios is compiled by means of a geometric argument based upon the classical dispersion relation for travelling water waves. Taking all parameters into account, one finds that this catalogue includes virtually any bifurcation or resonance known in Hamiltonian systems theory. Nonlinear bifurcation theory is carried out for a representative selection of bifurcation scenarios; solutions of the reduced Hamiltonian system are found by applying results from the well-developed theory of finite-dimensional Hamiltonian systems such as the Lyapunov centre theorem and the Birkhoff normal form.

We find oblique line waves which depend only upon one spatial direction which is not aligned with the direction of wave propagation; the waves have periodic, solitary-wave or generalised solitary-wave profiles in this distinguished direction. Truly three-dimensional waves are also found which have periodic, solitary-wave or generalised solitary-wave profiles in one direction and are periodic in another. In particular, we recover doubly periodic waves with arbitrary fundamental domains and oblique versions of the results on threedimensional travelling waves already in the literature.
\end{abstract}




\section{Introduction}

This article is concerned with the classical three-dimensional gravity-capillary water-wave problem in hydrodynamics, namely the three-dimensional irrotational flow of a perfect fluid of unit density subject to the forces of gravity and surface tension. The fluid motion is governed by the Euler equations in a domain bounded below by a rigid horizontal bottom and above by a free surface which is described as a graph $\{Y=h+\eta(X, Z, t)\}$, where $h$ represents the depth of the fluid in its undisturbed state and the function $\eta$ depends upon the two unbounded horizontal directions $X$ and $Z$ and time $t$. In this paper we construct a wide variety of small-amplitude travelling gravity-capillary waves, that is waves which are uniformly translating in a distinguished horizontal direction. Specifically, we study waves which travel with constant speed $c$ in the $X$ direction, have qualitatively prescribed profiles in an arbitrary horizontal direction $x$ forming an angle $\theta_{1}$ with the $X$-axis and are periodic in another horizontal direction $z$ forming an angle $\theta_{2}$ with the $X$-axis (see Figure 1). These waves are special solutions of the Euler equations in which the free surface takes the form

$$
Y=h+\eta(x, z)
$$

where

$$
x=\csc \left(\theta_{2}-\theta_{1}\right)\left[(X-c t) \sin \theta_{2}-Z \cos \theta_{2}\right], \quad z=\csc \left(\theta_{1}-\theta_{2}\right)\left[(X-c t) \sin \theta_{1}-Z \cos \theta_{1}\right]
$$

and the function $\eta$ is periodic in its second argument; we refer to such solutions as oblique travelling waves since their main features are not necessarily aligned with their direction of propagation. The Euler equations for oblique travelling gravity-capillary water waves are stated in full in Section 2.1 below.

In contrast to the extensively studied two-dimensional travelling water-wave problem involving only one horizontal coordinate, relatively few results are available concerning threedimensional travelling waves. Indeed, most of the existing literature deals with model equations in either the long- or the short-wave approximation; a comprehensive survey is given in the review papers by Aklyas [1] and Dias \& Kharif [8]. There are several rigorous existence results for three-dimensional travelling gravity-capillary water waves whose free surface is doubly periodic, for example the 'hexagonal' wave patterns observed in nature and predicted on the basis of model equations (Hammack, Scheffner \& Segur [12]) and the 'short-crested waves' whose fundamental domain is a 'symmetric diamond' (doubly periodic waves with equal periods). Existence of the latter waves was established by Reeder \& Shinbrot [29], and Craig \& Nicholls [5] have recently given an existence theory for waves with arbitrary fundamental domain using a variational Lyapunov-Schmidt reduction of the Euler equations.

The existence question for three-dimensional travelling water waves which are periodic in only one horizontal direction was first considered by Groves \& Mielke [11], who considered waves which have qualitatively prescribed profiles in the direction of propagation and are periodic in the transverse direction; in particular, they found three-dimensional waves whose profiles in the direction of propagation resemble periodic, quasiperiodic or generalised solitary waves. Three-dimensional waves which are periodic in the direction of propagation and have qualitatively prescribed profiles in the perpendicular direction have been studied by Groves [10] and Haragus and Kirchgässner [13], who found waves whose profiles in the transverse direction resemble periodic, quasiperiodic, solitary or generalised solitary waves. These results are special, 


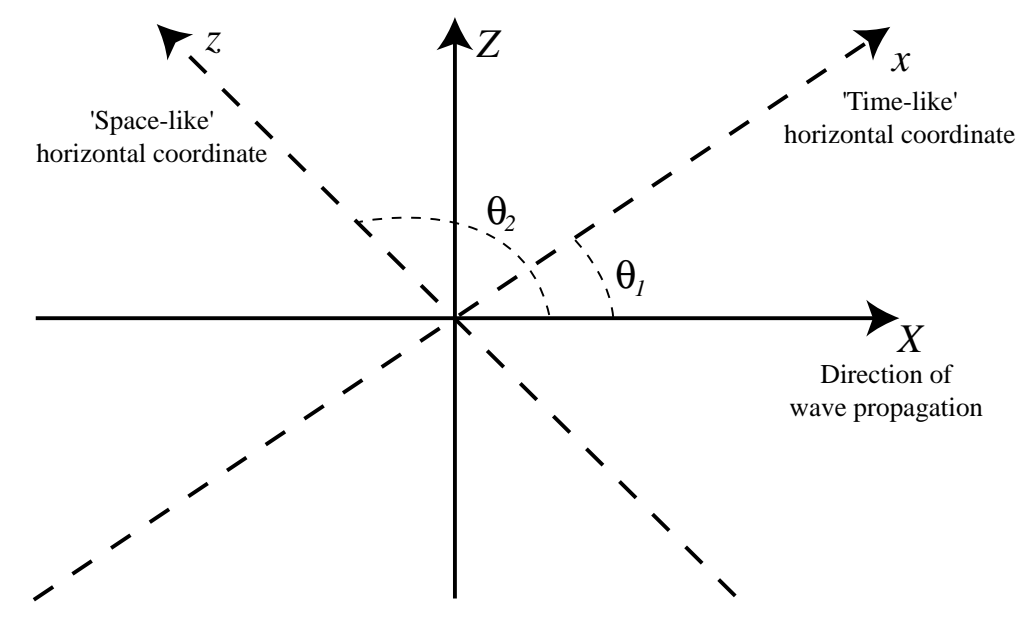

Figure 1: The waves have a qualitatively prescribed profile in the $x$ direction and are periodic in the $z$ direction; mathematically speaking these directions are respectively unbounded ('timelike') and bounded ('space like').

non-generic cases $\left(\theta_{1}=0, \theta_{2}= \pm \pi / 2\right.$ or $\left.\theta_{1}= \pm \pi / 2, \theta_{2}=0\right)$ of the waves discussed in the present paper which require a specific analysis.

The theory in this paper is based upon Kirchgässner's suggestion [19] that certain timeindependent problems can be studied by formulating them as evolutionary problems in which an unbounded spatial direction takes the role of the time-like variable. This idea has become known as 'spatial dynamics' and has been applied with success to a wide range of problems in applied mathematics. Kirchgässner himself [20] showed how to apply the method to the twodimensional travelling water-wave problem by writing the governing equations as an ill-posed quasilinear evolutionary system in which the unbounded spatial coordinate $\xi=X-c t$ plays the role of time. This formulation of the hydrodynamic problem can be studied using the centremanifold reduction theorem of Mielke [24], which states that the evolutionary system is locally equivalent to a system of ordinary differential equations whose solution set can, in theory, be analysed. Kirchgässner's method is the basis for several existence theories for two-dimensional travelling gravity-capillary water waves (see Iooss \& Kirchgässner [15, 16], Buffoni, Groves \& Toland [4], Buffoni \& Groves [3], Lombardi [21], Dias \& Iooss [7] and the references therein).

In this paper we apply Kirchgässner's method to the three-dimensional travelling gravitycapillary water-wave problem. Clearly any horizontal direction qualifies as an unbounded spatial coordinate, and with complete generality we choose the horizontal direction $x$ making an angle $\theta_{1}$ with the $X$-axis. To formulate the hydrodynamic equations as an evolutionary system in which $x$ is the time-like variable we exploit the observation by Luke [23] that the hydrodynamic problem follows from a variational principle. We treat the variational functional as an action functional in which a density is integrated over the $x$ direction and perform a Legendre transform to derive a formulation of the hydrodynamic problem as an infinite-dimensional Hamiltonian system in which $x$ is the time-like variable. Although this procedure is formal it delivers a candidate for a formulation of the hydrodynamic problem as an evolutionary system whose mathematical correctness is readily confirmed a posteriori; full details are given in Section 2.2. Mielke's reduction result specifies conditions on the spectrum of the linear operator in the evolutionary equation which essentially require that there are no further 'time-like' (unbounded) spatial directions; we 
therefore restrict our attention to waves which are periodic in a second horizontal direction $z$ which makes an angle $\theta_{2}$ with the $X$-axis. Notice that this restriction excludes in particular the 'fully localised' solitary waves which decay to zero in all horizontal directions. The existence of such solutions for the Euler equations remains an open problem, although they are known to exist in the long-wave approximation as solutions of generalised Kadomtsev-Petviashvili equations (see de Bouard \& Saut [6]).

The Hamiltonian structure of the evolutionary system plays a central role in the existence theories in this article. Mielke [25] has shown that the Kirchgässner reduction preserves Hamiltonian structure, so that the reduced system of equations is also a Hamiltonian system. In this fashion we show that the hydrodynamic problem is locally equivalent to a Hamiltonian system with a finite number of degrees of freedom, and our task is reduced to one of finding solutions of this finite-dimensional problem. Fortunately there is a cornucopia of existence theories for finitedimensional Hamiltonian systems, in particular for homoclinic and periodic solutions; the work lies in selecting a suitable existence result and verifying that our reduced Hamiltonian system satisfies its hypotheses.

Notice that there is a one-to-one correspondence between oblique waves in the $\left(x_{1}, z_{1}\right)$ and $\left(x_{2}, z_{2}\right)$ coordinate systems defined by the pairs of angles $\left(\theta_{1}^{1}, \theta_{2}\right)$ and $\left(\theta_{1}^{2}, \theta_{2}\right)$, so that

$$
x_{2}=\csc \left(\theta_{2}-\theta_{1}^{2}\right) \sin \left(\theta_{2}-\theta_{1}^{1}\right) x_{1}, \quad z_{2}=\csc \left(\theta_{1}^{1}-\theta_{2}\right) \sin \left(\theta_{1}^{2}-\theta_{1}^{1}\right) x_{1}+z_{1}:
$$

a simple calculation shows that a wave which has a certain profile in the $x_{1}$ direction and is $2 \pi / \nu$-periodic in $z_{1}$ corresponds to a wave which has a specific profile in the $x_{2}$ direction and is $2 \pi / \nu$-periodic in $z_{2}$. In principle one can therefore fix the value of the angle $\theta_{1}$ provided a full set of oblique waves for this choice of $\theta_{1}$ (that is a set of waves with all possible profiles in the $x$ direction) can be determined. This approach can, however, lead to more difficult existence proofs. For example, a wave which is doubly periodic in the $\left(x_{1}, z_{1}\right)$ coordinate system is typically not doubly periodic in the $\left(x_{2}, z_{2}\right)$ coordinate system, being periodic in $z_{2}$ and aperiodic in $x_{2}$. To find this wave it is necessary to find a periodic solution to the reduced equations on the centre manifold with $x_{1}$ as time or an aperiodic solution to the reduced equations on the centre manifold with $x_{2}$ as time; the former task is typically easier than the latter. In our analysis we take advantage of this extra flexibility gained by varying the angle $\theta_{1}$ and concentrate on certain types of profiles in the $x$ direction (periodic, solitary-wave or generalised solitary-wave profiles).

Oblique line waves are waves which are constant in the $z$-direction. We adopt this terminology since such a wave depends only upon one spatial direction $x$, which is however not aligned with the direction $X$ of wave propagation. A travelling wave moving with velocity $\mathbf{c}=c \mathbf{e}_{X}=c \sin \theta_{2} \mathbf{e}_{x}+c \sin \theta_{1} \mathbf{e}_{z}$ which is constant in the $z$ direction is clearly equivalent to a travelling wave moving with velocity $c \sin \theta_{2} \mathbf{e}_{x}$ which is constant in the $z$ direction, and the physical problem for the latter wave is independent of the value of $\theta_{1}$. Indeed, an examination of the Euler equations for such waves shows that they are related to the Euler equations for classical two-dimensional travelling waves ( $z$-independent waves with $\theta_{1}=0$ and $\theta_{2}=\pi / 2$ in the present terminology) with speed $c \sin \theta_{2}$ via a simple scaling (see the remarks near the end of Section 2.1). It follows that the set of oblique line waves, for any choices of $\theta_{1}, \theta_{2}$ and $c$, lies in oneto-one correspondence with the set of two-dimensional travelling waves. We therefore confine ourselves here to a description of some of the oblique line waves which can be obtained from the extensive literature on two-dimensional travelling waves together with some results which are also novel in the two-dimensional context (see below). 
The hydrodynamic problem depends upon the angles $\theta_{1}$ and $\theta_{2}$, the period $\nu$ in the $z$ direction and the dimensionless physical parameters $\alpha=g h / c^{2}$ and $\beta=T / h c^{2}$ where $g, h, c$ and $T$ denote respectively the acceleration due to gravity, the depth of the fluid in its undisturbed state, the speed of the travelling wave and the coefficient of surface tension. We study bifurcation phenomena by fixing values of $\alpha, \beta, \theta_{1}, \theta_{2}, \nu$ and perturbing around them with a bifurcation parameter $\lambda \in \mathbb{R}^{5}$; the dimension of the reduced Hamiltonian system is equal to the number of purely imaginary eigenvalues at criticality and the reduced equations are valid for values of the bifurcation parameter in a neighbourhood of zero. The reduction procedure is explained in detail in Section 2.3.

A purely imaginary eigenvalue $i \kappa$ with corresponding eigenvector in the $n$th Fourier mode corresponds to a linear travelling water wave of the form $\eta(X, Z)=\eta_{\kappa, n} e^{\mathrm{i} k X+\mathrm{i} \ell Z}$ with

$$
k=\sin \theta_{2} \kappa+\sin \theta_{1} n \nu, \quad \ell=-\cos \theta_{2} \kappa-\cos \theta_{1} n \nu,
$$

and it is well known that a solution of this kind exists if and only if $k$ and $\ell$ satisfy the classical dispersion relation

$$
\mathcal{D}(k, \ell)=-k^{2}+\left(\alpha+\beta\left(k^{2}+\ell^{2}\right)\right) \sqrt{k^{2}+\ell^{2}} \tanh \sqrt{k^{2}+\ell^{2}}=0 .
$$

The above observation has an elegant geometric interpretation: purely imaginary eigenvalues $i \kappa$ correspond to intersections in the $(k, \ell)$-plane of the real branches $C_{\mathrm{dr}}$ of the dispersion relation with the parallel lines $K_{n}, n=0, \pm 1, \pm 2, \ldots$ given in parametric form by

$$
K_{n}=\left\{(k, \ell) \in \mathbb{R}^{2}: k=\sin \theta_{2} \kappa+\sin \theta_{1} n \nu, \ell=-\cos \theta_{2} \kappa-\cos \theta_{1} n \nu, \kappa \in \mathbb{R}\right\}
$$

(see Figure 2(b)). A point of intersection of $K_{n}$ and $C_{\mathrm{dr}}$ corresponds to a purely imaginary mode $n$ eigenvalue $i \kappa$; its imaginary part is the value of the parameter $\kappa$ at the point of intersection, that is the value of $K_{0}$ in the $\left(K_{0}, L\right)$-coordinate system at the intersection, where

$$
L=\left\{(k, \ell) \in \mathbb{R}^{2}: k=\sin \theta_{1} \mu, \ell=-\cos \theta_{1} \mu, \mu \in \mathbb{R}\right\} .
$$

The geometric multiplicity of the eigenvalue i $\kappa$ is given by the number of distinct lines in the family $\left\{K_{n}\right\}$ which intersect $C_{\mathrm{dr}}$ at this parameter value, and a tangent intersection between $K_{n}$ and $C_{\mathrm{dr}}$ indicates that each eigenvector in mode $n$ has an associated Jordan chain of length 2.

The $(\beta, \alpha)$ parameter plane is divided into regions I, II and III in which $C_{\mathrm{dr}}$ has respectively zero, one and two nontrivial bounded branches (see Figure 2(a)). These regions are delineated by the line $\{\alpha=1\}$ and the curve

$$
\Gamma=\left\{(\beta, \alpha)=\left(-\frac{1}{2 \sinh ^{2} \kappa}+\frac{1}{2 \kappa \tanh \kappa}, \frac{\kappa^{2}}{2 \sinh ^{2} \kappa}+\frac{\kappa}{2 \tanh \kappa}\right): \kappa \in[0, \infty)\right\},
$$

which are also associated with bifurcation phenomena in the Kirchgässner reduction for twodimensional travelling gravity-capillary water waves [20]. Regions II and III are in fact each divided into two subregions, at the mutual boundary of which the qualitative shape of the branches changes, namely from convex to nonconvex. The dimension of the central subspace is given by the number of intersections of a discrete family of parallel lines with a bounded set of curves and is therefore always finite (see Figure 2(b)). Notice, however, that in the case of gravity waves 

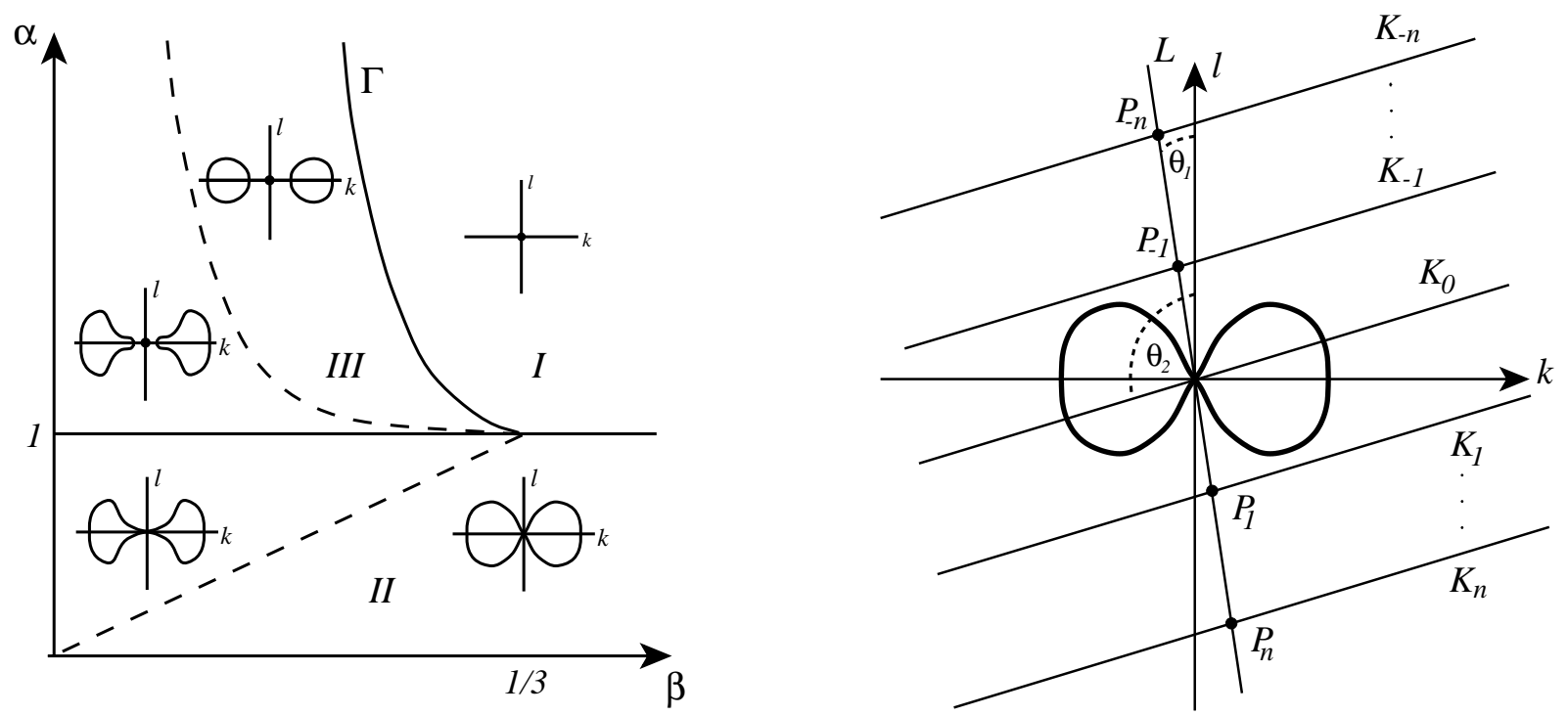

Figure 2: (a) Shape of the real branches of the dispersion relation $\mathcal{D}(k, \ell)=0$. (b) Position of the lines $K_{n}$ in the $(k, \ell)$-plane; the lines $K_{0}$ and $L$ form respectively angles $\theta_{2}$ and $\theta_{1}$ with the positive $\ell$-axis.

$(\beta=0)$ the set $C_{\mathrm{dr}}$ is always unbounded, so that the linear operator always has infinitely many purely imaginary eigenvalues. Existence theories for periodic gravity waves are therefore likely to encounter small-divisor problems. This observation has already been made in a short note by Plotnikov [28], and although Plotnikov gave a sketch of an existence proof for doubly periodic travelling gravity waves using superconvergence methods this problem remains essentially open. Pego \& Quintero [27] have recently constructed an infinite-dimensional centre manifold for a semilinear model equation (the steady-wave Benney-Luke equation) with an infinite-dimensional centre subspace, but their method does not apply to a spatial dynamics formulation for travelling water waves, which is a quasilinear problem.

A catalogue of bifurcation scenarios in which the number of purely imaginary eigenvalues changes upon varying a parameter is presented in Section 3. We begin with bifurcation scenarios involving only mode 0 eigenvalues (Section 3.2); these situations correspond to bifurcation phenomena involving oblique line waves. Oblique line waves always lie in an invariant subspace of the reduced system which do not depend upon the parameters $\nu$ and $\theta_{1}$. These two parameters play no role in the oblique line-wave problem. Purely imaginary eigenvalues correspond to intersections of the line $K_{0}$, which depends upon the angle $\theta_{2}$, with the curve $C_{\mathrm{dr}}$, which depends upon the physical parameters $\alpha, \beta$, and bifurcation diagrams are obtained by fixing the physical parameters $\alpha, \beta$ and varying the angle $\theta_{2}$ (see Figure 4) or vice versa (see Figure 5). The qualitative difference in the geometric shape of $C_{\mathrm{dr}}$ in the two subregions of region II or II plays no role in oblique line-wave bifurcation theory but leads to a wealth of new bifurcation phenomena in bifurcation scenarios involving higher Fourier modes.

Bifurcation scenarios involving higher Fourier modes are readily detected by fixing the values of the physical parameters $(\beta, \alpha)$ (in region II or III) and the angles $\theta_{1}, \theta_{2}$ and varying the 
frequency $\nu$ : by decreasing a large initial value of $\nu$ one finds a sequence of critical frequencies

$$
\nu=\nu_{\mathrm{c}}, \frac{\nu_{\mathrm{c}}}{2}, \frac{\nu_{\mathrm{c}}}{3}, \ldots,
$$

at which the pairs of lines $\left(K_{1}, K_{-1}\right),\left(K_{2}, K_{-2}\right),\left(K_{3}, K_{-3}\right), \ldots$ touch the dispersion curve $C_{\mathrm{dr}}$ in the $(k, \ell)$-plane for the first time. We find that there are three basic bifurcation scenarios at $\nu=\nu_{\mathrm{c}}$, when the lines $K_{1}$ and $K_{-1}$ are tangent to $C_{\mathrm{dr}}$ in the $(k, \ell)$-plane (Figure 6) and a plus-minus pair of geometrically simple purely imaginary eigenvalues, each with a Jordan chain of length 2, are created; the cases are distinguished according to whether the line $K_{0}$ intersects $C_{\mathrm{dr}}$ in zero, two or four points. These bifurcation scenarios are known as the Hamiltonian-Hopf bifurcation, the Hamiltonian $\left(\mathrm{i} \omega_{0}\right)^{2} \mathrm{i} \omega_{1}$ resonance and the Hamiltonian $\left(\mathrm{i} \omega_{0}\right)^{2} \mathrm{i} \omega_{1} \mathrm{i} \omega_{2}$ resonance. Notice that the value of $\theta_{1}$ does not influence the nature of the bifurcations, it only determines the relative position of the eigenvalues on the imaginary axis at bifurcation points. In particular, there are special values of $\theta_{1}$ for which purely imaginary eigenvalues vanish or have the same value as purely imaginary eigenvalues in different Fourier modes; degenerate cases of the standard bifurcations indicated above arise in this fashion (see Figure 7). In addition there are values of $\alpha, \beta$ for which $K_{1}$ and $K_{-1}$ are tangent to $C_{\mathrm{dr}}$ at two points each because of the nonconvexity of $C_{\mathrm{dr}}$; Figure 8 illustrates several bifurcation scenarios involving double tangencies of this kind.

The solution set of the reduced Hamiltonian system is examined for a representative sample of the above bifurcation scenarios in Section 4, beginning with periodic solutions in Section 4.2. A $2 \pi / \kappa$-periodic solution of the reduced Hamiltonian system corresponds to a doubly periodic travelling water wave which is $2 \pi / \kappa$-periodic in $x$ and $2 \pi / \nu$-periodic (or constant if only mode 0 eigenvalues are considered) in $z$, and two representative waves of this kind are sketched in Figure 3. Such waves may be found by applying an appropriate version of the Lyapunov centre theorem. This programme is carried out for oblique line periodic waves and hence also for twodimensional travelling waves. We show that there is one Lyapunov family in region II and two in region III with the exception of those points on a family of curves $\left\{R_{k}\right\}_{k=2}^{\infty}$ where a Hamiltonian $-1: k$ eigenvalue resonance takes place. (In a Hamiltonian $-1: k$ or $1:-k$ resonance two pairs $\pm \mathrm{i} \omega, \pm \mathrm{i} k \omega$ of eigenvalues with opposite Krein signatures lie on the imaginary axis; the sign indicates which pair has negative Krein signature.) Two-degree-of-freedom Hamiltonian systems with two pairs of imaginary eigenvalues in $1: k$ or $1:-k$ resonance were examined by Schmidt [30] and Duistermaat [9], who presented an existence theory based upon persistence results for periodic solutions of the fourth-order Birkhoff normal form associated with the resonance; we apply their theory to resolve the bifurcation picture at the curves $R_{k}$. We also discuss doubly periodic waves associated with mode 0,1 and -1 eigenvalues. In particular we give a result obtained via the classical nonresonant Lyapunov centre theorem guaranteeing the existence of doubly periodic waves with any prescribed fundamental domain together with a result obtained via the Lyapunov centre theorem for Hamiltonians with positive definite quadratic parts formulated by Weinstein [32] and Moser [26].

In Section 4.3 we consider waves which are not periodic in the $x$ direction, discussing waves which are constant in the $z$ direction and waves associated with mode 0,1 and -1 eigenvalues. In the former class we confine ourselves to stating some results which are obtained as a consequence of the corresponding results for two-dimensional travelling waves, namely bifurcations connected with the Hamiltonian $0^{2}$ resonance and the Hamiltonian-Hopf bifurcation; we obtain travelling waves which have the profile of a solitary wave in the $x$ direction. In the second class 

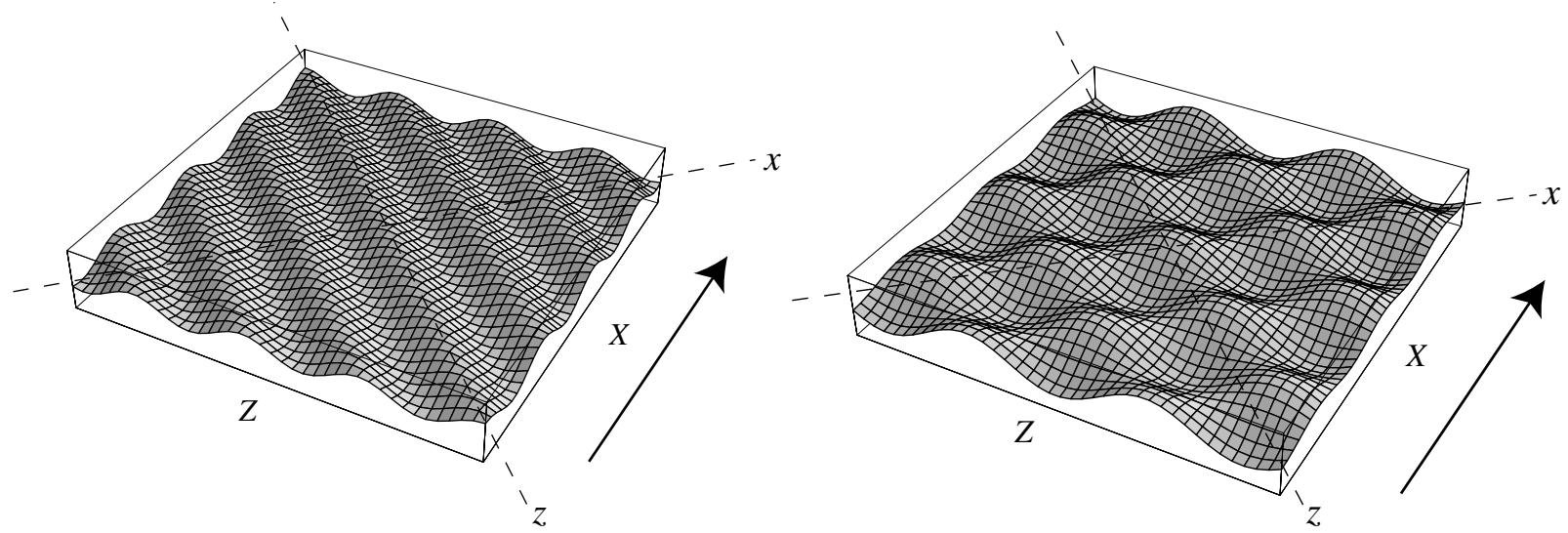

Figure 3: The wave on the left is periodic in $x$ and independent of $z$ while the wave on the right is periodic in $x$ and $z$; they both move with constant speed and without change of shape in the $X$ direction (arrowed).

we treat waves connected with the Hamiltonian-Hopf bifurcation and the Hamiltonian $\left(\mathrm{i} \omega_{0}\right)^{2} \mathrm{i} \omega_{1}$ resonance; the theory is based upon persistence arguments for solutions of finite-order Birkhoff normal forms and yields travelling waves which are periodic in $z$ and have the profile of respectively a solitary wave and a generalised solitary wave (a solitary wave with periodic ripples far up- and downstream) in the $x$ direction. We make particular use of results by Iooss \& Pérouème [17] and Buffoni \& Groves [3] for the Hamiltonian-Hopf bifurcation and by Groves \& Mielke [11] and Lombardi [22] for the Hamiltonian $\left(\mathrm{i} \omega_{0}\right)^{2} \mathrm{i} \omega_{1}$ resonance.

The above observations show that the hydrodynamic problem in its spatial dynamics formulation exhibits a wealth of bifurcation phenomena even when only mode 0,1 and -1 eigenvalues are considered. Working through mode $\pm 2, \pm 3, \ldots$ eigenvalues, one can in principle systematically compile a complete catalogue of all the bifurcation scenarios embedded in this problem, and taking all parameters into account, it is possible to detect virtually any bifurcation or resonance known in Hamiltonian systems theory. In this sense one can regard the present version of the water-wave problem as a paradigm for finite-dimensional Hamiltonian systems and conservative pattern-formation problems.

Acknowledgement We would like to thank Prof. A. Mielke for suggesting this problem to us during the Steady Water Waves session at the Equadiff conference in Berlin, August 1999.

\section{The mathematical problem}

\subsection{The Euler equations for oblique water waves}

The physical problem in question concerns the three-dimensional flow of a perfect fluid of unit density subject to the forces of gravity and surface tension. Let $(X, Y, Z)$ denote the usual Cartesian coordinates. The fluid occupies the domain $D_{\eta}=\{(X, Y, Z): X, Z \in \mathbb{R}, Y \in$ $(0, h+\eta(X, Z, t))\}$, where $\eta>-h$ is a function of the spatial coordinates $X, Z$ and of time $t$, and $h$ represents the depth of the fluid in its undisturbed state. In terms of an Eulerian velocity 
potential $\phi$, the mathematical problem is to solve Laplace's equation

$$
\phi_{X X}+\phi_{Y Y}+\phi_{Z Z}=0 \quad \text { in } D_{\eta}
$$

with kinematic boundary conditions

$$
\begin{aligned}
& \phi_{Y}=0 \\
& \text { on } Y=0 \text {, } \\
& \phi_{Y}=\eta_{t}+\eta_{X} \phi_{X}+\eta_{Z} \phi_{Z} \\
& \text { on } Y=h+\eta
\end{aligned}
$$

that the water cannot permeate the rigid bottom at $Y=0$ or the free surface at $Y=h+\eta(X, Z, t)$ and the dynamic boundary condition

$$
\begin{aligned}
\phi_{t}= & -\frac{1}{2}\left(\phi_{X}^{2}+\phi_{Y}^{2}+\phi_{Z}^{2}\right)-g \eta \\
& +\sigma\left[\frac{\eta_{X}}{\sqrt{1+\eta_{X}^{2}+\eta_{Z}^{2}}}\right]_{X}+\sigma\left[\frac{\eta_{Z}}{\sqrt{1+\eta_{X}^{2}+\eta_{Z}^{2}}}\right]_{Z}+B \quad \text { on } Y=h+\eta
\end{aligned}
$$

at the free surface. Here $g$ is the acceleration due to gravity, $\sigma$ is the coefficient of surface tension and $B$ is a constant called the Bernoulli constant (e.g. see Stoker [31, $\S 11,2.1]$ ).

Travelling waves are water waves which are uniformly translating in the $X$ direction (there is no loss of generality in choosing a specific horizontal direction since the hydrodynamic problem (1)-(4) is invariant under rotations of the $(X, Z)$-plane). These waves are described by solutions of (1)-(4) of the special form $\eta(X, Z, t)=\eta(X-c t, Z), \phi(Z, Y, Z, t)=\phi(X-c t, Y, Z)$. Substituting this form of $\eta, \phi$ into (1)-(4) and introducing the dimensionless independent variables

$$
\left(X^{\prime}, Y^{\prime}, Z^{\prime}\right)=\frac{1}{h}(X, Y, Z)
$$

and dependent variables

$$
\eta^{\prime}\left(X^{\prime}, Z^{\prime}\right)=\frac{1}{h} \eta(X, Z), \quad \phi^{\prime}\left(X^{\prime}, Y^{\prime}, Z^{\prime}\right)=\frac{1}{c h} \phi(X, Y, Z),
$$

one obtains the equations

$$
\begin{array}{ll}
\phi_{X X}+\phi_{Y Y}+\phi_{Z Z}=0, & 0<Y<1+\eta, \\
\phi_{Y}=0 & \text { on } Y=0, \\
\phi_{Y}=\eta_{X} \phi_{X}+\eta_{Z} \phi_{Z}-\eta_{X} & \text { on } Y=1+\eta
\end{array}
$$

and

$$
\begin{aligned}
-\phi_{X} & +\frac{1}{2}\left(\phi_{X}^{2}+\phi_{Y}^{2}+\phi_{Z}^{2}\right)-\alpha \eta \\
& -\beta\left[\frac{\eta_{X}}{\sqrt{1+\eta_{X}^{2}+\eta_{Z}^{2}}}\right]_{X}-\beta\left[\frac{\eta_{Z}}{\sqrt{1+\eta_{X}^{2}+\eta_{Z}^{2}}}\right]_{Z}=0 \quad \text { on } Y=1+\eta,
\end{aligned}
$$

in which the primes have been dropped and $X$ is now a shorthand for the variable $X-c t$ and the Bernoulli constant has been set to zero. The dimensionless numbers

$$
\alpha=g h / c^{2}, \quad \beta=\sigma / h c^{2}
$$


are respectively the inverse square of the Froude number and the Weber number. Observe that the hydrodynamic problem (5)-(8) has two discrete symmetries: it is invariant under the transformations

$$
\begin{array}{lll}
X \mapsto X, \quad Z \mapsto-Z, & \eta \mapsto \eta, \quad \Phi \mapsto \Phi, \\
X \mapsto-X, \quad Z \mapsto Z, \quad \eta \mapsto \eta, \quad \Phi \mapsto-\Phi,
\end{array}
$$

which arise as a remnant of the rotational invariance of the original water-wave equations (1)-(4).

This paper treats waves which are periodic in a distinguished horizontal direction $z$ and have a qualitatively prescribed profile in another distinguished horizontal direction $x$. The $x$ - and $z$ axes make different angles $\theta_{1}, \theta_{2}$ with the $X$-axis, where $\theta_{1}, \theta_{2} \in(-\pi, \pi)$ are chosen arbitrarily (see Figure 1). We therefore seek solutions of (5)-(8) of the form

$$
\eta(X, Z)=\tilde{\eta}(\tilde{x}, \tilde{z}), \quad \phi(X, Y, Z)=\tilde{\phi}(\tilde{x}, Y, \tilde{z}),
$$

where

$$
\tilde{x}=\sin \theta_{2} X-\cos \theta_{2} Z, \quad \tilde{z}=\sin \theta_{1} X-\cos \theta_{1} Z
$$

and $\eta, \phi$ are $P$-periodic in $z$. These solutions satisfy the equations

$$
\begin{aligned}
& \phi_{x x}+\phi_{Y Y}+\nu^{2} \phi_{z z}+2 \nu \cos \left(\theta_{1}-\theta_{2}\right) \phi_{x z}=0, \\
& \phi_{Y}=0 \\
& \phi_{Y}=-\sin \theta_{2} \eta_{x}-\nu \sin \theta_{1} \eta_{z}+\eta_{x} \phi_{x}+\nu^{2} \eta_{z} \phi_{z} \\
& +\nu \cos \left(\theta_{1}-\theta_{2}\right)\left(\eta_{x} \phi_{z}+\eta_{z} \phi_{x}\right) \\
& -\sin \theta_{2} \phi_{x}-\nu \sin \theta_{1} \phi_{z}+\frac{1}{2}\left(\phi_{x}^{2}+\phi_{Y}^{2}+\nu^{2} \phi_{z}^{2}+2 \nu \cos \left(\theta_{1}-\theta_{2}\right) \phi_{x} \phi_{z}\right)+\alpha \eta \\
& -\beta\left(\frac{\eta_{x}}{R}\right)_{x}-\beta \nu^{2}\left(\frac{\eta_{z}}{R}\right)_{z}-\beta \nu \cos \left(\theta_{1}-\theta_{2}\right)\left[\left(\frac{\eta_{x}}{R}\right)_{z}+\left(\frac{\eta_{z}}{R}\right)_{x}\right]=0 \quad \text { on } Y=1+\eta,
\end{aligned}
$$

in which the tildes have been dropped, the period in $z$ has been normalized to $2 \pi$ and $\nu=2 \pi / P$,

$$
R=\sqrt{1+\eta_{x}^{2}+\nu^{2} \eta_{z}^{2}+2 \nu \cos \left(\theta_{1}-\theta_{2}\right) \eta_{x} \eta_{z}} .
$$

Apart from certain special values of the angles $\theta_{1}, \theta_{2}$, the equations (12)-(15) for oblique waves inherit only one of the symmetries of (5)-(8), namely the composition of (9) and (10), so that it is invariant under the transformation

$$
X \mapsto-X, \quad Z \mapsto-Z, \quad \eta \mapsto \eta, \quad \Phi \mapsto-\Phi .
$$

These special angular values are $\theta_{1}=0, \theta_{2}= \pm \pi / 2$ and $\theta_{1}= \pm \pi / 2, \theta_{2}=0$, for which (12)-(15) are invariant under both (9) and (10).

Oblique line waves are solutions of equation (12)-(15) above which do not depend upon $z$. Observe that oblique line-wave solutions are transformed into solutions of the mathematical problem for two-dimensional travelling waves ( $z$-independent solutions of (12)-(15) with $\theta_{1}=$ $\left.0, \theta_{2}=\pi / 2\right)$ by the transformation $(\phi, \eta) \mapsto\left(\sin \theta_{2} \phi, \eta\right),(\beta, \alpha) \mapsto\left(\beta \sin ^{2} \theta_{2}, \alpha \sin ^{2} \theta_{2}\right)$. This observation will be exploited at several points in the subsequent analysis.

Equations (12)-(15) depend upon two independent horizontal directions $x$ and $z$ which are respectively unbounded and bounded and the bounded vertical direction $y$. In Section 2.2 below we formulate this hydrodynamic problem as an evolutionary problem in which the unbounded direction $x$ plays the role of time and the bounded directions $y$ and $z$ are treated as spatial coordinates. 


\subsection{Hamiltonian formulation}

Observe that equations (12)-(15) follow from the formal variational principle

$$
\begin{gathered}
\delta \iint_{0}^{2 \pi}\left\{\int_{0}^{1+\eta}\left(-\sin \theta_{2} \phi_{x}-\nu \sin \theta_{1} \phi_{z}+\frac{1}{2}\left(\phi_{x}^{2}+\phi_{Y}^{2}+\nu^{2} \phi_{z}^{2}+2 \nu \cos \left(\theta_{1}-\theta_{2}\right) \phi_{x} \phi_{z}\right)\right) \mathrm{d} Y\right. \\
\left.\quad+\frac{1}{2} \alpha \eta^{2}+\beta(R-1)\right\} \mathrm{d} z \mathrm{~d} x=0
\end{gathered}
$$

in which the variation is taken in $(\eta, \phi)$. The change of variable

$$
\phi(x, Y, z)=\Phi(x, y, z), \quad Y=y(1+\eta(x, z))
$$

maps the variable domain $\{(x, Y, z): x \in \mathbb{R}, z \in(0,2 \pi), 0<Y<1+\eta(x, z)\}$ to $\mathbb{R} \times$ $(0,2 \pi) \times(0,1)$ and replaces $\phi$ with a function $\Phi$ defined on this fixed domain. The variational principle (17) is transformed into

$$
\delta \mathcal{L}=0, \quad \mathcal{L}=\int L\left(\eta, \Phi, \eta_{x}, \Phi_{x}\right) \mathrm{d} x,
$$

where

$$
\begin{aligned}
& L\left(\eta, \Phi, \eta_{x}, \Phi_{x}\right)= \\
& \qquad \int_{\Sigma}\left\{-\sin \theta_{2}\left(\Phi_{x}-\frac{y \eta_{x}}{1+\eta} \Phi_{y}\right)-\nu \sin \theta_{1}\left(\Phi_{z}-\frac{y \eta_{z}}{1+\eta} \Phi_{y}\right)\right. \\
& \quad+\frac{1}{2}\left(\Phi_{x}-\frac{y \eta_{x}}{1+\eta} \Phi_{y}\right)^{2}+\frac{\Phi_{y}^{2}}{2(1+\eta)^{2}}+\frac{1}{2} \nu\left(\Phi_{z}-\frac{y \eta_{z}}{1+\eta} \Phi_{y}\right)^{2} \\
& \left.\quad+\nu \cos \left(\theta_{1}-\theta_{2}\right)\left(\Phi_{x}-\frac{y \eta_{x}}{1+\eta} \Phi_{y}\right)\left(\Phi_{z}-\frac{y \eta_{z}}{1+\eta} \Phi_{y}\right)\right\}(1+\eta) \mathrm{d} y \mathrm{~d} z \\
& \quad+\int_{S}\left\{\frac{1}{2} \alpha \eta^{2}+\beta(R-1)\right\} \mathrm{d} z,
\end{aligned}
$$

and $\Sigma=(0,1) \times(0,2 \pi), S=(0,2 \pi)$.

The next step is to carry out a Legendre transform. Define new variables $\omega$ and $\xi$ by the formulae

$$
\begin{aligned}
\omega= & \frac{\delta L}{\delta \eta_{x}} \\
= & \int_{0}^{1}\left\{\sin \theta_{2}-\left(\Phi_{x}-\frac{y \eta_{x}}{1+\eta} \Phi_{y}\right)-\nu \cos \left(\theta_{1}-\theta_{2}\right)\left(\Phi_{z}-\frac{y \eta_{z}}{1+\eta} \Phi_{y}\right)\right\} y \Phi_{y} \mathrm{~d} y, \\
& +\frac{\beta}{R}\left(\eta_{x}+\nu \cos \left(\theta_{1}-\theta_{2}\right) \eta_{z}\right), \\
\xi= & \frac{\delta L}{\delta \Phi_{x}} \\
= & \left(-\sin \theta_{2}+\left(\Phi_{x}-\frac{y \eta_{x}}{1+\eta} \Phi_{y}\right)+\nu \cos \left(\theta_{1}-\theta_{2}\right)\left(\Phi_{z}-\frac{y \eta_{z}}{1+\eta} \Phi_{y}\right)\right)(1+\eta),
\end{aligned}
$$


in which the variational derivatives are taken formally in respectively $L^{2}(S)$ and $L^{2}(\Sigma)$, and define the Hamiltonian function by

$$
\begin{aligned}
& H(\eta, \omega, \Phi, \xi) \\
& =\int_{\Sigma} \xi \Phi_{x} \mathrm{~d} y \mathrm{~d} z+\int_{S} \omega \eta_{x} \mathrm{~d} z-L\left(\eta, \Phi, \eta_{x}, \Phi_{x}\right) \\
& =\int_{\Sigma}\left\{\sin \theta_{2} \xi+\nu \sin \theta_{1}(1+\eta)\left(\Phi_{z}-\frac{y \eta_{z}}{1+\eta} \Phi_{y}\right)\right. \\
& \quad+\frac{\xi^{2}}{2(1+\eta)}-\frac{\Phi_{y}^{2}}{2(1+\eta)}-\frac{1}{2} \nu^{2} \sin ^{2}\left(\theta_{1}-\theta_{2}\right)(1+\eta)\left(\Phi_{z}-\frac{y \eta_{z}}{1+\eta} \Phi_{y}\right)^{2} \\
& \left.\quad-\nu \cos \left(\theta_{1}-\theta_{2}\right)\left(\xi+(1+\eta) \sin \theta_{2}\right)\left(\Phi_{z}-\frac{y \eta_{z}}{1+\eta} \Phi_{y}\right)\right\} \mathrm{d} y \mathrm{~d} z \\
& +\int_{S}\left\{-\frac{1}{2} \alpha \eta^{2}-\nu \cos \left(\theta_{1}-\theta_{2}\right) W \eta_{z}+\frac{1}{2} \sin ^{2} \theta_{2}(1+\eta)\right. \\
& \left.\quad+\beta-\left(\beta^{2}-W^{2}\right)^{1 / 2}\left(1+\nu^{2} \sin ^{2}\left(\theta_{1}-\theta_{2}\right) \eta_{z}^{2}\right)^{1 / 2}\right\} \mathrm{d} z
\end{aligned}
$$

where

$$
W=\omega+\frac{1}{1+\eta} \int_{0}^{1} y \Phi_{y} \xi \mathrm{d} y .
$$

According to the above calculation the equations

$$
\eta_{x}=\frac{\delta H}{\delta \omega}, \quad \omega_{x}=-\frac{\delta H}{\delta \eta}, \quad \Phi_{x}=\frac{\delta H}{\delta \xi}, \quad \xi_{x}=-\frac{\delta H}{\delta \Phi}
$$

formally represent Hamilton's equations for a formulation of (12)-(15) as a Hamiltonian system. Writing down these equations, one finds that $(\eta, \omega, \Phi, \xi)=\left(0,0,0,-\sin \theta_{2}\right)$ is always a solution (the state of rest in the hydrodynamic problem); we therefore introduce the new variable $\Psi=$ $\xi+\sin \theta_{2}$ and obtain the Hamiltonian

$$
\begin{array}{r}
H(\eta, \omega, \Phi, \Psi) \\
=\int_{\Sigma}\left\{\sin \theta_{2} \Psi+\nu \sin \theta_{1}(1+\eta)\left(\Phi_{z}-\frac{y \eta_{z}}{1+\eta} \Phi_{y}\right)+\frac{\left(\Psi-\sin \theta_{2}\right)^{2}}{2(1+\eta)}\right. \\
\quad-\frac{\Phi_{y}^{2}}{2(1+\eta)}-\frac{1}{2} \nu^{2} \sin ^{2}\left(\theta_{1}-\theta_{2}\right)(1+\eta)\left(\Phi_{z}-\frac{y \eta_{z}}{1+\eta} \Phi_{y}\right)^{2} \\
\left.\quad-\nu \cos \left(\theta_{1}-\theta_{2}\right)\left(\Psi+\eta \sin \theta_{2}\right)\left(\Phi_{z}-\frac{y \eta_{z}}{1+\eta} \Phi_{y}\right)\right\} \mathrm{d} y \mathrm{~d} z \\
+\int_{S}\left\{-\frac{1}{2} \alpha \eta^{2}-\nu \cos \left(\theta_{1}-\theta_{2}\right) W \eta_{z}+\frac{1}{2} \sin ^{2} \theta_{2}(\eta-1)\right. \\
\left.\quad+\beta-\left(\beta^{2}-W^{2}\right)^{1 / 2}\left(1+\nu^{2} \sin ^{2}\left(\theta_{1}-\theta_{2}\right) \eta_{z}^{2}\right)^{1 / 2}\right\} \mathrm{d} z,
\end{array}
$$


in which

$$
W=\omega+\frac{1}{1+\eta} \int_{0}^{1} y \Phi_{y}\left(\Psi-\sin \theta_{2}\right) \mathrm{d} y .
$$

We introduce the Hilbert spaces

$$
\begin{aligned}
& H_{\mathrm{per}}^{t}(S)=\left\{u \in H_{\mathrm{loc}}^{t}(\mathbb{R}): u(x+2 \pi)=u(x), x \in \mathbb{R}\right\}, \\
& H_{\mathrm{per}}^{t}(\Sigma)=\left\{u \in H_{\mathrm{loc}}^{t}((0,1) \times \mathbb{R}): u(x+2 \pi, y)=u(x, y), x \in \mathbb{R}, y \in(0,1)\right\},
\end{aligned}
$$

and define

$$
X_{t}=H_{\text {per }}^{t+1}(S) \times H_{\text {per }}^{t}(S) \times H_{\text {per }}^{t+1}(\Sigma) \times H_{\text {per }}^{t}(\Sigma) .
$$

Take $s \in(0,1 / 2)$ and consider the symplectic manifold $(M, \Omega)$, where $M=X_{s}$ and $\Omega$ is the position-independent 2-form on $M$ given by

$$
\begin{aligned}
& \left.\Omega\right|_{m}\left(\left(\eta_{1}, \omega_{1}, \Phi_{1}, \Psi_{1}\right),\left(\eta_{2}, \omega_{2}, \Phi_{2}, \Psi_{2}\right)\right)= \\
& \int_{S}\left(\omega_{2} \eta_{1}-\eta_{2} \omega_{1}\right) \mathrm{d} z+\int_{\Sigma}\left(\Psi_{2} \Phi_{1}-\Phi_{2} \Psi_{1}\right) \mathrm{d} y \mathrm{~d} z
\end{aligned}
$$

(the canonical 2-form with respect to the $L^{2}(S) \times L^{2}(S) \times L^{2}(\Sigma) \times L^{2}(\Sigma)$-inner product). The set

$$
N=\left\{(\eta, \omega, \Phi, \Psi) \in X_{s+1}:|W(z)|<\beta, \eta(z)>-1, z \in[0,2 \pi]\right\},
$$

in which $W$ is defined by (19) is a manifold domain of $M$ : the pointwise constraints are valid since $\eta \in H_{\text {per }}^{s+1} \subset C(\bar{S})$ and it follows from the properties of the Sobolev spaces $X_{s}$ given in ref. [11, Appendix A] that the same is true of $W$. The results stated in ref. [11] also show that the function $H$ given by (18) belongs to $C^{\infty}(N, \mathbb{R})$ and that $\mathrm{d} H[n]:\left.N \rightarrow T^{*} N\right|_{n}$ extends to an operator $\left.\mathbf{d} H\right|_{n}:\left.N \rightarrow T^{*} M\right|_{n}$ for each $n \in N$. The triple $(M, \Omega, H)$ is therefore a Hamiltonian system.

It remains to compute Hamilton's equations and to confirm that a solution to them defines a solution of (12)-(15). Denote the Hamiltonian vector field associated with $(M, \Omega, H)$ by $v_{H}$. Recall that the point $n \in N$ belongs to $\mathcal{D}\left(v_{H}\right)$ with $\left.v_{H}\right|_{n}=\left.\bar{v}\right|_{n}$ if and only if

$$
\left.\Omega\right|_{n}\left(\left.\bar{v}\right|_{n},\left.v_{1}\right|_{n}\right)=\left.\mathbf{d} H\right|_{n}\left(\left.v_{1}\right|_{n}\right)
$$

for all tangent vectors $\left.\left.v_{1}\right|_{n} \in T M\right|_{n}$. Using this criterion one finds that $\mathcal{D}\left(v_{H}\right)$ is the set of functions $(\eta, \omega, \Phi, \Psi) \in N$ that satisfy the boundary conditions

$$
\begin{aligned}
& \Phi_{y}=0 \\
& -\frac{\Phi_{y}}{1+\eta}-\nu \sin \theta_{1} \eta_{z}+\nu \sin \theta_{2} \cos \left(\theta_{1}-\theta_{2}\right) \eta_{z}+\nu^{2} \sin ^{2}\left(\theta_{1}-\theta_{2}\right)\left(\Phi_{z}-\frac{\eta_{z} \Phi_{y}}{1+\eta}\right) \eta_{z} \Phi_{y} \\
& \quad+\left(\Psi-\sin \theta_{2}\right) \frac{W}{1+\eta}\left(\frac{1+\nu^{2} \sin ^{2}\left(\theta_{1}-\theta_{2}\right) \eta_{z}^{2}}{\beta^{2}-W^{2}}\right)^{1 / 2}=0 \quad \text { on } y=1
\end{aligned}
$$

and that Hamilton's equations

$$
u_{x}=v_{H}(u)
$$


are given explicitly by

$$
\begin{aligned}
& \eta_{x}=W\left(\frac{1+\nu^{2} \sin ^{2}\left(\theta_{1}-\theta_{2}\right) \eta_{z}^{2}}{\beta^{2}-W^{2}}\right)^{1 / 2}-\nu \cos \left(\theta_{1}-\theta_{2}\right) \eta_{z}, \\
& \omega_{x}=\frac{W}{(1+\eta)^{2}}\left(\frac{1+\nu^{2} \sin ^{2}\left(\theta_{1}-\theta_{2}\right) \eta_{z}^{2}}{\beta^{2}-W^{2}}\right)^{1 / 2} \int_{0}^{1} y \Phi_{y}\left(\Psi-\sin \theta_{2}\right) \mathrm{d} y \\
& +\alpha \eta-\nu^{2} \sin ^{2}\left(\theta_{1}-\theta_{2}\right)\left[\eta_{z}\left(\frac{\beta^{2}-W^{2}}{1+\nu^{2} \sin ^{2}\left(\theta_{1}-\theta_{2}\right) \eta_{z}^{2}}\right)^{1 / 2}\right]_{z} \\
& -\frac{1}{2} \sin ^{2} \theta_{2}+\left.\nu\left(\sin \theta_{2} \cos \left(\theta_{1}-\theta_{2}\right)-\sin \theta_{1}\right) \Phi_{z}\right|_{y=1}-\nu \cos \left(\theta_{1}-\theta_{2}\right) \omega_{z} \\
& +\int_{0}^{1}\left\{\frac{\left(\Psi-\sin \theta_{2}\right)^{2}}{2(1+\eta)^{2}}-\frac{\Phi_{y}^{2}}{2(1+\eta)^{2}}+\frac{1}{2} \nu^{2} \sin ^{2}\left(\theta_{1}-\theta_{2}\right)\left(\Phi_{z}^{2}-\frac{y^{2} \eta_{z}^{2} \Phi_{y}^{2}}{(1+\eta)^{2}}\right)\right. \\
& +\nu^{2} \sin ^{2}\left(\theta_{1}-\theta_{2}\right)\left(\left(\Phi_{z}-\frac{y \eta_{z} \Phi_{y}}{1+\eta)}\right) y \Phi_{y}\right)_{z} \\
& \left.+\cos \left(\theta_{1}-\theta_{2}\right) \frac{y \Phi_{y}\left(\Psi-\sin \theta_{2}\right) \nu \eta_{z}}{(1+\eta)^{2}}\right\} \mathrm{d} y, \\
& \Phi_{x}=\frac{\Psi-\sin \theta_{2}}{1+\eta}+\frac{y \Phi_{y} W}{1+\eta}\left(\frac{1+\nu^{2} \sin ^{2}\left(\theta_{1}-\theta_{2}\right) \eta_{z}^{2}}{\beta^{2}-W^{2}}\right)^{1 / 2} \\
& +\sin \theta_{2}-\nu \cos \left(\theta_{1}-\theta_{2}\right) \Phi_{z} \\
& \Psi_{x}=-\frac{\Phi_{y y}}{1+\eta}-\nu \cos \left(\theta_{1}-\theta_{2}\right) \Psi_{z} \\
& +\left((y \Psi)_{y}-\sin \theta_{2}\right) \frac{W}{1+\eta}\left(\frac{1+\nu^{2} \sin ^{2}\left(\theta_{1}-\theta_{2}\right) \eta_{z}^{2}}{\beta^{2}-W^{2}}\right)^{1 / 2} \\
& +\nu^{2} \sin ^{2}\left(\theta_{1}-\theta_{2}\right)\left(y \eta_{z}\left(\Phi_{z}-\frac{y \eta_{z} \Phi_{y}}{1+\eta}\right)\right)_{y} \\
& -\nu^{2} \sin ^{2}\left(\theta_{1}-\theta_{2}\right)\left((1+\eta)\left(\Phi_{z}-\frac{y \eta_{z} \Phi_{y}}{1+\eta}\right)\right)_{z} \cdot
\end{aligned}
$$

The results in ref. [11, Appendix A] show that the right-hand sides of equations (24)-(27) define a smooth function from $N$ into $X_{s}$ and that the boundary conditions (21), (22) are well defined. Moreover, an explicit calculation shows that a solution to (24)-(27) defines a solution of (12)(15).

The Hamiltonian system $(M, \Omega, H)$ has the conserved quantities

$$
H(\eta, \omega, \Phi, \Psi), \quad \int_{S} \omega \eta_{z} \mathrm{~d} z+\int_{\Sigma} \Psi \Phi_{z} \mathrm{~d} y \mathrm{~d} z, \quad \int_{\Sigma} \Psi \mathrm{d} y \mathrm{~d} z,
$$

which are associated with continuous symmetries, namely the invariance of equations (12)(15) under translations in $x, z$ and $\phi$ and which are inherited by Hamilton's equations (24)-(27). Hamilton's equations also inherit the discrete symmetry (16) of the hydrodynamic problem: they 
are reversible, that is the Hamiltonian vector field anticommutes with the reverser $\mathcal{R}: X_{s} \rightarrow X_{s}$ defined by

$$
\mathcal{R}(\eta(z), \omega(z), \Phi(y, z), \Psi(y, z))=(\eta(-z),-\omega(-z),-\Phi(y,-z), \Psi(y,-z)) .
$$

Hamilton's equations have additional symmetries in the special cases $\theta_{1}=0, \theta_{2}= \pm \pi / 2$ and $\theta_{1}= \pm \pi / 2, \theta_{2}=0$ identified in Section 2.1 above. In the former case Hamilton's equations are invariant under the reflection $\mathcal{S}_{1}: X_{s} \rightarrow X_{s}$ given by

$$
\mathcal{S}_{1}(\eta(z), \omega(z), \Phi(y, z), \Psi(y, z))=(\eta(-z), \omega(-z), \Phi(y,-z), \Psi(y,-z))
$$

and have the additional reversibility defined by $\mathcal{R}_{1}: X_{s} \rightarrow X_{s}$, where $\mathcal{R}_{1}(\eta, \omega, \Phi, \Psi)=$ $(\eta,-\omega,-\Phi, \Psi)$. In the latter case Hamilton's equations are invariant under the reflection $\mathcal{S}_{2}$ : $X_{s} \rightarrow X_{s}$ given by

$$
\mathcal{S}_{2}(\eta(z), \omega(z), \Phi(y, z), \Psi(y, z))=(\eta(-z), \omega(-z),-\Phi(y,-z),-\Psi(y,-z))
$$

and have the additional reversibility defined by $\mathcal{R}_{2}: X_{s} \rightarrow X_{s}$ given by $\mathcal{R}_{2}(\eta, \omega, \Phi, \Psi)=$ $(\eta,-\omega, \Phi,-\Psi)$. Notice that $\mathcal{R}=\mathcal{R}_{1} \circ \mathcal{S}_{1}$ and $\mathcal{R}=\mathcal{R}_{2} \circ \mathcal{S}_{2}$.

\subsection{Centre-manifold reduction}

Our strategy in finding solutions to the Hamiltonian system $(M, \Omega, H)$, and hence to the hydrodynamic problem (12)-(15), consists in applying a reduction principle which asserts that $(M, \Omega, H)$ is locally equivalent to a finite-dimensional Hamiltonian system. The key result is the following theorem, which is a parameterised, Hamiltonian version of a reduction principle for quasilinear evolutionary equations presented by Mielke [24, Theorem 4.1] (see Buffoni, Groves $\&$ Toland [4, Theorem 4.1]).

Theorem 1 Consider the differential equation

$$
u_{x}=\mathcal{K} u+\mathcal{N}(u ; \lambda)
$$

which represents Hamilton's equations for the Hamiltonian system $\left(M, \Omega^{\lambda}, H^{\lambda}\right)$. Here u belongs to a Hilbert space $\mathcal{X}, \lambda \in \mathbb{R}^{\ell}$ is a parameter and $\mathcal{K}: \mathcal{D}(\mathcal{K}) \subset \mathcal{X} \rightarrow \mathcal{X}$ is a densely defined, closed linear operator. Regarding $\mathcal{D}(\mathcal{K})$ as a Hilbert space equipped with the graph norm, suppose that 0 is an equilibrium point of (29) when $\lambda=0$ and that

(H1) The part of the spectrum $\sigma(\mathcal{K})$ of $\mathcal{K}$ which lies on the imaginary axis consists of a finite number of eigenvalues of finite multiplicity and that this part of $\sigma(\mathcal{K})$ is separated from the rest of $\sigma(\mathcal{K})$ in the sense of Kato, so that $X$ admits the decomposition $\mathcal{X}=\mathcal{X}_{1} \oplus \mathcal{X}_{2}$, where $\mathcal{X}_{1}=\mathcal{P}(\mathcal{X}), \mathcal{X}_{2}=(I-\mathcal{P})(\mathcal{X})$ and $\mathcal{P}$ is the spectral projection corresponding the purely imaginary part of $\sigma(\mathcal{K})$.

(H2) The operator $\mathcal{K}_{2}=\left.\mathcal{K}\right|_{\mathcal{X}_{2}}$ satisfies the estimate

$$
\left.\| \mathcal{K}_{2}-\mathrm{i} a I\right)^{-1} \|_{\mathcal{X}_{2} \rightarrow \mathcal{X}_{2}} \leq \frac{C}{1+|a|}, \quad a \in \mathbb{R}
$$

for some constant $C$ that is independent of a. 
(H3) There exists a natural number $n$ and neighbourhoods $\Lambda \subset \mathbb{R}^{\ell}$ of 0 and $U \subset \mathcal{D}(\mathcal{K})$ of 0 such that $N$ is $(n+1)$ times continuously differentiable on $U \times \Lambda$, its derivatives are bounded and uniformly continuous on $U \times \Lambda$ and $N(0,0)=0, \mathrm{~d}_{1} N[0,0]=0$.

Under these hypotheses there exist neighbourhoods $\tilde{\Lambda} \subset \Lambda$ of 0 and $\tilde{U}_{1} \subset U \cap \mathcal{X}_{1}, \tilde{U}_{2} \subset U \cap \mathcal{X}_{2}$ of 0 and a reduction function $h: \tilde{U}_{1} \times \tilde{\Lambda} \rightarrow \tilde{U}_{2}$ with the following properties. The reduction function $h$ is $k$ times continuously differentiable on $\tilde{U}_{1} \times \tilde{\Lambda}$, its derivatives are bounded and uniformly continuous on $\tilde{U}_{1} \times \tilde{\Lambda}$ and $h(0 ; 0)=0, \mathrm{~d}_{1} h[0 ; 0]=0$. The graph $M_{\mathrm{C}}^{\lambda}=\left\{u_{1}+h\left(u_{1} ; \lambda\right) \in\right.$ $\left.\tilde{U}_{1} \times \tilde{U}_{2}: u_{1} \in \tilde{U}_{1}\right\}$ is a Hamiltonian centre manifold for (29), so that

(i) $M_{\mathrm{C}}^{\lambda}$ is a locally invariant manifold of (29): through every point in $M_{\mathrm{C}}^{\lambda}$ there passes a unique solution of (29) that remains on $M_{\mathrm{C}}^{\lambda}$ as long as it remains in $\tilde{U}_{1} \times \tilde{U}_{2}$.

(ii) Every small bounded solution $u(x), x \in \mathbb{R}$ of (29) that satisfies $\left(u_{1}(x), u_{2}(x)\right) \in \tilde{U}_{1} \times$ $\tilde{U}_{2}$ lies completely in $M_{\mathrm{C}}^{\lambda}$.

(iii) Every solution $u_{1}:(a, b) \rightarrow \tilde{U}_{1}$ of the reduced equation

$$
u_{1 x}=\mathcal{K} u_{1}+\mathcal{P} \mathcal{N}\left(u_{1}+h\left(u_{1} ; \lambda\right) ; \lambda\right)
$$

generates a solution

$$
u(x)=u_{1}(x)+h\left(u_{1}(x) ; \lambda\right)
$$

of the full equation (29).

(iv) $M_{\mathrm{C}}^{\lambda}$ is a symplectic submanifold of $M$ and the flow determined by the Hamiltonian system $\left(M_{\mathrm{C}}^{\lambda}, \Omega_{\mathrm{C}}^{\lambda}, H_{\mathrm{C}}^{\lambda}\right)$, where the subscript $C$ denotes restriction to $M_{\mathrm{C}}^{\lambda}$, coincides with the flow on $M_{\mathrm{C}}^{\lambda}$ determined by $\left(M, \Omega^{\lambda}, H^{\lambda}\right)$. The reduced equation (30) represents Hamilton's equations for $\left(M_{\mathrm{C}}^{\lambda}, \Omega_{\mathrm{C}}^{\lambda}, H_{\mathrm{C}}^{\lambda}\right)$.

The next step is to write the Hamiltonian formulation of the hydrodynamic problem presented in Section 2.2 above in a form to which Theorem 1 is applicable. Write

$$
\left(\alpha, \beta, \theta_{1}, \theta_{2}, \nu\right)=\left(\alpha_{0}+\lambda_{1}, \beta_{0}+\lambda_{2}, \theta_{1}^{0}+\lambda_{3}, \theta_{2}^{0}+\lambda_{4}, \nu_{0}+\lambda_{5}\right)
$$

where $\alpha_{0}, \beta_{0}, \theta_{1}^{0}, \theta_{2}^{0}, \nu_{0}$ are fixed and $\lambda_{j}$ lies in a neighbourhood $\Lambda_{j}$ of the origin in $\mathbb{R}$, and consider solutions of Hamilton's equations for $(M, \Omega, H)$ which lie in a neighbourhood $V$ of the origin in $X_{s+1}$. Choosing $V$ and $\Lambda_{2}$ small enough so that

$$
\eta(z)>-\frac{1}{2}>-1, \quad\left|\lambda_{2}\right|<\frac{\beta_{0}}{4}, \quad|W(z)|<\frac{\beta_{0}}{2}<\beta_{0}+\lambda_{2}
$$

for all $z \in[0,2 \pi]$, where $W$ is the function defined by (19), we arrive at the equation

$$
u_{x}=f^{\lambda}(u)
$$

where $f^{\lambda}: V \rightarrow X_{s}$ is the smooth function defined by the right-hand sides of (24)-(27) with $\alpha, \beta, \theta_{1}, \theta_{2}, \nu$ replaced by the expressions in equation (32). Formula (33) represents Hamilton's equations for the Hamiltonian system $\left(M, \Omega, H^{\lambda}\right)$, where $H^{\lambda}$ is the smooth functional defined 
upon the manifold domain $V$ of $M$ by equations (18) and (32); the domain $\mathcal{D}\left(v_{H}^{\lambda}\right)$ of the Hamiltonian vector field $v_{H}^{\lambda}$ is the subset of $V$ specified by the boundary conditions (21), (22) with the parameterisation (32) and $v_{H}^{\lambda}(u)=f^{\lambda}(u)$ for each $u \in \mathcal{D}\left(v_{H}^{\lambda}\right)$.

The reduction procedure cannot be applied directly to (33) because of the nonlinear boundary condition (22). This difficulty is overcome using the following change of variable which leads to an equivalent problem in a linear space. Write $\widetilde{\lambda}=\left(\lambda_{2}, \lambda_{3}, \lambda_{4}, \lambda_{5}\right)$ and $\widetilde{\Lambda}=\Lambda_{2} \times \cdots \times \Lambda_{5}$, and define $F: V \times \widetilde{\Lambda} \subset X_{s+1} \times \mathbb{R}^{4} \rightarrow H_{\text {per }}^{s+1}(\Sigma)$ by the formula

$$
\begin{aligned}
& F(\eta, \omega, \Phi, \Psi ; \widetilde{\lambda})(y) \\
& =-\nu \sin \theta_{1} y(1+\eta) \eta_{z}+\nu \sin \theta_{2} \cos \left(\theta_{1}-\theta_{2}\right) y \eta_{z}+\nu^{2} \sin ^{2}\left(\theta_{1}-\theta_{2}\right) y \eta_{z}\left((1+\eta) \Phi_{z}-\eta_{z} \Phi_{y}\right) \\
& \quad+y W\left(\Psi-\sin \theta_{2}\right)\left(\frac{1+\nu^{2} \sin ^{2}\left(\theta_{1}-\theta_{2}\right) \eta_{z}^{2}}{\beta^{2}-W^{2}}\right)^{1 / 2},
\end{aligned}
$$

in which $\beta, \theta_{1}, \theta_{2}, \nu$ are given by (32), so that the boundary conditions (21), (22) are equivalent to

$$
\Phi_{y}=F(\eta, \omega, \Phi, \Psi ; \widetilde{\lambda}) \quad \text { on } y=0,1 .
$$

Consider the function $G^{\widetilde{\lambda}}: V \rightarrow X_{s+1}$ given by $G^{\widetilde{\lambda}}(\eta, \omega, \Phi, \Psi)=(\eta, \zeta, \Gamma, \Psi)$, where

$$
\zeta=\omega-\sin \left(\theta_{2}^{0}+\lambda_{4}\right) \int_{0}^{1} y \Phi_{y} \mathrm{~d} y, \quad \Gamma=\Phi-\chi_{y}
$$

and $\chi \in H_{\text {per }}^{s+3}(\Sigma)$ is the unique solution of the elliptic boundary-value problem

$$
\begin{aligned}
\chi_{y y}+\chi_{z z} & =F(\eta, \omega, \Phi, \Psi ; \widetilde{\lambda}) & & \text { in } \Sigma \\
\chi & =0 & & \text { on } y=0,1 .
\end{aligned}
$$

Notice that

$$
\begin{aligned}
\Gamma_{y} & =\Phi_{y}-\chi_{y y} \\
& =\Phi_{y}+\chi_{z z}-F(\eta, \omega, \Phi, \Psi ; \widetilde{\lambda})
\end{aligned}
$$

and since $\chi_{z z}=0$ on $y=0,1$, the boundary conditions (21), (22) are transformed into

$$
\Gamma_{y}=0 \quad \text { on } y=0,1 \text {. }
$$

The following lemma shows that $G^{\tilde{\lambda}}$ defines a valid change of variable (cf. Lemma 4 in ref. [11]).

\section{Lemma 2}

(i) For each $\tilde{\lambda} \in \widetilde{\Lambda}$ the mapping $G^{\tilde{\lambda}}$ is a smooth diffeomorphism from a neighbourhood $V$ of 0 in $X_{s+1}$ onto a neighbourhood $\widetilde{V}$ of 0 in $X_{s+1}$. The mappings $G^{\widetilde{\lambda}}$ and $\left(G^{\tilde{\lambda}}\right)^{-1}$ and their derivatives depend smoothly upon $\widetilde{\lambda} \in \widetilde{\Lambda}$.

(ii) For each $(u, \widetilde{\lambda}) \in V \times \widetilde{\Lambda}$ the operator $\mathrm{d} G^{\widetilde{\lambda}}[u]: X_{s+1} \rightarrow X_{s+1}$ extends to an isomorphism $\widetilde{\mathrm{d} G} \widetilde{\lambda}[u]: X_{s} \rightarrow X_{s}$. The operators $\widetilde{\mathrm{d} G} \widetilde{\lambda}[u],\left(\widetilde{\mathrm{d} G}^{\widetilde{\lambda}}[u]\right)^{-1} \in \mathcal{L}\left(X_{s}, X_{s}\right)$ depend smoothly upon $(u, \widetilde{\lambda}) \in V \times \widetilde{\Lambda}$. 
A simple calculation shows that the diffeomorphism $G^{\widetilde{\lambda}}$ transforms (33) into

$$
v_{x}=g^{\lambda}(v),
$$

where $g^{\lambda}: \widetilde{V} \rightarrow X_{s}$ is the smooth vector field defined by

$$
g^{\lambda}(v)=\widetilde{\mathrm{d}}^{\widetilde{\lambda}}\left[\left(G^{\widetilde{\lambda}}\right)^{-1}(v)\right]\left(f^{\lambda}\left(\left(G^{\tilde{\lambda}}\right)^{-1}(v)\right)\right)
$$

Formula (36) represents Hamilton's equations for the Hamiltonian system $\left(M, \widetilde{\Omega}^{\widetilde{\lambda}}, \widetilde{H}^{\lambda}\right)$, where $\widetilde{\Omega}^{\widetilde{\lambda}}$ and $\widetilde{H}^{\lambda}$ are defined on the manifold domain $\widetilde{V}$ of $M$ by

$$
\left.\widetilde{\Omega}^{\widetilde{\lambda}}\right|_{m}\left(v_{1}, v_{2}\right)=\Omega\left(\widetilde{\mathrm{d} G} \widetilde{\lambda}^{\widetilde{\lambda}}\left[\left(G^{\tilde{\lambda}}\right)^{-1}(m)\right]^{-1}\left(v_{1}\right), \widetilde{\mathrm{d} G} \widetilde{\lambda}^{\widetilde{\lambda}}\left[\left(G^{\tilde{\lambda}}\right)^{-1}(m)\right]^{-1}\left(v_{2}\right)\right)
$$

for $\widetilde{\lambda} \in \widetilde{\Lambda}, v_{1},\left.v_{2} \in T M\right|_{m}$ and

$$
\widetilde{H}^{\lambda}(m)=H^{\lambda}\left(\left(G^{\widetilde{\lambda}}\right)^{-1}(m)\right)
$$

The domain $\mathcal{D}\left(v_{\widetilde{H}}^{\lambda}\right)$ of the Hamiltonian vector field is the subset of $\widetilde{V}$ given by the linear boundary conditions (35) and $v_{\widetilde{H}}^{\lambda}(v)=g^{\lambda}(v)$ for any $v \in \mathcal{D}\left(v_{\widetilde{H}}^{\lambda}\right)$.

We consider (36) as a quasilinear evolutionary equation

$$
v_{x}=\mathcal{K} v+\mathcal{N}(v ; \lambda)
$$

in the Hilbert space $\mathcal{X}=X_{s}$ to which we apply Theorem 1 ; here $\mathcal{K}=\mathrm{d} g^{0}[0], \mathcal{D}(\mathcal{K})=$ $\left\{(\eta, \zeta, \Gamma, \Psi) \in X_{s+1}:\left.\Gamma_{y}\right|_{y=0}=\left.\Gamma_{y}\right|_{y=1}=0\right\}$ and $\mathcal{N}(u ; \lambda)=g^{\lambda}(v)-\mathcal{K} v$, so that $\mathcal{K}$ is densely defined and $\mathcal{N}: \tilde{V} \rightarrow \mathcal{X}$ is smooth. The remaining spectral hypotheses on $\mathcal{K}$ are verified by studying the formal linearisation of $f^{0}$ at 0 , namely the operator $\mathcal{L}: \mathcal{D}(\mathcal{L}) \subset \mathcal{X} \rightarrow \mathcal{X}$ defined by

$$
\mathcal{L}(\eta, \omega, \Phi, \Psi)=\left(\eta_{1}, \omega_{1}, \Phi_{1}, \Psi_{1}\right)
$$

in which

$$
\begin{aligned}
\eta_{1}= & \frac{1}{\beta_{0}}\left(\omega-\sin \theta_{2}^{0} \int_{0}^{1} y \Phi_{y} \mathrm{~d} y\right)-\nu_{0} \cos \left(\theta_{1}^{0}-\theta_{2}^{0}\right) \eta_{z}, \\
\omega_{1}= & \left(\alpha_{0}-\sin ^{2} \theta_{2}^{0}\right) \eta-\nu_{0}^{2} \beta_{0} \sin ^{2}\left(\theta_{1}^{0}-\theta_{2}^{0}\right) \eta_{z z}-\nu_{0} \cos \left(\theta_{1}-\theta_{2}\right) \omega_{z}-\sin \theta_{2} \int_{0}^{1} \Psi \mathrm{d} y \\
& +\left.\nu_{0}\left(\sin \theta_{2} \cos \left(\theta_{1}-\theta_{2}\right)-\sin \theta_{1}\right) \Phi_{z}\right|_{y=1}, \\
\Phi_{1}= & \Psi+\sin \theta_{2}^{0} \eta-\nu_{0} \cos \left(\theta_{1}^{0}-\theta_{2}^{0}\right) \Phi_{z}, \\
\Psi_{1}= & -\Phi_{y y}-\nu_{0}^{2} \sin ^{2}\left(\theta_{1}^{0}-\theta_{2}^{0}\right) \Phi_{z z}-\nu_{0} \cos \left(\theta_{1}^{0}-\theta_{2}^{0}\right) \Psi_{z}-\frac{\sin \theta_{2}^{0}}{\beta_{0}}\left(\omega-\sin \theta_{2}^{0} \int_{0}^{1} y \Phi_{y} \mathrm{~d} y\right) ;
\end{aligned}
$$


the domain $\mathcal{D}(L)$ of $\mathcal{L}$ is the subspace of elements of $X_{s+1}$ that satisfy the linear boundary conditions

$$
\begin{array}{ll}
\Phi_{y}=0 & \text { on } y=0 \\
\Phi_{y}=\nu_{0}\left(\sin \theta_{2}^{0} \cos \left(\theta_{1}^{0}-\theta_{2}^{0}\right)-\sin \theta_{1}\right) \eta_{z}-\frac{\sin \theta_{2}^{0}}{\beta_{0}}\left(\omega-\sin \theta_{2}^{0} \int_{0}^{1} y \Phi_{y} \mathrm{~d} y\right) & \text { on } y=1 .
\end{array}
$$

The arguments given by Groves $\&$ Mielke $[11, \S 3.3]$ show that the spectral properties of $\mathcal{K}$ and $\mathcal{L}$ are identical.

\section{Lemma 3}

(i) The spectrum $\sigma(\mathcal{K})$ of $\mathcal{K}$ consists entirely of isolated eigenvalues of finite algebraic multiplicity and $\sigma(\mathcal{K}) \cap i \mathbb{R}$ is a finite set. A complex number $\sigma$ is an eigenvalue of $\mathcal{K}$ with corresponding eigenvectors in the nth Fourier mode if and only if

$$
\left(\sigma \sin \theta_{2}^{0}+\mathrm{i} n \nu_{0} \sin \theta_{1}^{0}\right)^{2} \cos \gamma=\left(\alpha_{0}-\beta_{0} \gamma^{2}\right) \gamma \sin \gamma
$$

where $\gamma^{2}=\sigma^{2}-n^{2} \nu_{0}^{2}+2 \cos \left(\theta_{1}^{0}-\theta_{2}^{0}\right)$ in $\nu_{0} \sigma$. Moreover, the set $\sigma(\mathcal{K})$ is symmetric with respect to the real and imaginary axis, that is $\sigma(\mathcal{K})=\overline{\sigma(\mathcal{K})}=-\sigma(\mathcal{K})$.

(ii) There exist real constants $C, q_{0}>0$ such that

$$
\left\|(\mathcal{K}-\mathrm{i} q I)^{-1}\right\|_{\mathcal{X} \rightarrow \mathcal{X}} \leq \frac{C}{|q|}
$$

for each real number $q$ with $|q|>q_{0}$.

The above discussion shows that the reduction result in Theorem 1 may be applied at any parameter values for which $\mathcal{K}$ has at least one purely imaginary eigenvalue. The Hamiltonian centre manifold $M_{\mathrm{C}}^{\lambda}$, which is of class $k$ for any fixed $k \in \mathbb{N}$, is equipped with the single coordinate chart $\tilde{U}_{1} \subset X_{1}$ and coordinate map $\phi: M_{\mathrm{C}}^{\lambda} \rightarrow \tilde{U}_{1}$ defined by

$$
\phi^{-1}\left(u_{1}\right)=u_{1}+h\left(u_{1} ; \lambda\right) \text {. }
$$

In the following calculations it is more convenient to change to the coordinate chart $\widetilde{W}_{1}=$ $\left(\mathrm{d} G^{0}[0]\right)^{-1}\left(\widetilde{U}_{1}\right)$, so that $\widetilde{W}_{1}$ is a neighbourhood of the origin in the centre subspace of the linear operator $L$ and to redefine the coordinate map by

$$
\psi^{-1}\left(w_{1}\right)=w_{1}+\check{h}\left(w_{1} ; \lambda\right)
$$

where $\check{h}: W_{1} \times \widetilde{\Lambda} \rightarrow V$ is given by

$$
\check{h}\left(w_{1} ; \lambda\right)=\left(G^{\widetilde{\lambda}}\right)^{-1}\left(\mathrm{~d} G^{0}[0] w_{1}+h\left(\mathrm{~d} G^{0}[0] w_{1} ; \lambda\right)\right)-w_{1} .
$$


In this coordinate system $M_{\mathrm{C}}^{\lambda}$ is $\left\{w_{1}+\check{h}\left(w_{1} ; \lambda\right) \in V: w_{1} \in W_{1}\right\}$; it lies in $\mathcal{D}\left(v_{H}^{\lambda}\right)$ and defines a centre manifold for the Hamiltonian system $\left(M, \Omega, H^{\lambda}\right)$. The reduced Hamiltonian $H_{\mathrm{C}}^{\lambda}$ and the reduced 2 -form $\Omega_{\mathrm{C}}^{\lambda}$ are given by

$$
\begin{aligned}
H_{\mathrm{C}}^{\lambda}\left(w_{1}\right) & =H^{\lambda}\left(w_{1}+\check{h}\left(w_{1} ; \lambda\right)\right), \\
\left.\Omega_{\mathrm{C}}^{\lambda}\right|_{w_{1}}\left(v^{1}, v^{2}\right) & =\left.\Omega\right|_{w_{1}+\check{h}\left(w_{1} ; \lambda\right)}\left(v^{1}+\mathrm{d}_{1} \check{h}\left[w_{1} ; \lambda\right]\left(v^{1}\right), v^{2}+\mathrm{d}_{1} \check{h}\left[w_{1} ; \lambda\right]\left(v^{2}\right)\right) \\
& =\Omega\left(v_{1}, v_{2}\right)+\mathcal{O}\left(\left|\left(w_{1}, \lambda\right) \| v^{1}\right|\left|v^{2}\right|\right),
\end{aligned}
$$

and $\Omega_{\mathrm{C}}^{\lambda}$ can be transformed into $\Omega$ by a near-identity Darboux change of coordinates (cf. Groves $\&$ Mielke [11, Theorem 4]). One can always choose a basis for $W_{1}$ so that $\Omega$ is the canonical symplectic 2 -form $\Upsilon$ in this coordinate system (a 'symplectic basis'); the reduction procedure therefore delivers a finite-dimensional canonical Hamiltonian system.

\section{A catalogue of bifurcation scenarios}

\subsection{Purely imaginary eigenvalues}

According to Lemma 3(i) the purely imaginary number i $\kappa$ is an eigenvalue of $\mathcal{K}$ with corresponding eigenvectors in the $n$th Fourier mode (a 'mode $n$ eigenvalue') if and only if

$$
\left(\sin \theta_{2} \kappa+\sin \theta_{1} n \nu\right)^{2}=\left(\alpha+\beta \widetilde{\gamma}^{2}\right) \widetilde{\gamma} \tanh \widetilde{\gamma}
$$

where $\widetilde{\gamma}^{2}=\kappa^{2}+n^{2} \nu^{2}+2 \cos \left(\theta_{1}-\theta_{2}\right) n \nu \kappa$ (for convenience we drop the sub- and superscripts 0 in this section). There is a simple connection between this equation and the classical dispersion relation for the Euler equations (5)-(8). Clearly $\sigma=\mathrm{i} \kappa$ is a mode $n$ eigenvalue of $\mathcal{K}$ if and only if the equation

$$
\mathcal{K} u=\mathrm{i} \kappa u
$$

has a nontrivial solution $u$ of the form $u=u_{\kappa, n} \mathrm{e}^{\mathrm{i} n z}$ or equivalently if and only if the linear equation

$$
u_{x}=\mathcal{K} u
$$

has a nontrivial solution of the form $u=u_{\kappa, n} e^{\mathrm{i} \kappa x+\mathrm{i} n z}$. Such a solution corresponds to a solution of the linearised Euler equations (5)-(8) of the special form

$$
\Phi(X, Y, Z)=\Phi_{\kappa, n}(Y) e^{\mathrm{i} k X+\mathrm{i} \ell Z}, \quad \eta(X, Z)=\eta_{\kappa, n} e^{\mathrm{i} k X+\mathrm{i} \ell Z}
$$

with

$$
k=\sin \theta_{2} \kappa+\sin \theta_{1} n \nu, \quad \ell=-\cos \theta_{2} \kappa-\cos \theta_{1} n \nu,
$$

and it is well known that a solution of this kind exists if and only if $k$ and $\ell$ satisfy the classical dispersion relation

$$
\mathcal{D}(k, \ell)=-k^{2}+\left(\alpha+\beta\left(k^{2}+\ell^{2}\right)\right) \sqrt{k^{2}+\ell^{2}} \tanh \sqrt{k^{2}+\ell^{2}}=0 .
$$

We conclude that $\sigma=\mathrm{i} \kappa$ is a mode $n$ eigenvalue of $\mathcal{K}$ if and only if the numbers $k$ and $\ell$ given by equation (40) satisfy $\mathcal{D}(k, \ell)=0$. 
The above observation has an elegant geometric interpretation: purely imaginary eigenvalues i $\kappa$ of $\mathcal{K}$ correspond to intersections in the $(k, \ell)$-plane of the real branches $C_{\mathrm{dr}}$ of the dispersion relation (41) with the lines $K_{n}, n=0, \pm 1, \pm 2, \ldots$ given in parametric form by

$$
K_{n}=\left\{(k, \ell) \in \mathbb{R}^{2}: k=\sin \theta_{2} \kappa+\sin \theta_{1} n \nu, \ell=-\cos \theta_{2} \kappa-\cos \theta_{1} n \nu, \kappa \in \mathbb{R}\right\} .
$$

A point of intersection of $K_{n}$ and $\mathcal{D}(k, \ell)$ corresponds to a purely imaginary mode $n$ eigenvalue $\mathrm{i} \kappa$ of $\mathcal{K}$; its imaginary part is the value of the parameter $\kappa$ in (42) at the point of intersection. The geometric multiplicity of the eigenvalue $i \kappa$ is given by the number of distinct lines in the family $\left\{K_{n}\right\}$ which intersect $C_{\mathrm{dr}}$ at this parameter value, and a tangent intersection between $K_{n}$ and $C_{\mathrm{dr}}$ indicates that each eigenvector in mode $n$ has an associated Jordan chain of length 2.

The real branches of the dispersion relation (41) have been studied by Haragus \& Pego [14]. They are curves in the $(k, \ell)$-plane given by

$$
C_{\mathrm{dr}}=\left\{(k, \ell) \in \mathbb{R}^{2}: k= \pm \sqrt{\left(\alpha+\beta a^{2}\right) a \tanh a}, l= \pm \sqrt{a^{2}-\left(\alpha+\beta a^{2}\right) a \tanh a}, a \in \mathbb{R}\right\}
$$

whose shape is shown in Figure 2(a) (insets) in the indicated regions of the $(\beta, \alpha)$-parameter plane. Notice that $C_{\mathrm{dr}}$ is symmetric with respect to the $k$-and $\ell$-axes (a consequence of the discrete symmetries (9), (10) of the Euler equations) and always contains the point $(k, \ell)=(0,0)$ (a consequence of the invariance $\phi \rightarrow \phi+c$ in the Euler equations); the eigenvalue corresponding to this intersection between $C_{\mathrm{dr}}$ and $K_{0}$ is later eliminated by a symmetry reduction and is therefore ignored in the discussion in the remainder of this section. We find that $C_{\mathrm{dr}}=\{0\}$ in region I. In region II the equation $\mathcal{D}(k, 0)=0$ has one pair of simple nonzero roots $\pm k_{\mathrm{c}}$. The real branches of the dispersion relation have the limiting behaviour

$$
\ell^{2} \sim \alpha_{1}\left(k-k_{\mathrm{c}}\right), \quad \alpha_{1}=\frac{2 k_{\mathrm{c}}\left(\sinh \left(2 k_{\mathrm{c}}\right)-2 k_{\mathrm{c}}-2 \beta k_{\mathrm{c}} \tanh k_{\mathrm{c}} \sinh \left(2 k_{\mathrm{c}}\right)\right)}{\sinh \left(2 k_{\mathrm{c}}\right)+2 k_{\mathrm{c}}+2 \beta k_{\mathrm{c}} \tanh k_{\mathrm{c}} \sinh \left(2 k_{\mathrm{c}}\right)}
$$

as $k \rightarrow k_{\mathrm{c}}$; in a neighbourhood of $(0,0)$ they have the limiting behaviour

$$
\ell^{2} \sim\left(\alpha^{-1}-1\right) k^{2}+\left(\frac{1}{3}-\frac{\beta}{\alpha}\right) \alpha^{-2} k^{4}
$$

as $k \rightarrow 0$ and therefore make angles $\pm \phi_{\alpha}=\arctan ^{-1}\left(\alpha^{-1}-1\right)^{1 / 2}$ with the $k$-axis at the origin. Notice the qualitative difference in the shape of $C_{\mathrm{dr}}$ according to the size of $\beta / \alpha$ : for $\beta / \alpha>1 / 3$ the portion $C_{\mathrm{dr}}^{+}$of $C_{\mathrm{dr}}$ in the positive quadrant is concave, but a point of inflection emerges from the origin as $\beta / \alpha$ is decreased through this critical value, and $C_{\mathrm{dr}}^{+}$is convex between the origin and this point of inflection.

In region III the equation $\mathcal{D}(k, 0)=0$ has two pairs of simple roots $\pm k_{\mathrm{c}}^{1}, \pm k_{\mathrm{c}}^{2}$, and as $k \rightarrow k_{\mathrm{c}}^{j}$ the real branches of the dispersion relation have the limiting behavior in (43). Passing from region II into region III through the line $\{\alpha=1\}$, one finds that a second point of inflection emerges from the origin, and $C_{\mathrm{dr}}^{+}$is concave to the left of the new point of inflection, convex between the two points of inflection and concave to the right of the old point of inflection. For each fixed $\beta$ the two points of inflection merge and disappear as $\alpha$ is increased, so that $C_{\mathrm{dr}}^{+}$is again concave. The curves $\Gamma$ and $\{\alpha=1\}$ separating the three regions I, II and III consist of those values of $(\beta, \alpha)$ for which the equation $\mathcal{D}(k, 0)=0$ has double roots, namely $k= \pm k^{*} \neq 0$ for $(\beta, \alpha) \in \Gamma$ and $k=0$ for $\alpha=1$. Solutions $k$ of $\mathcal{D}(k, 0)=0$ correspond to purely imaginary 
eigenvalues $i k$ of the linear operator in the spatial dynamics formulation of the two-dimensional steady water-wave problem, so that $\Gamma \cup\{\alpha=1\}$ is the set of parameter values associated with changes in the number of such eigenvalues (see Kirchgässner [20]); these parameter values are associated with bifurcation phenomena in the two-dimensional steady water-wave problem.

For given $\nu, \theta_{1}$ and $\theta_{2}$ the lines $K_{n}$ in the $(k, \ell)$-plane are parallel, equidistant and form an angle $\theta_{2}$ with the positive $\ell$-axis. They pass through the points $P_{n}=\left(\sin \theta_{1} n \nu,-\cos \theta_{1} n \nu\right)$, $n \in \mathbb{Z}$ on the line

$$
L=\left\{(k, \ell) \in \mathbb{R}^{2}: k=\sin \theta_{1} \mu, \ell=-\cos \theta_{1} \mu, \mu \in \mathbb{R}\right\}
$$

which passes through the origin and makes an angle $\theta_{1}$ with the positive $\ell$-axis (see Figure 2(b)). Observe that $C_{\mathrm{dr}}$ depends only upon $\alpha$ and $\beta$ and the number of points in the set $K_{0} \cap C_{\mathrm{dr}}$ depends only upon $\alpha, \beta$ and $\theta_{2}$, which determines the slope of each line $K_{n}$. Furthermore, for fixed $\alpha, \beta$ and $\theta_{2}$ the number of points in the sets $K_{n} \cap C_{\mathrm{dr}}, n= \pm 1, \pm 2, \ldots$ depends only upon $\nu$, which determines the distance between the lines $K_{n}$. At each fixed point of $(\beta, \alpha)$ parameter space the number of purely imaginary eigenvalues of the linear operator $\mathcal{K}$ therefore depends upon the two parameters $\theta_{2}$ and $\nu$; the third parameter $\theta_{1}$, which specifies the slope of the line $L$, influences only the values of these eigenvalues and their relative positions on the imaginary axis: the imaginary part of a purely imaginary eigenvalue corresponding to an intersection of $K_{n}$ and $C_{\mathrm{dr}}$ is the value of $K_{0}$ in the $\left(K_{0}, L\right)$-coordinate system at the intersection (the signed distance between the intersection and the point $P_{n}$ ). Finally, notice that the sets $K_{n} \cap C_{\mathrm{dr}}$ and $\mathcal{K}_{-n} \cap C_{\mathrm{dr}}$ have the same cardinality: the purely imaginary number i $\kappa$ is a mode $n$ eigenvalue if and only if the purely imaginary number $-\mathrm{i} \kappa$ is a mode $-n$ eigenvalue.

\subsection{Bifurcation scenarios involving mode 0 eigenvalues}

According to Figure 2 it is, at each point in regions II and III of $(\beta, \alpha)$ parameter space, possible to choose $\theta_{2}$ so that the line $K_{0}$ intersects $C_{\mathrm{dr}}$ in at least two nonzero points in the $(k, \ell)$-plane; these intersections imply that the linear operator $\mathcal{K}$ has a plus-minus pair of purely imaginary eigenvalues. Moreover, for each fixed value of $(\beta, \alpha)$ it is possible to choose $\nu$ large enough so that no other line $K_{n}, n \neq 0$ intersects $C_{\mathrm{dr}}$, so that the only purely imaginary eigenvalues are mode 0 eigenvalues. We are particularly interested in parameter values at which the number of purely imaginary eigenvalues changes, since these points are associated with nonlinear bifurcation phenomena. In this section we catalogue possible bifurcation scenarios of this kind which involve only mode 0 eigenvalues by fixing the values of the physical parameters $(\beta, \alpha)$ (in region II or III), choosing a value of $\nu$ which ensures that $K_{n}, n \neq 0$ does not intersect $C_{\mathrm{dr}}$ and varying the angle $\theta_{2}$. The result is shown in Figure 4 .

The basic bifurcation scenario in region III is a Hamiltonian-Hopf bifurcation (Figure 4(a)), in which a plus-minus pair of geometrically simple purely imaginary eigenvalues, each with a Jordan chain of length 2 , are created when $K_{0}$ is tangent to $C_{\mathrm{dr}}$; their magnitude is given by the distance along the lines $K_{0}$ between the tangency and the origin $L$. Figure 4(a) shows that a Hamiltonian-Hopf bifurcation can also occur at certain points in region II for $\beta / \alpha<1 / 3$. (Notice that, although $C_{\mathrm{dr}}^{+}$is not always concave, for fixed $(\beta, \alpha)$ the line $K_{0}$ is tangent to $C_{\mathrm{dr}}$ at a pair of nonzero points for at most one value of $\theta_{2}$.) In region II an additional bifurcation scenario occurs when the line $K_{0}$ is tangent to $C_{\mathrm{dr}}$ at the origin, that is when $\theta_{2}= \pm\left(\pi / 2 \pm \phi_{\alpha}\right)$. Here a 

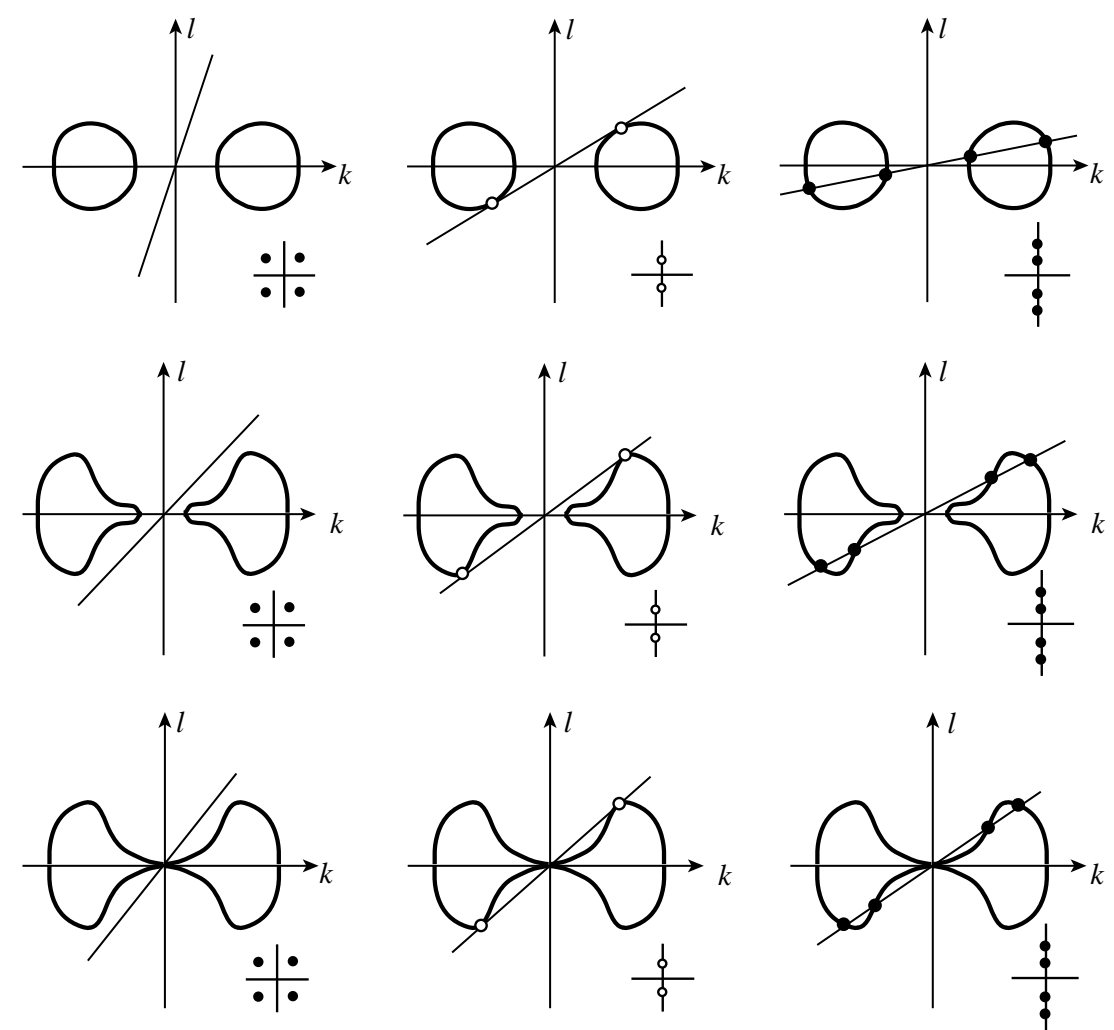

(a) The number of purely imaginary mode 0 eigenvalues increases from zero to four in a Hamiltonian-Hopf bifurcation as $K_{0}$ becomes tangent to $C_{\mathrm{dr}}$ at two nonzero points.
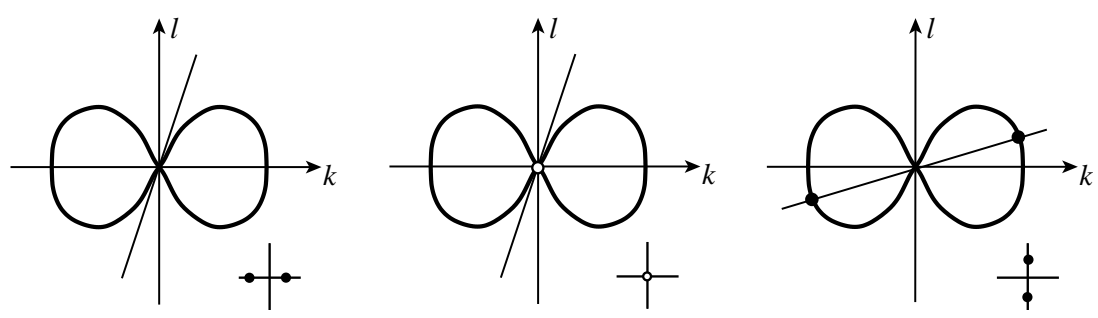

(b) The number of purely imaginary mode 0 eigenvalues increases from zero to two in a Hamiltonian $0^{2}$-resonance as $K_{0}$ becomes tangent to $C_{\mathrm{dr}}$ at the origin.
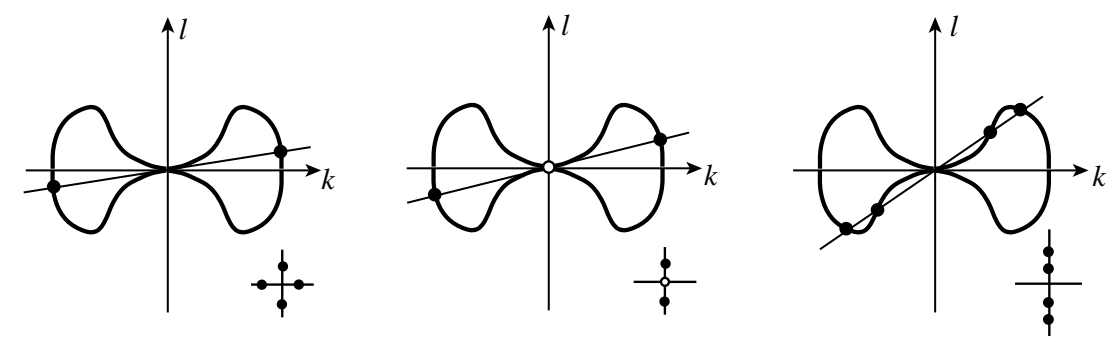

(c) The number of purely imaginary mode 0 eigenvalues increases from two to four in a Hamiltonian $0^{2} \mathrm{i} \omega$-resonance as $K_{0}$ becomes tangent to $C_{\mathrm{dr}}$ at the origin.

Figure 4: Bifurcation scenarios involving only mode 0 eigenvalues; the value of $(\beta, \alpha)$ is fixed and $\theta_{2}$ is varied. The solid dots represent simple eigenvalues while the hollow dots represent geometrically simple eigenvalues whose eigenvectors have associated Jordan chains of length 2. 


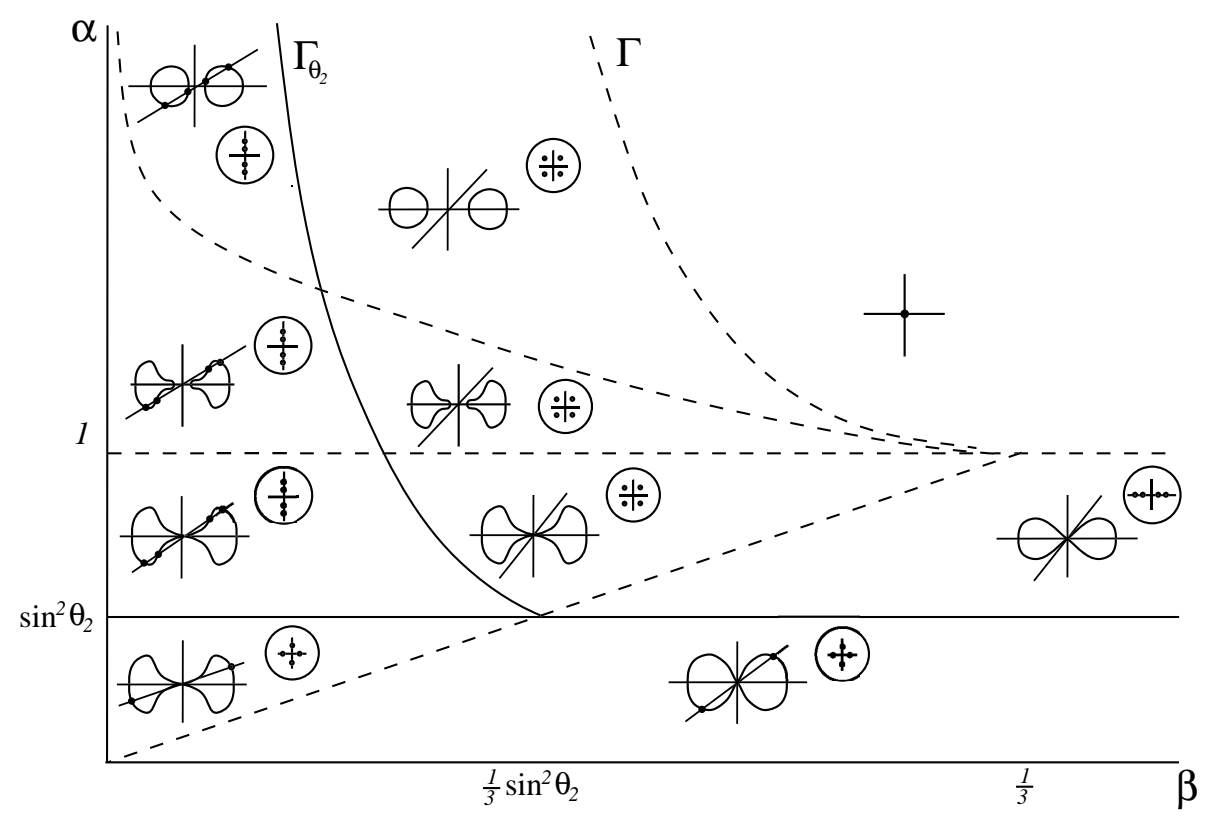

Figure 5: Behaviour of mode 0 eigenvalues in the $(\beta, \alpha)$-plane for a fixed value of $\theta_{2}$. Solid and dashed curves denote respectively bifurcation curves and curves where the qualitative properties of $C_{\mathrm{dr}}$ changes.

geometrically simple zero eigenvalue with a Jordan chain of length 2 is created in a Hamiltonian $0^{2}$ resonance $(\beta / \alpha>1 / 3$, Figure $4(\mathrm{~b}))$ or in a Hamiltonian $0^{2} \mathrm{i} \omega$ resonance $(\beta / \alpha<1 / 3$, Figure $4(\mathrm{c}))$.

It is also instructive to examine the complementary point of view in which one fixes $\theta_{2}$ and looks for values of $(\beta, \alpha)$ at which bifurcations occur. Figure 5 shows the behaviour of mode 0 eigenvalues at points in $(\beta, \alpha)$ parameter space for fixed $\theta_{2}$. In particular, the number of purely imaginary mode 0 eigenvalues changes at points on the line $\left\{\alpha=\sin ^{2} \theta_{2}\right\}$, where two real eigenvalues become purely imaginary by passing through the origin, and on the curve $\Gamma_{\theta_{2}}$ given in parametric form by

$$
\Gamma_{\theta_{2}}=\left\{(\beta, \alpha)=\left(\frac{-\sin ^{2} \theta_{2}}{2 \sinh ^{2} \kappa}+\frac{\sin ^{2} \theta_{2}}{2 \kappa \tanh \kappa}, \frac{\kappa^{2} \sin ^{2} \theta_{2}}{2 \sinh ^{2} \kappa}+\frac{\kappa \sin ^{2} \theta_{2}}{2 \tanh \kappa}\right): \kappa \in[0, \infty)\right\},
$$

where four complex eigenvalues become purely imaginary without passing through the origin. Choosing $(\beta, \alpha)$ on one of the curves $\left\{\alpha=\sin ^{2} \theta_{2}, \beta>1 / 3\right\},\left\{\alpha=\sin ^{2} \theta_{2}, \beta>1 / 3\right\}$ or $\Gamma_{\theta_{2}}$ and choosing $\nu$ sufficiently large (depending upon $(\beta, \alpha)$ ), one obtains respectively a $0^{2}$ resonance, a $0^{2} \mathrm{i} \omega$ resonance and a Hamiltonian-Hopf bifurcation point. In the special case $\theta_{1}=0$, $\theta_{2}= \pm \pi / 2$ ( $Z$-independent waves), we recover the bifurcation diagram for the two-dimensional steady water-wave problem presented by Kirchgässner [20]. 

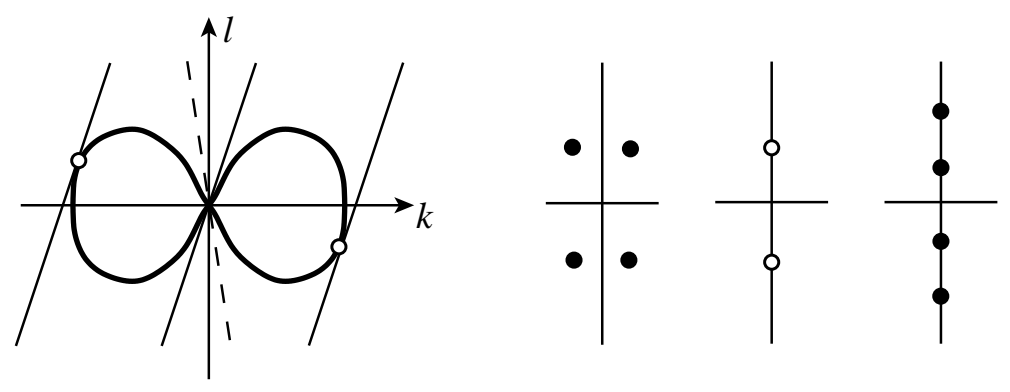

$\mathrm{v}=\mathrm{v}_{\mathrm{c}}$

$\mathrm{v}>\mathrm{v}_{\mathrm{c}} \quad \mathrm{v}=\mathrm{v}_{\mathrm{c}} \quad \mathrm{v}<\mathrm{v}_{\mathrm{c}}$

Case $I I_{\mathrm{a}}$ : the number of purely imaginary eigenvalues increases from zero to four in a Hamiltonian-Hopf bifurcation.

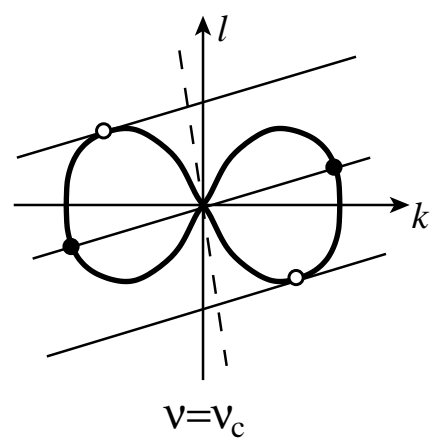

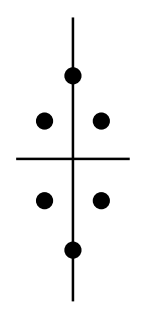

$v>v_{c}$

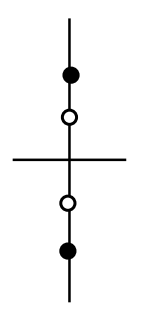

$\mathrm{v}=\mathrm{v}_{\mathrm{c}}$

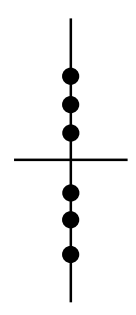

$\mathrm{v}<\mathrm{v}_{\mathrm{c}}$

Case $I_{\mathrm{b}}$ : the number of purely imaginary eigenvalues increases from two to six in a Hamiltonian $\left(\mathrm{i} \omega_{0}\right)^{2} \mathrm{i} \omega_{1}$ resonance.

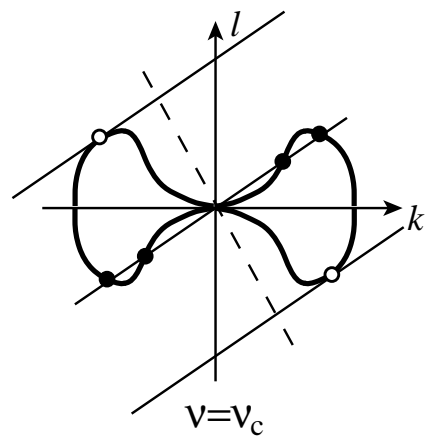

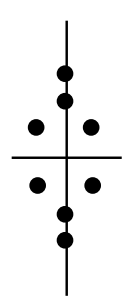

$v>v_{c}$
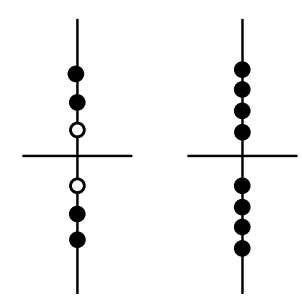

$\mathrm{v}=\mathrm{v}_{\mathrm{c}} \quad \mathrm{v}<\mathrm{v}_{\mathrm{c}}$

Case $I I_{\mathrm{c}}$ : the number of purely imaginary eigenvalues increases from four to eight in a Hamiltonian $\left(\mathrm{i} \omega_{0}\right)^{2} \mathrm{i} \omega_{1} \mathrm{i} \omega_{2}$ resonance.

Figure 6: Basic bifurcation scenarios in region II. The diagrams on the left show the lines $K_{0}$, $K_{1}, K_{-1}$ (solid) and $L$ (dashed) and the curve $C_{\mathrm{dr}}$ in the $(k, \ell)$-plane. The diagrams on the right show the eigenvalues of the linear operator $\mathcal{K}$; the solid dots represent simple eigenvalues while the hollow dots represent geometrically simple eigenvalues whose eigenvectors have associated Jordan chains of length 2. 


\subsection{Further bifurcation scenarios}

In this section we identify bifurcation scenarios by fixing the values of the physical parameters $(\beta, \alpha)$ (in region II or III) and the angles $\theta_{1}, \theta_{2}$ and varying the frequency $\nu$ : by decreasing a large initial value of $\nu$ one finds a sequence of critical frequencies

$$
\nu=\nu_{\mathrm{c}}, \frac{\nu_{\mathrm{c}}}{2}, \frac{\nu_{\mathrm{c}}}{3}, \ldots
$$

at which the pairs of lines $\left(K_{1}, K_{-1}\right),\left(K_{2}, K_{-2}\right),\left(K_{3}, K_{-3}\right), \ldots$ touch the dispersion curve $C_{\mathrm{dr}}$ in the $(k, \ell)$-plane for the first time. In particular, we catalogue the bifurcation scenarios in which only the first pair $\left(K_{1}, K_{-1}\right)$ of lines is involved.

There are three basic bifurcation scenarios in region II, both of which occur at the critical value $\nu=\nu_{\mathrm{c}}$, when the lines $K_{1}$ and $K_{-1}$ are tangent to $C_{\mathrm{dr}}$ in the $(k, \ell)$-plane (Figure 6). A plus-minus pair of geometrically simple purely imaginary eigenvalues, each with a Jordan chain of length 2 , are created at $\nu=\nu_{\mathrm{c}}$; their magnitude is given by the distance along the lines $K_{1}$ and $K_{-1}$ between the tangency and the line $L$. The cases are distinguished according to whether the line $K_{0}$ intersects $C_{\mathrm{dr}}$ in zero, two or four points. In case $\mathrm{II}_{\mathrm{a}}$ the line $K_{0}$ does not intersect $C_{\mathrm{dr}}$, and hence there are no further purely imaginary eigenvalues (a Hamiltonian-Hopf bifurcation); in case $\mathrm{II}_{\mathrm{b}}$ the line $K_{0}$ intersects $C_{\mathrm{dr}}$ in two nonzero points, so that there is an additional plus-minus pair of simple purely imaginary eigenvalues (a Hamiltonian $\left(\mathrm{i} \omega_{0}\right)^{2} \mathrm{i} \omega_{1}$ resonance); and in case $\mathrm{II}_{\mathrm{c}}$ the line $K_{0}$ intersects $C_{\mathrm{dr}}$ in four nonzero points, so that there are two additional plus-minus pairs of simple purely imaginary eigenvalues (a Hamiltonian $\left(\mathrm{i} \omega_{0}\right)^{2} \mathrm{i} \omega_{1} \mathrm{i} \omega_{2}$ resonance). The transitions between the above cases occur when the mode 0 eigenvalues undergo one of the bifurcations described in Section 3.2 above. Turning to region III, notice that there are two basic bifurcation scenarios, namely those described above for cases $\mathrm{II}_{\mathrm{a}}$ and $\mathrm{II}_{\mathrm{c}}$, which will henceforth be termed cases $\mathrm{III}_{\mathrm{a}}$ and $\mathrm{III}_{\mathrm{c}}$.

The relative position of the eigenvalues on the imaginary axis at bifurcation points is determined by the value of $\theta_{1}$. In particular, there are special values of $\theta_{1}$ for which purely imaginary eigenvalues with eigenvectors in different Fourier modes have the same value. Although the geometric multiplicity of the new eigenvalue is increased in this situation, the overall structure of the central subspace remains the same: eigenvectors in different Fourier modes remain linearly independent and the existence and length of Jordan chains is unaffected. Figure 7(a) shows an example of case $\mathrm{II}_{\mathrm{a}}$ which is degenerate in the above sense. Whenever the line $L$ passes through the points of intersection of $K_{1}$ with $C_{\mathrm{dr}}$ and $K_{-1}$ with $C_{\mathrm{dr}}$ the values of the purely eigenvalues with eigenvectors in modes 1 and -1 are both zero, so that zero is now a geometrically double eigenvalue; each eigenvector has a Jordan chain of length 2. A further degeneracy occurs when $L$ additionally coincides with the $k$-axis (Figure $7(\mathrm{~b})$ ), so that $\theta_{1}= \pm \pi / 2, \theta_{2}=0$. This special case, in which all eigenvalues are geometrically double, was studied in detail by Groves [10] and Haragus \& Kirchgässner [13]. A degeneracy analogous to that shown in Figure 7(a) also occurs in cases $\mathrm{II}_{\mathrm{b}}$ and $\mathrm{II}_{\mathrm{c}}$ whenever $L$ passes through one of the points of intersection of $K_{1}$ with $C_{\mathrm{dr}}$ and $K_{-1}$ with $C_{\mathrm{dr}}$; a new geometrically double zero eigenvalue is created. In general values of $\theta_{1}$ for which the $K_{0}$ coordinates of two intersections of $\left\{K_{n}\right\}$ and $C_{\mathrm{dr}}$ in the $\left(K_{0}, L\right)$-coordinate system coincide lead to plus-minus pairs of geometrically double purely imaginary eigenvalues.

Decreasing $\nu$ further, one finds in cases $\mathrm{II}_{\mathrm{b}}, \mathrm{III}_{\mathrm{a}}$ and $\mathrm{III}_{\mathrm{c}}$ that there is a second critical value of $\nu$ at which $K_{1}$ and $K_{-1}$ become tangent to $C_{\mathrm{dr}}$; the resulting bifurcation scenario does not 

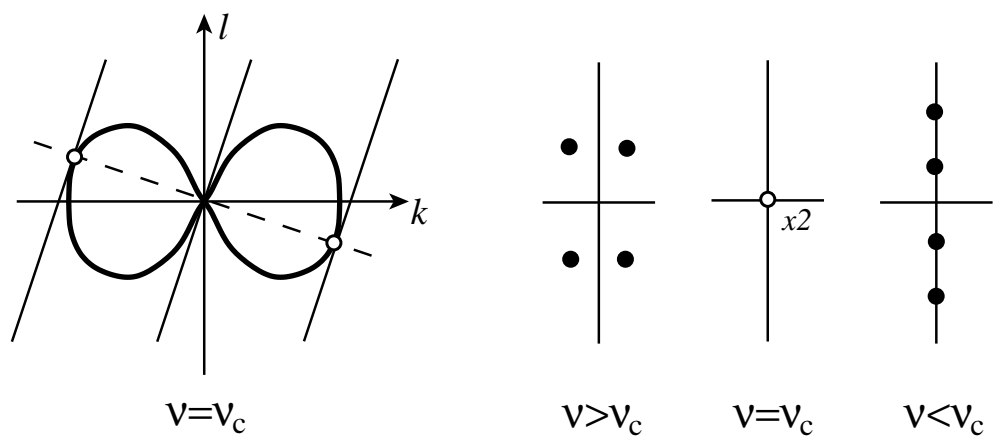

(a) A degenerate version of case $I I_{\mathrm{a}}$ in which the line $L$ passes through the points of intersection of $K_{1}$ with $C_{\mathrm{dr}}$ and $K_{-1}$ with $C_{\mathrm{dr}}$ at criticality: four complex eigenvalues become purely imaginary by passing through the origin.
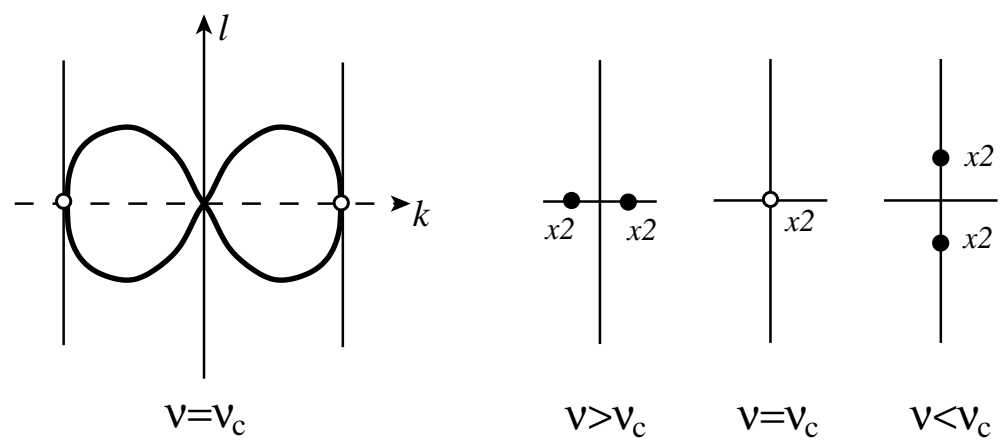

(b) A further degeneracy arises in case (a) above when the line $L$ coincides with the $k$-axis $\left(\theta_{1}= \pm \pi / 2, \theta_{2}=0\right)$ : two geometrically double real eigenvalues become purely imaginary by passing through the origin.

Figure 7: Degenerate versions of case $I I_{\mathrm{a}}$. The geometric multiplicity of the eigenvalues is shown; those depicted as solid dots are semisimple and those depicted as hollow dots have corresponding eigenvectors with Jordan chains of length 2.

involve $K_{n}$ for $|n| \geq 2$ provided that the second critical value of $\nu$ is greater than $\nu_{\mathrm{c}} / 2$. This situation is illustrated in Figure 8(a) for case $\mathrm{III}_{\mathrm{a}}$, where the number of purely imaginary eigenvalues decreases from four to zero in a Hamiltonian-Hopf bifurcation, and in Figure 8(b) for case $\mathrm{III}_{c}$, where the number of purely imaginary eigenvalues increases from eight to twelve in a Hamiltonian $\left(i \omega_{0}\right)^{2} \mathrm{i} \omega_{1} \mathrm{i} \omega_{2} \mathrm{i} \omega_{3} \mathrm{i} \omega_{4}$ resonance. Notice that the two tangencies in case $\mathrm{III}_{\mathrm{c}}$ occur simultaneously at $\nu=\nu_{\mathrm{c}}$ whenever $K_{0}$ coincides with the $k$-axis (Figure 8(c)); at criticality we have four simple purely imaginary eigenvalues accompanied by two pairs of geometrically simple eigenvalues whose eigenvectors (in modes \pm 1 ) have Jordan chains of length 2 (a Hamiltonian $\left(\mathrm{i} \omega_{0}\right)^{2}\left(\mathrm{i} \omega_{1}\right)^{2} \mathrm{i} \omega_{2} \mathrm{i} \omega_{3}$ resonance). Finally, a degenerate version of this bifurcation scenario occurs when $L$ additionally coincides with the $\ell$-axis (Figure $8(\mathrm{~d})$ ), so that $\theta_{1}=0, \theta_{2}= \pm \pi / 2$. This special case, in which all eigenvalues are geometrically double, was studied in detail by Groves \& Mielke [11]. (The case $\mathrm{II}_{\mathrm{b}}$ is analogous to case $\mathrm{III}_{\mathrm{c}}$; there are merely two fewer purely imaginary eigenvalues.) The above discussion relates to the 'nondegenerate' case when $C_{\mathrm{dr}}^{+}$is concave; the presence of points of inflection leads to further values of $\nu$ at which $K_{1}$ and $K_{-1}$ are tangent to $C_{\mathrm{dr}}$ and hence to further bifurcation scenarios which are catalogued similarly. 

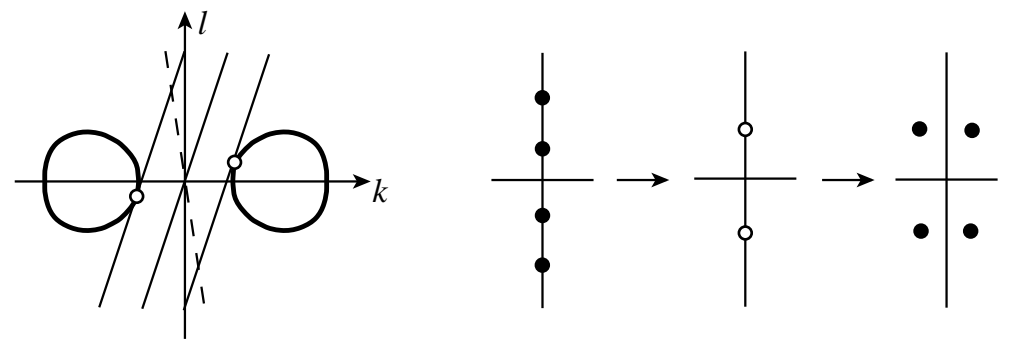

(a) The Hamiltonian-Hopf bifurcation in case $I_{\mathrm{a}}$ may be followed by a further HamiltonianHopf bifurcation as $\nu$ is decreased.

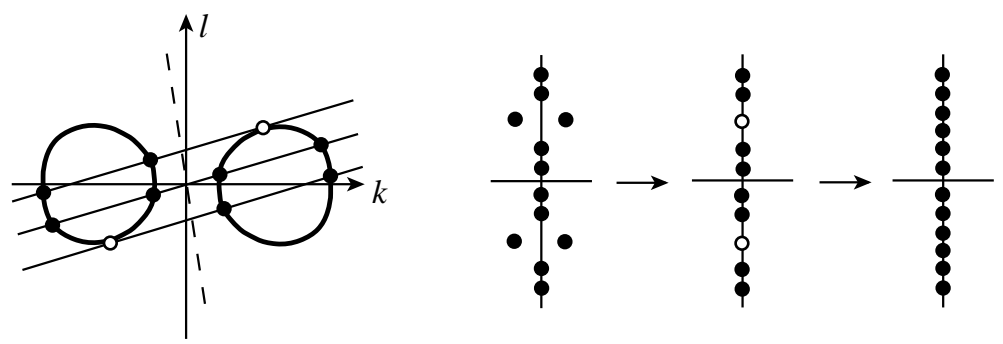

(b) The Hamiltonian $\left(\mathrm{i} \omega_{0}\right)^{2} \mathrm{i} \omega_{1} \mathrm{i} \omega_{2}$ resonance in case $I I I_{\mathrm{c}}$ may be followed by a Hamiltonian $\left(\mathrm{i} \omega_{0}\right)^{2} \mathrm{i} \omega_{1} \mathrm{i} \omega_{2} \mathrm{i} \omega_{3} \mathrm{i} \omega_{4}$ resonance as $\nu$ is decreased.
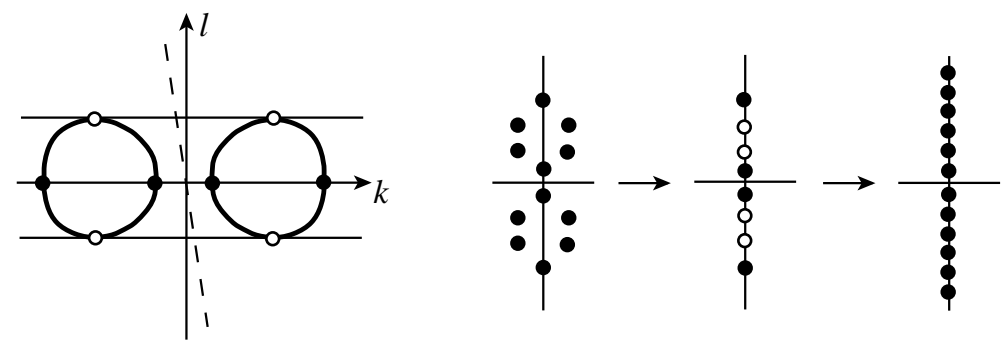

(c) The bifurcations in case $I I I_{\mathrm{c}}$ and (a) above occur simultaneously when $K_{0}$ coincides with the $k$-axis: the number of purely imaginary eigenvalues increases from four to twelve in a Hamiltonian $\left(\mathrm{i} \omega_{0}\right)^{2}\left(\mathrm{i} \omega_{1}\right)^{2} \mathrm{i} \omega_{2} \mathrm{i} \omega_{3}$ resonance.
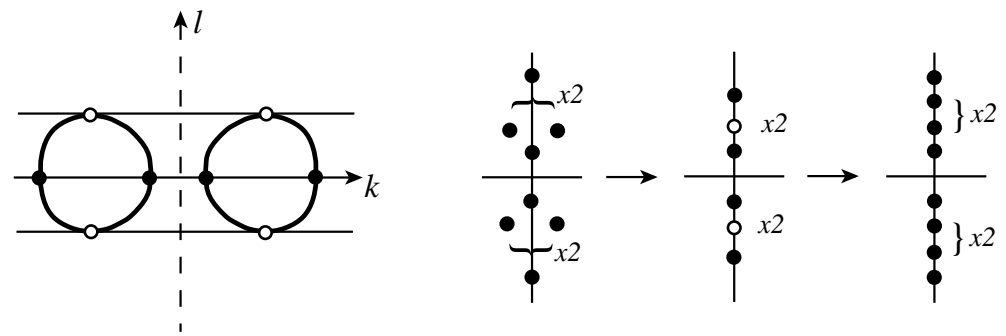

(d) A denegerate case of $(c)$ above occurs when in addition $L$ coincides with the $\ell$-axis $\left(\theta_{1}=0\right.$, $\left.\theta_{2}= \pm \pi / 2\right)$ : two geometrically double complex eigenvalues become purely imaginary without passing through the origin.

Figure 8: Further bifurcation scenarios in region III. The geometric multiplicity of the eigenvalues is shown; those depicted as solid dots are semisimple and those depicted as hollow dots have corresponding eigenvectors with Jordan chains of length 2. 

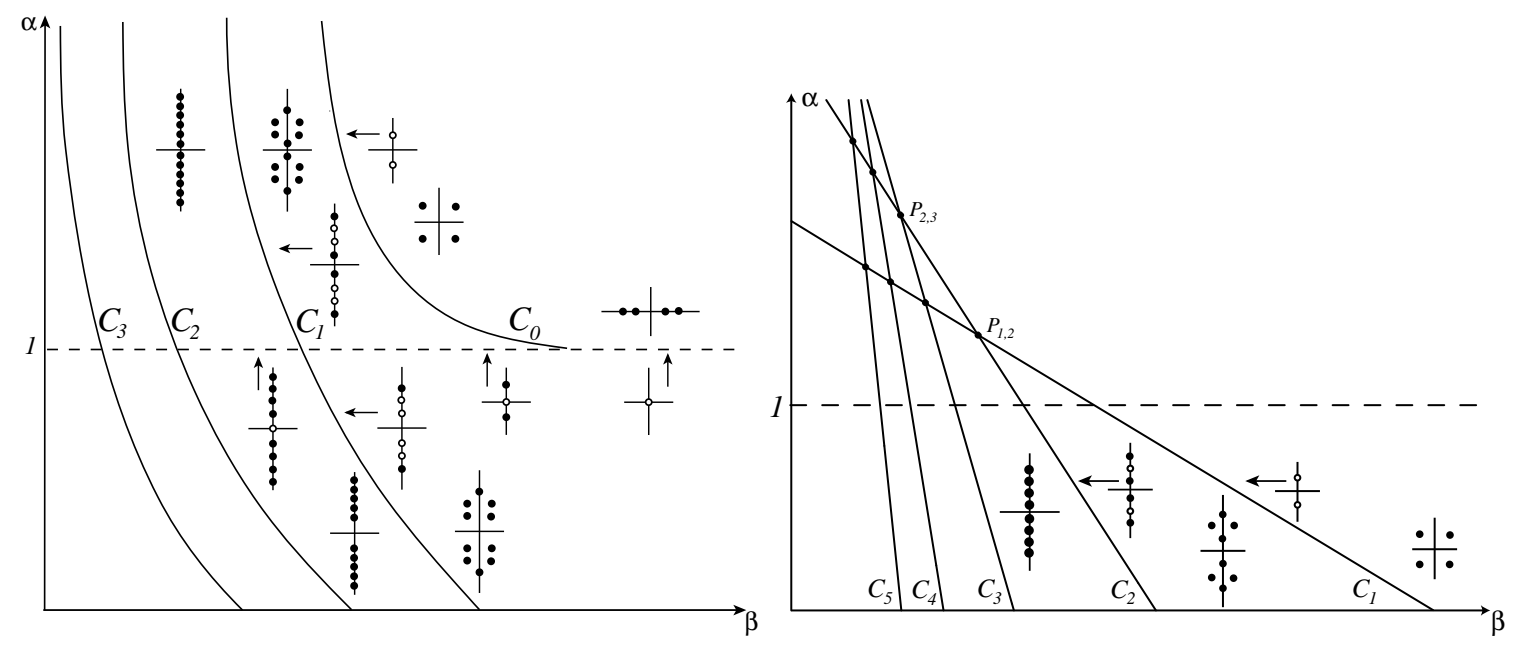

Figure 9: Bifurcation curves in $(\beta, \alpha)$ parameter space for $(a) \theta_{2}= \pm \pi / 2$ and $(b) \theta_{2}=0$.

Let us now turn to the complementary point of view in which one fixes the angles $\theta_{1}, \theta_{2}$ and frequency $\nu$ and looks for values of $(\beta, \alpha)$ at which the number of purely imaginary eigenvalues of $\mathcal{K}$ changes that is, values of $(\beta, \alpha)$ for which $\kappa$ is a double root of equation (39). One finds that $\mathcal{K}$ has a plus-minus pair of purely imaginary eigenvalues $i \kappa($ mode $n$ ) and $-i \kappa($ mode $-n$ ), each with an associated Jordan chain of length 2 , at each point of the curve $C_{n}$ in $(\beta, \alpha)$ parameter space given in parametric form by the formulae

$$
\begin{aligned}
\beta & =\frac{\operatorname{coth} \tilde{\Omega} \sin \theta_{2}\left(\kappa \sin \theta_{2}+n \nu \sin \theta_{1}\right)}{\tilde{\Omega}\left(\kappa+n \nu \cos \left(\theta_{1}-\theta_{2}\right)\right)}-\frac{\left(\operatorname{coth} \tilde{\Omega}+\tilde{\Omega} \operatorname{csch}^{2} \tilde{\Omega}\right)\left(\kappa \sin \theta_{2}+n \nu \sin \theta_{1}\right)^{2}}{2 \tilde{\Omega}^{3}}, \\
\alpha & =\frac{\left(3 \operatorname{coth} \tilde{\Omega}+\tilde{\Omega} \operatorname{csch}^{2} \tilde{\Omega}\right)\left(\kappa \sin \theta_{2}+n \nu \sin \theta_{1}\right)^{2}}{2 \tilde{\Omega}}-\frac{\tilde{\Omega} \operatorname{coth} \tilde{\Omega} \sin \theta_{2}\left(\kappa \sin \theta_{2}+n \nu \sin \theta_{1}\right)}{\kappa+n \nu \cos \left(\theta_{1}-\theta_{2}\right)} ;
\end{aligned}
$$

clearly $C_{0}$ is the curve $\Gamma_{\theta_{2}}$ in Figure 5. Observe that $C_{n}$ is the locus in $(\beta, \alpha)$ parameter space of those parameter values at which $K_{n}$ and $K_{-n}$ are tangent to $C_{\mathrm{dr}}$ in the $(k, \ell)$ plane. It follows from the above discussion that, except in the exceptional cases $\theta_{2}=0$ and $\theta_{2}= \pm \pi / 2$, the curve $C_{n}$ has at least two branches and may self-intersect in those regions of $(\beta, \alpha)$ parameter space for which $C_{\mathrm{dr}}^{+}$is not convex; furthermore each pair $C_{n}$ and $C_{m}$ typically also intersect in several points. When $\theta_{2}= \pm \pi / 2$ each $C_{n}$ consists of one branch and the curves $C_{n}$ are well ordered in the $(\beta, \alpha)$ plane, $C_{n+1}$ lying to the left of $C_{n}$ (see Figure 9(a)). In the further special case $\theta_{1}=0$, $\theta_{2}= \pm \pi / 2$ the mode $n$ and mode $-n$ eigenvalues for $n \neq 0$ coincide to form geometrically double eigenvalues; this case was investigated in detail by Groves \& Mielke [11]. When $\theta_{2}=0$ each bifurcation clearly occurs at $\ell=0$ in the $(k, \ell)$-plane, so that $\kappa=-n \nu \cos \theta_{1}$ and i $\kappa$ is always a double root of equation (39). It follows that $C_{n}$ is the straight line

$$
\left(\alpha+\beta n^{2} \nu^{2} \sin ^{2} \theta_{1}\right) \tanh \left(n \nu \sin \theta_{1}\right)=n \nu \sin \theta_{1},
$$

which connects a point $\left(\beta_{n}, 0\right)$ on the $\beta$-axis with a point $\left(0, \alpha_{n}\right)$ on the $\alpha$-axis, where $\beta_{1}>\beta_{2}>$ $\ldots$ and $\alpha_{1}<\alpha_{2}<\ldots$, so that every pair of curves $\left(C_{n}, C_{m}\right), n<m$ intersect in precisely one point $P_{n m}$ (see Figure 9(b)). In the further special case $\theta_{1}= \pm \pi / 2, \theta_{2}=0$ the mode $n$ and mode $-n$ eigenvalues for $n \neq 0$ again coincide to form geometrically double eigenvalues; this case was investigated in detail by Groves [10] and Haragus \& Kirchgässner [13]. 


\section{Existence theories for oblique waves}

\subsection{Coordinates on the centre manifold}

We begin by choosing a symplectic basis for $\mathcal{X}_{1}$ consisting of generalised eigenvectors of $L$. The operator always has a zero eigenvalue with eigenvector $v_{0}=(0,0,1,0)^{\mathrm{T}}$. Direct calculations show that this eigenvector has a Jordan chain of length 2 if $\alpha_{0} \neq \sin ^{2} \theta_{2}$, of length 4 if $\alpha_{0}=$ $\sin ^{2} \theta_{2}, \beta_{0} \neq \sin ^{2} \theta_{2} / 3$ and of length 6 if $\alpha_{0}=\sin ^{2} \theta_{2}, \beta_{0}=\sin ^{2} \theta_{2} / 3$. Starting from this Jordan chain, one can find a basis $\left\{e_{1}, \ldots, e_{n}, f_{1}, \ldots, f_{n}\right\}$ for $E_{0}$ consisting of generalised eigenvectors of $L_{s}$ such that $\Omega\left(e_{i}, f_{i}\right)=1$ and the symplectic product of any other combination of basis vectors is zero. Here $E_{0}$ is the generalised eigenspace corresponding to the zero eigenvalue and $n$ is 1,2 or 3 as appropriate. Notice that it is always possible to choose either $e_{1}$ or $f_{1}$ to be a scalar multiple of $v_{0}$. The real coordinates $q_{i}, p_{i}$ in the $e_{i}$ and $f_{i}$ directions are canonical coordinates for $E_{0}$ and the action of the reverser $\mathcal{R}$ on this generalised eigenspace is given by either $(q, p) \mapsto(-q, p)$ or $(q, p) \mapsto(q,-p)$.

Any other purely imaginary mode 0 eigenvalues occur in pairs $\pm \mathrm{i} q$ and there is an eigenvector

$$
e_{q}^{0}=\left(\begin{array}{c}
\frac{\mathrm{i} \sinh \mathrm{q}}{\sin \theta_{2}} \\
\cosh q \sin \theta_{2}-\sin \theta_{2} \frac{\sinh q}{q}-\frac{\beta q \sinh q}{\sin \theta_{2}} \\
\cosh q y \\
\text { iq } \cosh q y-\text { i sinh } q
\end{array}\right)
$$

such that $L e_{q}^{0}=\mathrm{i} q e_{q}^{0}, L \bar{e}_{q}^{0}=-\mathrm{i} q \bar{e}_{q}^{0}$. There is one such pair of eigenvalues when $\alpha_{0}<\sin ^{2} \theta_{2}$, and two pairs $\pm \mathrm{i} q_{1}, \pm \mathrm{i} q_{2}$ exist when $\alpha_{0}>\sin ^{2} \theta_{2}$ and $\left(\beta_{0}, \alpha_{0}\right)$ lies to the left of the curve $\Gamma_{\theta_{2}}$ (see Section 3.2 above). In the latter case the two pairs of purely imaginary eigenvalues have opposite Krein signature since they are created in a Hamiltonian-Hopf bifurcation at $\Gamma_{\theta_{2}}$ (a direct calculation shows that the pair of lesser modulus has the negative Krein signature). When $\left(\beta_{0}, \alpha_{0}\right) \notin \Gamma_{\theta_{2}}$ one can normalise $e_{q}^{0}$ in such a way that $\Omega\left(e_{q}^{0}, \bar{e}_{q}^{0}\right)= \pm \mathrm{i}$, so that $\left\{e_{q}^{0}, \bar{e}_{q}^{0}\right\}$ is a symplectic basis for $E_{\mathrm{i} q} \oplus E_{-\mathrm{i} q}$. Canonical coordinates for $E_{\mathrm{i} q} \oplus E_{-\mathrm{i} q}$ are given by the complex coordinates $C, \bar{C}$ in the $e_{q}^{0}, \bar{e}_{q}^{0}$ directions, and the action of the reverser $\mathcal{R}$ on $E_{\mathrm{i} q} \oplus E_{-\mathrm{i} q}$ is $C \mapsto \bar{C}$. When $\left(\beta_{0}, \alpha_{0}\right) \in \Gamma_{\theta_{2}}$ there is a generalised eigenvector

$$
f_{q}^{0}=\left(\begin{array}{c}
\frac{\cosh q}{\sin \theta_{2}} \\
\frac{\mathrm{i}}{q} \cosh q\left(\frac{\beta q^{2}}{\sin \theta_{2}}+\sin \theta_{2}\right)+\frac{\mathrm{i}}{q^{2}} \sinh q\left(\frac{\beta q^{2}}{\sin \theta_{2}}-\left(1+q^{2}\right) \sin \theta_{2}\right) \\
-\mathrm{i} y \sinh q y \\
-\cosh q+\cosh q y+q y \sinh q y
\end{array}\right)
$$

such that $(L-\mathrm{i} q I) f_{q}^{0}=e_{q}^{0},(L+\mathrm{i} q I) \bar{f}_{q}^{0}=\bar{e}_{q}^{0}$. After normalising $e_{q}^{0}$ and modifying $f_{q}^{0}$ by the addition of a suitable multiple of $e_{q}^{0}$ if necessary, one finds that $\left\{e_{q}^{0}, f_{q}^{0}, \bar{e}_{q}^{0}, \bar{f}_{q}^{0}\right\}$ is a basis for $E_{\mathrm{i} q} \oplus E_{-\mathrm{i} q}$ such that $\Omega\left(e_{q}^{0}, \bar{f}_{q}^{0}\right)=1, \Omega\left(f_{q}^{0}, \bar{e}_{q}^{0}\right)=-1$ and the symplectic product of any other combination of basis vectors is zero. The complex coordinates $A, B$ in the $e$ and $f$ directions are canonical coordinates for $E_{\mathrm{i} q} \oplus E_{-\mathrm{i} q}$, the action of the reverser $\mathcal{R}$ on this space is $(A, B) \mapsto$ $(\bar{A},-\bar{B})$. 
Purely imaginary eigenvalues in higher Fourier modes occur in pairs $\pm \mathrm{i} s$, where

$$
L e_{s}^{n} \mathrm{e}^{\mathrm{i} n z}=\mathrm{i} s e_{s}^{n} \mathrm{e}^{\mathrm{i} n z}, \quad L \bar{e}_{s}^{n} \mathrm{e}^{-\mathrm{i} n z}=-\mathrm{i} s \bar{e}_{s}^{n} \mathrm{e}^{-\mathrm{i} n z} .
$$

Each such pair $\pm \mathrm{i} s_{1}$ is typically created together with a partner pair $\pm \mathrm{i} s_{2}$ of opposite Krein signature in a $1:-1$ resonance at a point of the curve $C_{n}$ (see Section 3.3 above). Of course for fixed $\alpha_{0}, \beta_{0}, \nu, \theta_{2}$ there are special values of $\theta_{1}$ for which $s_{1}=s_{2}$ (a semisimple resonance) or one of $s_{1}, s_{2}$ vanishes, the Krein signature of $\pm \mathrm{i} s_{1}$ or $\pm \mathrm{i} s_{2}$ thereby changing in a semisimple resonance. Furthermore the pair $\pm \mathrm{i} \kappa$ of purely imaginary eigenvalues in the $1:-1$ resonance giving rise to $\pm \mathrm{i} s_{1}, \pm \mathrm{i} s_{2}$ may coincide in the origin (cf. Figure 7(a)), be geometrically double (cf. Figure 8(d)) or indeed both (cf. Figure 7(b)). Away from $1:-1$ resonances we can normalise the eigenvectors corresponding to the eigenvalues $\pm \mathrm{i} s$ in such a way that $\Omega\left(e_{s}^{n}, \bar{e}_{s}^{n}\right)= \pm \mathrm{i}$, so that $\left\{e_{s}^{n} \mathrm{e}^{\mathrm{i} n z}, \bar{e}_{s}^{n} \mathrm{e}^{-\mathrm{i} n z}\right\}$ is a symplectic basis for $E_{\mathrm{i} s} \oplus E_{-\mathrm{i} s}$; canonical coordinates are given by the complex coordinates $C_{n}, \bar{C}_{n}$ in the directions of the symplectic basis, and the action of the reverser $\mathcal{R}$ is $C_{n} \mapsto \bar{C}_{n}$. At a $1:-1$ resonance involving the eigenvalues $\pm \mathrm{i} s$ we find that there are generalised eigenvectors such that

$$
(L-\mathrm{i} s I) f_{s}^{n} \mathrm{e}^{\mathrm{i} n z}=e_{s}^{n} \mathrm{e}^{\mathrm{i} n z}, \quad(L+\mathrm{i} s I) \bar{f}_{s}^{n} \mathrm{e}^{-\mathrm{i} n z}=\bar{e}_{s}^{n} \mathrm{e}^{-\mathrm{i} n z} .
$$

The usual normalisation yields $\Omega\left(e_{s}^{n}, \bar{f}_{s}^{n}\right)=1, \Omega\left(f_{s}^{n}, \bar{e}_{s}^{n}\right)=-1$ and the symplectic product of any other combination of basis vectors is zero. The complex coordinates $A_{n}, B_{n}$ in the $e_{s}^{n}$ and $f_{s}^{n}$ directions are canonical coordinates for $E_{\mathrm{i} s} \oplus E_{-\mathrm{i} s}$ and the action of the reverser $\mathcal{R}$ on this space is $\left(A_{n}, B_{n}\right) \mapsto\left(\bar{A}_{n},-\bar{B}_{n}\right)$.

Let us now turn briefly to the special cases $\theta_{1}=0, \theta_{2}= \pm \pi / 2$ and $\theta_{1}= \pm \pi / 2, \theta_{2}=0$ in which all eigenvalues are geometrically double and Hamilton's equations have the additional symmetry $\mathcal{S}_{1}$ or $\mathcal{S}_{2}$. Focusing upon pairs \pm is of purely imaginary eigenvalues not involved in $1:-1$ resonances, we find in the former case that

$$
\begin{array}{ll}
L e_{s}^{n} \cos n z=\mathrm{i} s e_{s}^{n} \cos n z, & L \bar{e}_{s}^{n} \cos n z=-\mathrm{i} s \bar{e}_{s}^{n} \cos n z, \\
L e_{s}^{n} \sin n z=\mathrm{i} s e_{s}^{n} \sin n z, & L \bar{e}_{s}^{n} \sin n z=-\mathrm{i} s \bar{e}_{s}^{n} \sin n z
\end{array}
$$

So that $\left\{e_{s}^{n} \cos n z, \bar{e}_{s}^{n} \cos n z, e_{s}^{n} \sin n z, \bar{e}_{s}^{n} \sin n z\right\}$ is a symplectic basis for $E_{\mathrm{i} s} \oplus E_{-\mathrm{i} s}$; canonical coordinates are given by the complex coordinates $C^{1}, \bar{C}^{1}, C^{2}, \bar{C}^{2}$ in the directions of the symplectic basis, and the actions of the reverser $\mathcal{R}$ and reflector $\mathcal{S}_{1}$ are respectively $\left(C^{1}, C^{2}\right) \mapsto$ $\left(\bar{C}^{1}, \bar{C}^{2}\right)$ and $\left(C_{s}^{1}, C_{s}^{2}\right) \mapsto\left(C_{s}^{1},-C_{s}^{2}\right)$. In the latter case we find that $L v_{+}^{s, n}=\mathrm{i} s v_{+}^{s, n}, L \bar{v}_{+}^{s, n}=$ $-\mathrm{i} s \bar{v}_{+}^{s, n}, \mathcal{S}_{2} v_{+}^{s, n}=v_{+}^{s, n}$ and $L v_{-}^{s, n}=\mathrm{i} s v_{-}^{s, n}, L \bar{v}_{-}^{s, n}=-\mathrm{i} \bar{s} v_{-}^{s, n}, \mathcal{S}_{2} v_{-}^{s, n}=-v_{-}^{s, n}$, and these eigenvectors can be normalised so that

$$
\Omega\left(v_{+}^{s, n}, \bar{v}_{+}^{s, n}\right)=\Omega\left(v_{-}^{s, n}, \bar{v}_{-}^{s, n}\right)=-\mathrm{i}
$$

so that both pairs is of eigenvalues have positive Krein signature (see Section 4.2 below). Clearly one finds that $\left(v_{+}^{s, n}, \bar{v}_{+}^{s, n}, v_{-}^{s, n}, \bar{v}_{-}^{s, n}\right)$ is a symplectic basis for $E_{\mathrm{i} s} \oplus E_{-\mathrm{i} s}$; canonical coordinates are given by the complex coordinates $C^{1}, \bar{C}^{1}, C^{2}, \bar{C}^{2}$ in the directions of the symplectic basis, and the actions of the reverser $\mathcal{R}$ and reflector $\mathcal{S}_{2}$ are respectively $\left(C^{1}, C^{2}\right) \mapsto\left(\bar{C}^{1}, \bar{C}^{2}\right)$ and $\left(C^{1}, C^{2}\right) \mapsto\left(C^{1},-C^{2}\right)$. (It is no contradiction that two pairs $\pm \mathrm{i} s_{1}, \pm \mathrm{i} s_{2}$ of imaginary eigenvalues with opposite Krein signature are created in a $1:-1$ resonance at points of the line $C_{n}$ 
when $\theta_{2}=0, \theta_{1} \neq \pm \pi / 2$. Upon varying $\theta_{1}$ to $\pm \pi / 2$, one finds that the two purely imaginary eigenvalues of lesser magnitude pass through the origin in a semisimple resonance, thus changing their Krein signature, and then increase their magnitude to coincide with the other pair. This behaviour is readily predicted by varying the angle $\theta_{1}$ in Figure $7(\mathrm{~b})$ for $\nu \leq \nu_{\mathrm{c}}$.)

Observe that $\Omega$ is the canonical symplectic 2 -form $\Upsilon$ in the coordinate system introduced above, and according to the remarks at the end of Section 2.3 it is, after a near identity change of variables, convenient to study the centre manifold in this coordinate system. The subspace

$$
\mathcal{T}=\left\{A_{n}=B_{n}=C_{n}=0\right\}
$$

of $\mathcal{X}_{1}$ is important in the following theory: it consists of $z$-independent vectors and is an invariant subspace for the reduced dynamics. Note also that the action $\Phi \mapsto \Phi+c$ of the translation group on $\mathcal{X}_{1}$ is given by either $q_{1} \mapsto q_{1}+c$ or $p_{1} \mapsto p_{1}+c$. The centre-manifold reduction procedure preserves reversibility and symmetries, so that the action of the reverser $\mathcal{R}$ upon the coordinates for $\tilde{W}_{1}$ introduced above correctly describes the reversibility of $\left(M_{C}^{\lambda}, \Upsilon, \tilde{H}^{\lambda}\right)$, for which $\mathcal{T}$ is an invariant subspace. The translation invariance in $\Phi$ is reflected in the fact that either $q_{1}$ or $p_{1}$ is always cyclic; the conjugate variable is a conserved quantity (which will be set to zero in the following analysis), and the dimension of the system of equations can always be reduced by two.

It remains to find solutions of the reduced equations on the centre manifold which correspond to three-dimensional travelling water waves of interest. This task is undertaken in Sections 4.2 and 4.3 below, where we take advantage of the Hamiltonian structure of the reduced equations to find solutions by applying available existence theories for general classes of Hamiltonian systems.

\subsection{Periodic waves}

A $2 \pi / \kappa$-periodic solution of the reduced equations on the centre manifold corresponds to a doubly periodic travelling water wave which is $2 \pi / \kappa$-periodic in $x$ and $2 \pi / \nu$-periodic in $z$. Such solutions may be found by applying an appropriate version of the Lyapunov centre theorem to the reduced equations, which constitute Hamilton's equations for a finite-dimensional Hamiltonian system. The number and character of these solutions therefore depends crucially upon the purely imaginary eigenvalues of the original hydrodynamic problem.

Let us begin by examining $z$-independent solutions of the water-wave problem, so that the reduction procedure involves only mode 0 quantities and the centre manifold lies in the subspace $\mathcal{T}$. A $2 \pi / \kappa$-periodic solution of the reduced equations in this case corresponds to a travelling water wave which is $2 \pi / \kappa$-periodic in $x$ and independent of $z$; a wave of this kind is sketched in Figure 3. The behaviour of the mode 0 eigenvalues is described comprehensively in Section 3.2 above; in particular Figure 4 shows that the reduced Hamiltonian system has one pair $\pm \mathrm{i} q$

of purely imaginary eigenvalues when $\alpha<\sin ^{2} \theta_{2}$ and two pairs $\pm \mathrm{i} q_{1}, \pm \mathrm{i} q_{2}$ when $\alpha>\sin ^{2} \theta_{2}$ and $(\beta, \alpha)$ lies to the left of the curve $\Gamma_{\theta_{2}}$. A direct calculation shows that $q_{2} / q_{1}$ is equal to the positive integer $k \geq 2$ when $(\beta, \alpha)$ lies on the curve $R_{k}$ given in parametric form by

$$
\begin{aligned}
& \beta=\frac{\sin ^{2} \theta_{2}}{\left(1-k^{2}\right) q \tanh q}-\frac{k \sin ^{2} \theta_{2}}{q\left(1-k^{2}\right) \tanh k q}, \\
& \alpha=-\frac{k^{2} q \sin ^{2} \theta_{2}}{\left(1-k^{2}\right) \tanh q}+\frac{q k \sin ^{2} \theta_{2}}{\left(1-k^{2}\right) \tanh k q} ;
\end{aligned}
$$




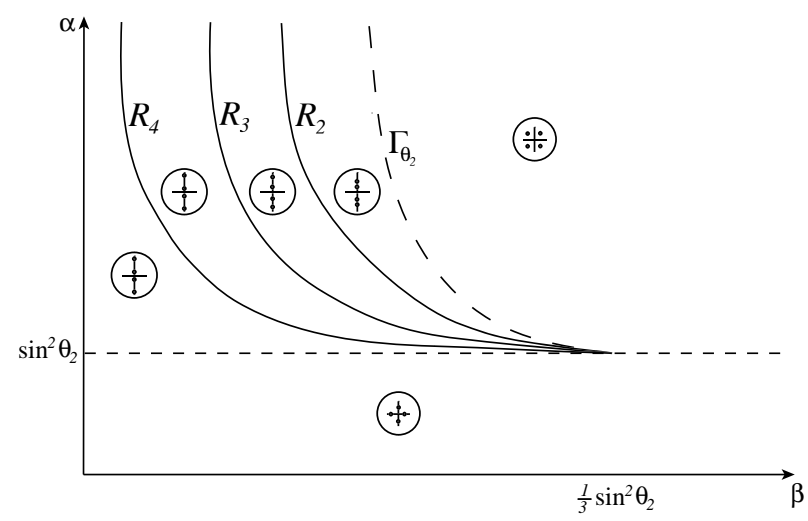

Figure 10: The two pairs of purely imaginary mode 0 eigenvalues to the left of $\Gamma_{\theta_{2}}$ and above $\left\{\alpha=\sin ^{2} \theta_{2}\right\}$ lie in $-1: k$ resonance at points of the curve $R_{k}$.

these curves are sketched in Figure 10. The following result is obtained by a direct application of the classical nonresonant Lyapunov centre theorem (e.g. see Ambrosetti \& Prodi [2, Chapter 7, Theorem 3.4]).

\section{Theorem 4}

(i) Suppose that $\alpha<\sin ^{2} \theta_{2}$. The reduced equations on the centre manifold admit a periodic solution on the energy surface $\left\{\widetilde{H}_{\mathrm{C}}=\epsilon\right\}$ for each sufficiently small value of $\epsilon>0$.

(ii) Suppose that $\alpha>\sin ^{2} \theta_{2}$, that $(\beta, \alpha)$ lies to the left of $\Gamma_{\theta_{2}}$ and that $(\beta, \alpha) \notin R_{k}$ for any $k$. The reduced equations on the centre manifold admit two geometrically distinct periodic orbits on the energy surface $\left\{\widetilde{H}_{\mathrm{C}}=\epsilon\right\}$ for each sufficiently small value of $\epsilon>0$.

The periodic solutions identified above correspond to travelling water waves which are periodic in $x$ and independent of $z$.

The eigenvalues $\pm \mathrm{i} s_{1}, \pm \mathrm{i} s_{2}$ are created in a $1:-1$ resonance at points of the curve $\Gamma_{\theta_{2}}$ and therefore have opposite Krein signature. A direct calculation shows that the Krein signature of the pair of lesser magnitude is negative, so that a $-1: k$ eigenvalue resonance takes place at points of the curve $R_{k}$. This situation, in which the quadratic part of the Hamiltonian is not positive definite, was examined by Schmidt [30] and Duistermaat [9]. The Schmidt-Duistermaat theory is an existence theory for small-amplitude periodic solutions of two-degree-of-freedom Hamiltonian systems with two pairs of imaginary eigenvalues in $1: k$ or $1:-k$ resonance, and the resonant eigenvalues $\pm \mathrm{i} q, \pm \mathrm{i} k q$ are also allowed to depend upon a detuning parameter $\mu$ which takes them out of resonance for $\mu \neq 0$. The theory is based upon a Lyapunov-Schmidt reduction (Schmidt) and singularity theory (Duistermaat), and is in essence a persistence result for periodic solutions of the fourth-order Birkhoff normal form associated with the resonance.

Turning to the present water-wave problem, let us introduce a detuning parameter by choosing $\left(\beta_{0}, \alpha_{0}\right) \in R_{k}$ and writing $\alpha=\alpha_{0}+\mu$ and carry out the centre-manifold reduction procedure using the coordinate system described in Section 4.1 above. The Birkhoff normal-form theory 

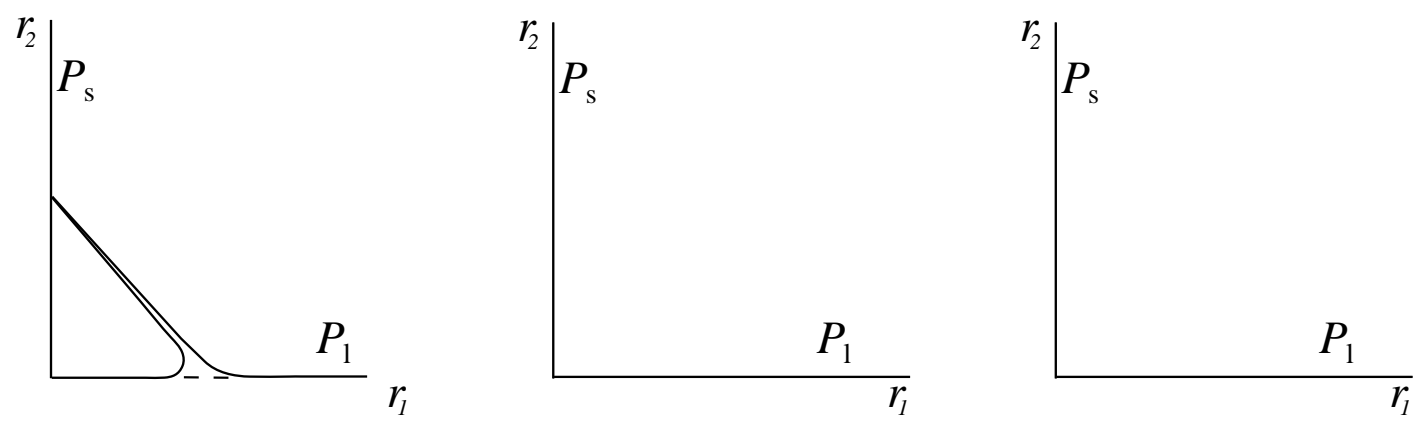

(a) The $-1: 4$ resonance: $\mu>0$ (left), $\mu=0$ (centre), $\mu<0$ (right).
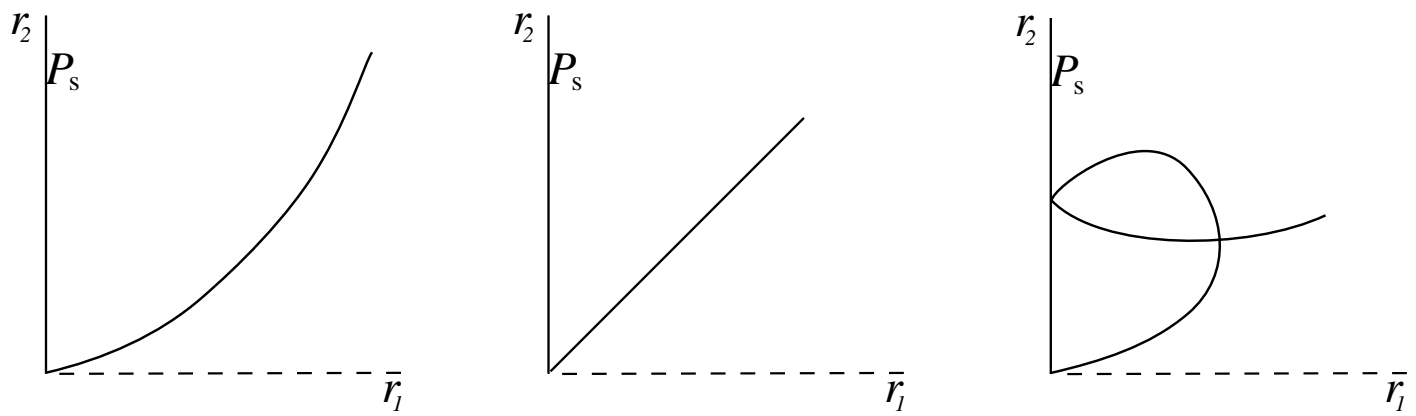

(b) The $-1: 3$ resonance: $\mu>0$ (left), $\mu=0$ (centre), $\mu<0$ (right).
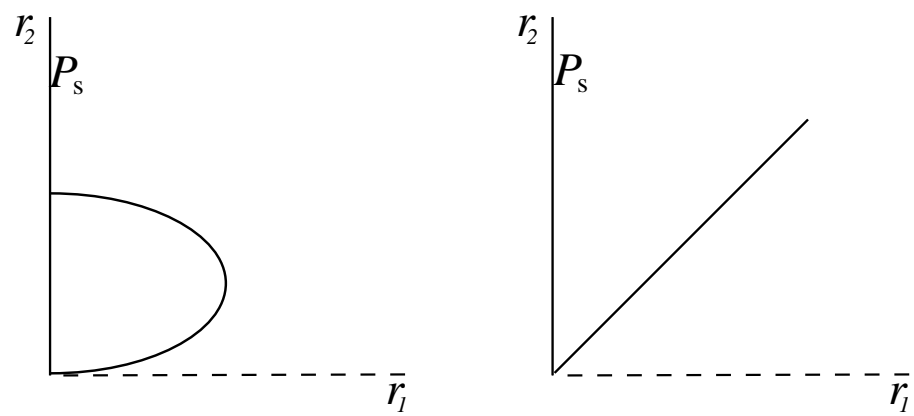

(c) The $-1: 2$ resonance: $\mu \neq 0$ (left), $\mu=0$ (right).

Figure 11: Schmidt-Duistermaat bifurcation diagrams for $\left(\beta_{0}, \alpha_{0}\right) \in R_{k}, \alpha=\alpha_{0}+\mu$; each point on a solid line represents a periodic solution. The symbols $\mathcal{P}_{\mathrm{s}}, \mathcal{P}_{1}$ denote the short and long period waves associated with the (nonresonant) Lyapunov centre theorem and $r_{1}, r_{2}$ are polar coordinates along the (nonresonant) Lyapunov manifolds. 
states that there is a near-identity, analytic, symplectic change of variables which transforms the Hamiltonian in the reduced Hamiltonian system into

$$
\left.H=-q\left|C_{1}\right|^{2}+k q\left|C_{2}\right|^{2}+\frac{1}{2} c_{1}\left|C_{1}\right|^{4}+c_{2}\left|C_{1}\right|^{2}\left|C_{2}\right|^{2}+\frac{1}{2} c_{3}\left|C_{2}\right|^{4}+\mathcal{O}\left(|\mu|\left|\left(C_{1}, C_{2}\right)\right|^{2}\right)+\left.\mathcal{O}\left(\mid C_{1}, C_{2}\right)\right|^{5}\right)
$$

for the $-1: k$ resonance, $k \geq 4$, into

$$
\begin{gathered}
H=-q\left|C_{1}\right|^{2}+3 q\left|C_{2}\right|^{2}+c_{4} C_{1}^{3} \bar{C}_{2}+\bar{c}_{4} \bar{C}_{1}^{3} C_{2}+\frac{1}{2} c_{1}\left|C_{1}\right|^{4}+c_{2}\left|C_{1}\right|^{2}\left|C_{2}\right|^{2}+\frac{1}{2} c_{3}\left|C_{2}\right|^{4} \\
\left.+\mathcal{O}\left(|\mu|\left|\left(C_{1}, C_{2}\right)\right|^{2}\right)+\left.\mathcal{O}\left(\mid C_{1}, C_{2}\right)\right|^{5}\right)
\end{gathered}
$$

for the $-1: 3$ resonance and into

$$
\begin{gathered}
H=-q\left|C_{1}\right|^{2}+2 q\left|C_{2}\right|^{2}+c_{4} C_{1}^{2} \bar{C}_{2}+\bar{c}_{4} \bar{C}_{1}^{2} C_{2}+\frac{1}{2} c_{1}\left|C_{1}\right|^{4}+c_{2}\left|C_{1}\right|^{2}\left|C_{2}\right|^{2}+\frac{1}{2} c_{3}\left|C_{2}\right|^{4} \\
\left.+\mathcal{O}\left(|\mu|\left|\left(C_{1}, C_{2}\right)\right|^{2}\right)+\left.\mathcal{O}\left(\mid C_{1}, C_{2}\right)\right|^{5}\right) .
\end{gathered}
$$

for the $-1: 2$ resonance. The hypotheses in the Schmidt-Duistermaat theory take the form of conditions on the real coefficients $c_{1}, c_{2}, c_{3}$; notice however that it is necessary to convert the $-1: k$ resonances here into $1:-k$ resonances to apply the theory directly (for example using the transformation $z_{1}=\mathrm{i} \bar{C}_{1}, z_{2}=\mathrm{i} \bar{C}_{2}$, which is a symplectic change of variable with multiplier -1). The coefficients $c_{1}, c_{2}, c_{3}$ are efficiently calculated using a procedure explained in detail by Groves \& Mielke [11, Appendix B] and are stated in Appendix A of the present paper. The Schmidt-Duistermaat bifurcation diagrams for small-amplitude periodic solutions near the $-1: k$ resonances are given in Figure 11 . In the special case $\theta_{1}=0, \theta_{2}=\pi / 2$ (that is, two-dimensional travelling water waves) periodic waves associated with the $-1: 2$ resonance are referred to as 'Wilton ripples' (Wilton [33]) and have been investigated using a LyapunovSchmidt reduction of a formulation of the two-dimensional travelling water-wave problem as an integral equation by Jones [18]; his results are recovered and extended in Figure 11(c). Of course the results presented above all apply to the two-dimensional problem and yield results which are also novel in this special case.

Let us now turn to the general case in which eigenvalues associated with higher Fourier modes are also involved. A procedure for identifying all purely imaginary eigenvalues is explained in detail in Section 3.3 above, where in particular a full catalogue of situations involving only mode 0 , mode 1 and mode -1 purely imaginary eigenvalues is given. In principle periodic solutions of the reduced equations, corresponding to doubly periodic travelling water waves, can be found by applying the Lyapunov centre theorem. There are, however, many choices of parameters for which eigenvalues are in resonance, and these resonances can involve eigenvalue pairs of equal or opposite Krein signatures. The following theorem is a representative application of the Lyapunov centre theorem to periodic solutions controlled by mode \pm 1 purely imaginary eigenvalues. It has the character of an 'inverse' result in which the fundamental domain of a doubly periodic surface wave is specified and values of the physical parameters $\alpha$ and $\beta$ are found which guarantee the existence of the desired wave, an example of which is sketched in Figure 3 . The parameters are selected so that $\pm i \kappa$ are mode \pm 1 eigenvalues which are not in nonsemisimple resonance with any other mode \pm 1 eigenvalues or in semisimple resonance with eigenvalues in any of the other Fourier modes. 
Theorem 5 Choose angles $\theta_{1}, \theta_{2}$ and frequencies $\kappa$ and $\nu$ in respectively $x$ and $z$. For any pair $(\beta, \alpha)$ on the line

$$
\alpha+\widetilde{\gamma}^{2} \beta=\frac{\left(\sin \theta_{2} \kappa+\sin \theta_{1} \nu\right)^{2}}{\widetilde{\gamma} \tanh \widetilde{\gamma}},
$$

where $\widetilde{\gamma}^{2}=\kappa^{2}+\nu^{2}+2 \cos \left(\theta_{1}-\theta_{2}\right) \nu \kappa$, and which does not belong to the curve $C_{1}$ or any of the lines

$$
\alpha+\widetilde{\gamma}_{m, n}^{2} \beta=\frac{\left(m \sin \theta_{2} \kappa+n \sin \theta_{1} \nu\right)^{2}}{\widetilde{\gamma}_{m, n} \tanh \widetilde{\Omega}_{m, n}}, \quad(m, n) \in \mathbb{N}_{0} \times \mathbb{N}_{0} \backslash\{(1,1)\},
$$

where $\widetilde{\gamma}_{m, n}^{2}=m^{2} \kappa^{2}+n^{2} \nu^{2}+2 \cos \left(\theta_{1}-\theta_{2}\right) m n \nu \kappa$, the reduced equations on the centre manifold possess a periodic orbit on the energy surface $\left\{\widetilde{H}_{C}^{0}=\epsilon\right\}$ for each sufficiently small value of $\epsilon>0$. Each of these periodic orbits corresponds to a travelling water wave which is periodic in $x$ and $z$ with frequencies respectively near $\kappa$ and equal to $\nu$.

Craig \& Nicholls [5] have recently given results of this kind using a variational LyapunovSchmidt reduction of the equations for travelling water waves; Both approaches give existence theories for the 'hexagonal' wave patterns observed in nature and predicted on the basis of model equations (Hammack, Scheffner \& Segur [12]) (take $\left|\theta_{1}-\theta_{2}\right|=\pi / 3$ for a true hexagonal fundamental domain) and for the 'short-crested waves' whose fundamental domain is a 'symmetric diamond' (doubly periodic waves with equal periods) and whose existence was proved by Reeder \& Shinbrot [29].

We conclude this Section with an illustrative example of an application a version of the Lyapunov centre theorem for eigenvalues in resonance. Consider the case $\theta_{1}= \pm \pi / 2$ and $\theta_{2}=0$, where all eigenvalues are real or purely imaginary and always have double geometric multiplicity. In particular, the eigenspace corresponding to a nonzero purely imaginary eigenvalue i $q$ is spanned by the eigenvectors

$$
v_{+}^{s, n}=\left(\begin{array}{c}
\frac{\mathrm{i} \tilde{\gamma}}{n \nu} \sinh \tilde{\gamma} \cos n z \\
-\frac{\beta q \tilde{\gamma}}{n \nu} \sinh \tilde{\gamma} \cos n z \\
\mathrm{i} \cosh (\tilde{\gamma} y) \sin n z \\
-q \cosh (\tilde{\gamma} y) \sin n z
\end{array}\right), \quad v_{-}^{s, n}=\left(\begin{array}{c}
-\frac{\tilde{\gamma}}{n \nu} \sinh \tilde{\gamma} \sin n z \\
\mathrm{i} \tilde{\beta} \tilde{\gamma} \sinh \tilde{\gamma} \sin n z \\
n \nu \\
\cosh (\tilde{\gamma} y) \cos n z \\
\mathrm{i} q \cosh (\tilde{\gamma} y) \cos n z
\end{array}\right)
$$

which are respectively symmetric and antisymmetric with respect to the reflector $\mathcal{S}_{2}$, and a direct calculation shows that

$$
\Omega\left(\mathrm{i} v_{+}^{s, n}, \bar{v}_{+}^{s, n}\right)=\Omega\left(\mathrm{i} v_{-}^{s, n}, \bar{v}_{-}^{s, n}\right)=\frac{\pi q}{n^{2} \nu^{2} \tilde{\gamma}}\left(4 \beta \tilde{\gamma}^{3} \sinh ^{2} \tilde{\gamma}+n^{2} \nu^{2}(2 \tilde{\gamma}+\sinh 2 \tilde{\gamma})\right)>0
$$

It follows that for $(\beta, \alpha) \notin R_{k}$ for any $k$ all pairs of purely imaginary eigenvalues have positive Krein signature, so that the quadratic part of the reduced Hamiltonian is positive definite. We can therefore apply the resonant version of the Lyapunov centre theorem due to Weinstein [32] and further developed by Moser [26], which states that the nonresonance condition on the eigenvalues can be replaced by the requirement that the quadratic part of the Hamiltonian is positive-definite. The following result is obtained by applying the Weinstein-Moser theorem to the reduced Hamiltonian system and to its further reduction by the symmetry $\mathcal{S}_{2}$; in the latter case we recover the result given by Groves [10, Theorem 5] with the nonresonance condition removed. 
Theorem 6 Suppose that $\theta_{1}= \pm \pi / 2$ and $\theta_{2}=0$.

(i) Suppose that $\left(\beta_{0}, \alpha_{0}\right)$ lies below the line $C_{1}$, to the left of $C_{j}$ and to the right of $C_{j+1}$ for some $j \in \mathbb{N}$. The reduced equations on the centre manifold have $2 j$ geometrically distinct periodic orbits on the energy surface $\left\{\tilde{H}^{0}=\epsilon\right\}$ for each sufficiently small value of $\epsilon>0$ and $j$ of these orbits are invariant under $\mathcal{S}_{2}$.

(ii) Suppose that $\left(\beta_{0}, \alpha_{0}\right)$ lies above the line $C_{i}$ and below the line $C_{i+1}$ for some $i \in \mathbb{N}$, to the left of $C_{j}$ and to the right of $C_{j+1}$ for some $j \geq i$. The reduced equations on the centre manifold have $2 j$ geometrically distinct periodic orbits on the energy surface $\left\{\tilde{H}^{0}=\epsilon\right\}$ for each sufficiently small value of $\epsilon>0$ and $j$ of these orbits are invariant under $\mathcal{S}_{2}$.

The periodic solutions identified above correspond to doubly periodic travelling water waves whose fundamental domain is a rectangle aligned with the direction of propagation.

\subsection{Waves of infinite spatial extent}

Let us now turn to waves which have an aperiodic profile in the $x$ ('timelike') direction; in particular we discuss waves whose profile in this direction resembles a solitary or generalised solitary wave. We again begin by briefly examining $z$-independent solutions of the water-wave problem, so that the reduction procedure involves only mode 0 quantities and the centre manifold lies in the subspace $\mathcal{T}$. The behaviour of the mode 0 eigenvalues is described comprehensively in Section 3.2 above; in particular Figure 4 shows that they undergo a $0^{2}$ resonance along the line segment $\left\{(\beta, \alpha): \alpha=\sin ^{2} \theta_{2}, \beta>1 / 3 \sin ^{2} \theta_{2}\right\}$ and a Hamiltonian-Hopf bifurcation along the curve $\Gamma_{\theta_{2}}$. These situations are typically associated with bifurcations of small-amplitude homoclinic solutions from the zero equilibrium and can be investigated using Hamiltonian normal-form theory. The relevant investigations have been carried out in detail by Kirchgässner [20], Iooss \& Kirchgässner [15] and Buffoni \& Groves [3] in the context of two-dimensional travelling waves; the corresponding results for oblique line waves are obtained by a simple scaling of the solutions obtained in the above references and are stated below.

The Hamiltonian $0^{2}$ resonance along $\left\{(\beta, \alpha): \alpha=\sin ^{2} \theta_{2}, \beta>1 / 3 \sin ^{2} \theta_{2}\right\}$

We choose physical parameters $\left(\beta_{0}, \alpha_{0}\right)$ with $\beta_{0}>1 / 3 \sin ^{2} \theta_{2}, \alpha_{0}=\sin ^{2} \theta_{2}$ and introduce a bifurcation parameter by writing $\alpha=\alpha_{0}+\mu$.

Theorem 7 (Kirchgässner) For each sufficiently small, positive value of $\mu$ the one-degree-offreedom reduced Hamiltonian system has a unique, symmetric homoclinic solution. This homoclinic solution corresponds to an oblique line solitary wave of depression which is described by the formula

$$
\eta(x)=-\csc ^{2} \theta_{2}\left(\beta \csc ^{2} \theta_{2}-1 / 3\right)^{1 / 2} \mu \operatorname{sech}^{2}\left(\frac{3}{4\left(3 \beta-\sin ^{2} \theta_{2}\right)}\right)^{1 / 2} \mu^{1 / 2} x+\mathcal{O}\left(\mu^{3 / 2}\right) .
$$

and sketched in Figure 12. 


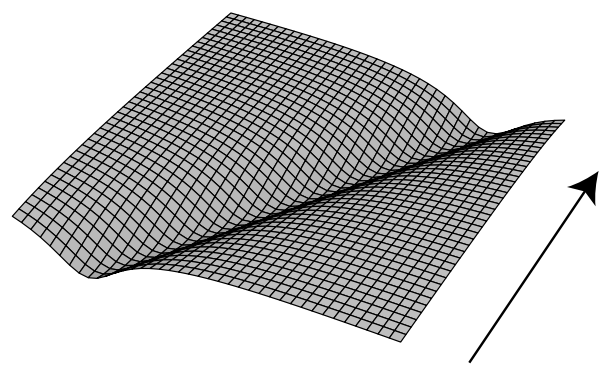

Figure 12: This wave has the profile of a solitary wave of depression in one distinguished spatial direction and is constant in another; it moves without change of shape in the direction indicated by the arrow.

\section{The Hamiltonian-Hopf bifurcation at points of $\Gamma_{\theta_{2}}$}

We choose physical parameters $\left(\beta_{0}, \alpha_{0}\right) \in \Gamma_{\theta_{2}}$ and introduce a bifurcation parameter by writing $\alpha=\alpha_{0}+\mu$.

\section{Theorem 8}

(i) (Iooss \& Kirchgässner) For each sufficiently small, positive value of $\mu$ the two-degreeof-freedom reduced Hamiltonian system has two distinct symmetric homoclinic solutions.

(ii) (Buffoni \& Groves) For each sufficiently small, positive value of $\mu$ the two-degreeof-freedom reduced Hamiltonian system has an infinite number of geometrically distinct homoclinic solutions which generically resemble multiple copies of one of the Iooss \& Kirchgässner homoclinics.

The homoclinic solutions identified above correspond to travelling water waves which are independent of $z$ and have an envelope solitary-wave profile in the $x$ direction whose amplitude is of order $\sqrt{\mu}$; they are sketched in Figure 13.
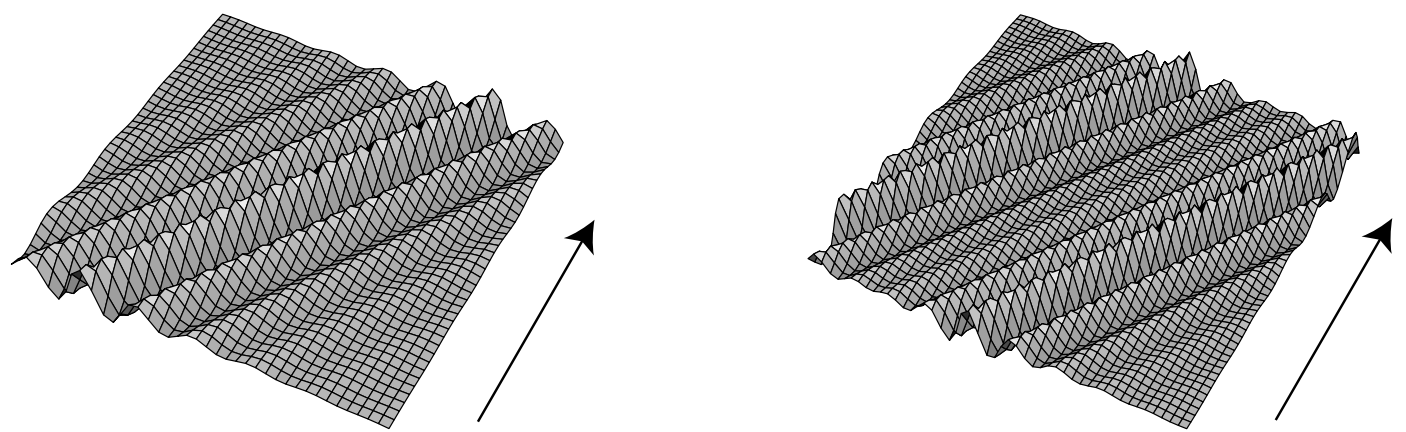

Figure 13: These waves have the profile of (a) one-pulse and (b) two-pulse envelope solitary waves in one distinguished spatial direction and are constant in another; they move without change of shape in the direction indicated by the arrow. 
Let us now turn to the general case in which eigenvalues associated with higher Fourier modes are also involved. A procedure for identifying all bifurcation scenarios obtained by fixing $\alpha, \beta, \theta_{1}, \theta_{2}$ and decreasing $\nu$ through a $\nu_{\mathrm{c}}$ is given in Section 3.3 above, where in particular a full catalogue of bifurcation scenarios involving only mode 0 , mode 1 and mode -1 eigenvalues is presented. In the material below we examine small-amplitude bifurcation phenomena which arise in two of these bifurcation scenarios, namely the Hamiltonian-Hopf bifurcation and the Hamiltonian $(\mathrm{i} \omega)^{2} \mathrm{i} \omega_{1}$ resonance.

\section{The Hamiltonian-Hopf bifurcation in cases $\mathrm{II}_{\mathrm{a}}$ and $\mathrm{III}_{\mathrm{a}}$}

A Hamiltonian-Hopf bifurcation involving only mode 1 and mode -1 eigenvalues occurs in the basic bifurcation scenarios referred to as cases $\mathrm{II}_{\mathrm{a}}$ and $\mathrm{III}_{\mathrm{a}}$ in Section 3.3 above: as $\nu$ is decreased through a critical value $\nu_{\mathrm{c}}$ four complex eigenvalues become purely imaginary by colliding in pairs on the imaginary axis, and we introduce a bifurcation parameter by writing $\nu=\nu_{\mathrm{c}}+\mu$. Observe that there are two degenerate versions of the Hamiltonian-Hopf bifurcation (both of which are included in the theory below), in which the eigenvalues are zero at criticality (see Figure 7); in one of these cases the eigenvalues are complex for $\mu>0$ while in the other $\left(\theta_{1}= \pm \pi / 2, \theta_{2}=0\right)$ they are real.

At criticality the central subspace of the linear operator $L$ consists of the geometrically simple eigenvalues 0 and $\pm \mathrm{i} s$, all of which have Jordan chains of length two. We choose a symplectic basis $\left\{e_{1}, f_{1}, e_{s}^{1} \mathrm{e}^{\mathrm{i} z}, \bar{e}_{s}^{1} \mathrm{e}^{-\mathrm{i} z}, f_{s}^{1} \mathrm{e}^{\mathrm{i} z}, f_{s}^{1} \mathrm{e}^{-\mathrm{i} z}\right\}$ for the six-dimensional centre manifold according to the procedure described in Section 4.1 above, so that $L e_{1}=0, L f_{1}=e_{1}, L e_{s}^{1} \mathrm{e}^{\mathrm{i} z}=\mathrm{i} e_{s}^{1} \mathrm{e}^{\mathrm{i} z}$, $(L-\mathrm{i} s I) f_{s}^{1} \mathrm{e}^{\mathrm{i} z}=e_{s}^{1} \mathrm{e}^{\mathrm{i} z}$ with $\Omega\left(e_{1}, f_{1}\right)=1, \Omega\left(e_{s}^{1} \mathrm{e}^{\mathrm{i} z}, \bar{f}_{s}^{1} \mathrm{e}^{-\mathrm{i} z}\right)=1, \Omega\left(f_{s}^{1} \mathrm{e}^{\mathrm{i} z}, \bar{e}_{s}^{1} \mathrm{e}^{-\mathrm{i} z}\right)=-1$, and we write

$$
u_{1}=q e_{1}+p f_{1}+A e_{s}^{1} \mathrm{e}^{\mathrm{i} z}+B f_{s}^{1} \mathrm{e}^{\mathrm{i} z}+\bar{A} \bar{e}_{s}^{1} \mathrm{e}^{-\mathrm{i} z}+\bar{B} \bar{f}_{s}^{1} \mathrm{e}^{-\mathrm{i} z}
$$

in particular

$$
e_{1}=\frac{1}{\sqrt{1-\sin ^{2} \theta_{2} / \alpha}}\left(\begin{array}{l}
0 \\
0 \\
1 \\
0
\end{array}\right), \quad f_{1}=\frac{1}{\sqrt{1-\sin ^{2} \theta_{2} / \alpha}}\left(\begin{array}{c}
\sin \theta_{2} / \alpha \\
0 \\
0 \\
1-\sin ^{2} \theta_{2} / \alpha
\end{array}\right)
$$

and

$$
e_{s}^{1}=\frac{\mathrm{e}^{\mathrm{i} z}}{\sqrt{\gamma_{1}}}\left(\begin{array}{c}
-\frac{\mathrm{i} \tilde{\Omega} \sinh \tilde{\Omega}}{\nu \sin \theta_{1}+s \sin \theta_{2}} \\
\cosh \tilde{\Omega} \sin \theta_{2}-\frac{1}{\tilde{\Omega}} \sinh \tilde{\Omega} \sin \theta_{2}-\frac{\beta \tilde{\Omega}\left(s+\nu \cos \left(\theta_{1}-\theta_{2}\right)\right) \sinh \tilde{\Omega}}{\nu \sin \theta_{1}+s \sin \theta_{2}} \\
\cosh \tilde{\Omega} y \\
\mathrm{i}\left(s+\nu \cos \left(\theta_{1}-\theta_{2}\right)\right) \cosh \tilde{\Omega} y-\frac{\mathrm{i} \tilde{\Omega} \sin \theta_{2} \sinh \tilde{\Omega}}{\nu \sin \theta_{1}+s \sin \theta_{2}}
\end{array}\right),
$$

where the formula for $\gamma_{1}$ is stated in Appendix A. The dimension of the centre manifold is reduced by two by eliminating the cyclic coordinate $q$, and the flow of the resulting fourdimensional Hamiltonian system can be analysed using the theory developed by Iooss \& Pérouème [17] and Buffoni \& Groves [3]. 
The Birkhoff normal-form theory states that for each $n_{0} \geq 2$ there is a near-identity, analytic, symplectic change of coordinates with the property that

$$
\begin{aligned}
\tilde{H}^{\mu}(A, B) & =\mathrm{i} q(A \bar{B}-\bar{A} B)+|B|^{2} \\
& +H_{\mathrm{NF}}\left(|A|^{2}, \mathrm{i}(A \bar{B}-\bar{A} B), \mu\right)+\mathcal{O}\left(|(A, B)|^{2}|(\mu, A, B)|^{n_{0}}\right)
\end{aligned}
$$

in the new coordinates; the function $H_{\mathrm{NF}}$ is a real polynomial of order $n_{0}+1$ which satisfies

$$
H_{\mathrm{NF}}\left(|A|^{2}, \mathrm{i}(A \bar{B}-\bar{A} B), \mu\right)=\mathcal{O}\left(|(A, B)|^{2}|(\mu, A, B)|\right) ;
$$

in these coordinates Hamilton's equations for the reduced system are given by

$$
\begin{aligned}
A_{x}= & \mathrm{i} q A+B+\mathrm{i} A \partial_{2} H_{\mathrm{NF}}\left(|A|^{2}, \mathrm{i}(A \bar{B}-\bar{A} B), \mu\right)+\mathcal{O}\left(|(A, B) \|(\mu, A, B)|^{n_{0}}\right), \\
B_{x}= & \mathrm{i} q B+\mathrm{i} B \partial_{2} H_{\mathrm{NF}}\left(|A|^{2}, \mathrm{i}(A \bar{B}-\bar{A} B), \mu\right) \\
& \quad-A \partial_{1} H_{\mathrm{NF}}\left(|A|^{2}, \mathrm{i}(A \bar{B}-\bar{A} B), \mu\right)+\mathcal{O}\left(|(A, B) \|(\mu, A, B)|^{n_{0}}\right) .
\end{aligned}
$$

The theory by Iooss \& Pérouème and Buffoni \& Groves demands that the coefficients $c_{1}$ and $c_{3}$ in the expansion

$$
\begin{aligned}
& H_{\mathrm{NF}}=\mu c_{1}|A|^{2}+\mu \mathrm{i} c_{2}(A \bar{B}-\bar{A} B)+c_{3}|A|^{4} \\
& \quad+\mathrm{i} c_{4}|A|^{2}(A \bar{B}-\bar{A} B)-c_{5}(A \bar{B}-\bar{A} B)^{2}+\mu^{2} c_{6}|A|^{2}+\mu^{2} \mathrm{i} c_{7}(A \bar{B}-\bar{A} B)+\ldots
\end{aligned}
$$

are respectively negative and positive; these coefficients are efficiently calculated using a procedure explained in detail by Groves \& Mielke [11, Appendix B] and are stated in Appendix A of the present paper.

\section{Theorem 9}

(i) (Iooss \& Pérouème) For each sufficiently small, positive value of $\mu$ the two-degree-offreedom Hamiltonian system (51), (52) has two distinct symmetric homoclinic solutions.

(ii) (Buffoni \& Groves) Suppose that $\left(\theta_{1}, \theta_{2}\right) \neq( \pm \pi / 2,0)$. For each sufficiently small, positive value of $\mu$ the two-degree-of-freedom Hamiltonian system (51), (52) has an infinite number of geometrically distinct homoclinic solutions which generically resemble multiple copies of one of the Iooss \& Pérouème homoclinics.

The homoclinic solutions identified above correspond to travelling water waves which are $2 \pi /\left(\nu_{c}+\mu\right)$-periodic in $z$ and have an envelope solitary-wave profile in the $x$ direction whose amplitude is of order $\sqrt{c_{1} \mu}$; they are sketched in Figure 14.

Finally note that in case $\mathrm{III}_{\mathrm{a}}$ the Hamiltonian-Hopf bifurcation at $\nu=\nu_{\mathrm{c}}$ may be followed by a second Hamiltonian-Hopf bifurcation as $\nu$ is decreased (see Figure 8). The above theory also applies in this case, but the signs of the bifurcation parameter $\mu$ and the coefficient $c_{1}$ are reversed. 

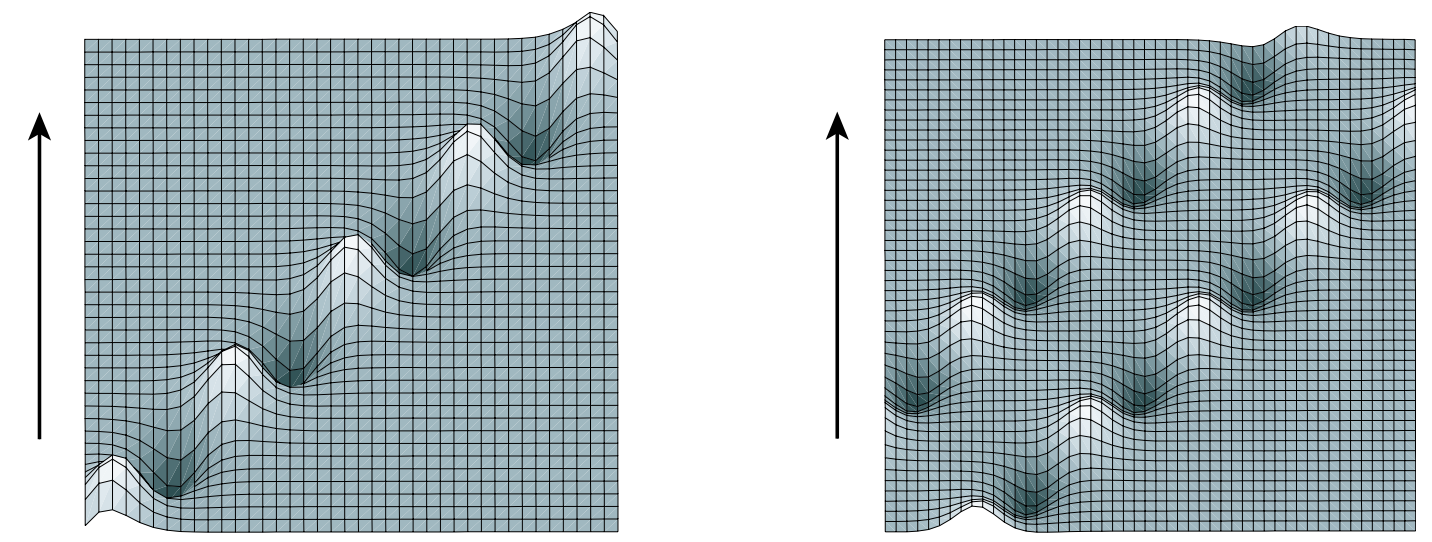

Figure 14: These waves have the profile of (a) one-pulse and (b) two-pulse envelope solitary wave in one distinguished spatial direction and are periodic in another; they move without change of shape in the direction indicated by the arrow.

\section{The Hamiltonian $\left(i \omega_{0}\right)^{2} i \omega_{1}$ resonance in case $I_{b}$}

A Hamiltonian $\left(\mathrm{i} \omega_{0}\right)^{2} \mathrm{i} \omega_{1}$ resonance occurs in the basic bifurcation scenario referred to as case $\mathrm{II}_{\mathrm{b}}$ in Section 3.3 above: as $\nu$ is decreased through a critical value $\nu_{\mathrm{c}}$ four complex mode \pm 1 eigenvalues become purely imaginary by colliding in pairs on the imaginary axis, and two nonzero purely imaginary mode 0 eigenvalues are present throughout the collision. (The degenerate case in which the mode \pm 1 eigenvalues are zero at criticality is included in the following theory.)

We introduce a bifurcation parameter by writing $\nu=\nu_{\mathrm{c}}+\mu$ and notice that at criticality the central subspace of the linear operator $L$ has a pair $\pm \mathrm{i} q$ of simple mode 0 eigenvalues and geometrically simple eigenvalues 0 (mode 0 ) and \pm is (mode \pm 1 ), all of which have Jordan chains of length two. We choose a symplectic basis $\left\{e_{1}, f_{1}, e_{q}^{0}, f_{q}^{0}, e_{s}^{1} \mathrm{e}^{\mathrm{i} z}, \bar{e}_{s}^{1} \mathrm{e}^{-\mathrm{i} z}, f_{s}^{1} \mathrm{e}^{\mathrm{i} z}, f_{s}^{1} \mathrm{e}^{-\mathrm{i} z}\right\}$ for the eight-dimensional centre manifold according to the procedure described in Section 4.1 above, so that $L e_{1}=0, L f_{1}=e_{1}, L e_{q}^{0}=\mathrm{i} q e_{q}^{0}, L \bar{e}_{q}^{0}=\mathrm{i} q \bar{e}_{q}^{0}, L e_{s}^{1} \mathrm{e}^{\mathrm{i} z}=\mathrm{i} e_{s}^{1} \mathrm{e}^{\mathrm{i} z},(L-\mathrm{i} s I) f_{s}^{1} \mathrm{e}^{\mathrm{i} z}=e_{s}^{1} \mathrm{e}^{\mathrm{i} z}$ with $\Omega\left(e_{1}, f_{1}\right)=1, \Omega\left(e_{q}^{0}, \bar{e}_{q}^{0}\right)=-\mathrm{i}, \Omega\left(e_{s}^{1} \mathrm{e}^{\mathrm{i} z}, \overline{f_{s}^{1}} \mathrm{e}^{-\mathrm{i} z}\right)=1, \Omega\left(f_{s}^{1} \mathrm{e}^{\mathrm{i} z}, \bar{e}_{s}^{1} \mathrm{e}^{-\mathrm{i} z}\right)=-1$, and we write

$$
u_{1}=q e_{1}+p f_{1}+C e_{q}^{0}+\bar{C} \bar{e}_{q}^{0}+A e_{s}^{1} \mathrm{e}^{\mathrm{i} z}+B f_{s}^{1} \mathrm{e}^{\mathrm{i} z}+\bar{A} \bar{e}_{s}^{1} \mathrm{e}^{-\mathrm{i} z}+\bar{B} \bar{f}_{s}^{1} \mathrm{e}^{-\mathrm{i} z}
$$

in particular we have that

$$
e_{1}=\frac{1}{\sqrt{\sin ^{2} \theta_{2} / \alpha-1}}\left(\begin{array}{c}
\sin \theta_{2} / \alpha \\
0 \\
0 \\
1-\sin ^{2} \theta_{2} / \alpha
\end{array}\right), \quad f_{1}=\frac{1}{\sqrt{\sin ^{2} \theta_{2} / \alpha-1}}\left(\begin{array}{l}
0 \\
0 \\
1 \\
0
\end{array}\right)
$$

and $e_{s}^{1}$ and $e_{q}^{0}$ are given by respectively (49) and (45) with normalisation factor

$$
\left.\left(-4 \alpha \csc ^{2} \theta_{2} \sinh ^{2} q+q(2 q+\sinh 2 q)\right) /(2 q)\right)^{-1 / 2} .
$$

The dimension of the centre manifold is reduced by two by eliminating the cyclic variable $p$, and the flow of the resulting six-dimensional Hamiltonian system can be analysed using the theory developed by Groves \& Mielke [11, §4] and Lombardi [22, Chapter 9]. 
Under the assumption that $q / s \notin \mathbb{Q}$, the Birkhoff normal-form theory states that for each $n_{0} \geq 2$ there is a near-identity, analytic, symplectic change of coordinates with the property that

$$
\begin{aligned}
& \tilde{H}^{\mu}(A, B)=\mathrm{i} s(A \bar{B}-\bar{A} B)+|B|^{2}+q|C|^{2} \\
& \quad+H_{\mathrm{NF}}\left(|A|^{2}, \mathrm{i}(A \bar{B}-\bar{A} B),|C|^{2}, \mu\right)+\mathcal{O}\left(|(A, B, C)|^{2}|(\mu, A, B, C)|^{n_{0}}\right)
\end{aligned}
$$

in the new coordinates; the function $H_{\mathrm{NF}}$ is a real polynomial of order $n_{0}+1$ which satisfies

$$
H_{\mathrm{NF}}\left(|A|^{2}, \mathrm{i}(A \bar{B}-\bar{A} B),|C|^{2}, \mu\right)=\mathcal{O}\left(|(A, B, C)|^{2}|(\mu, A, B, C)|\right) ;
$$

in these coordinates Hamilton's equations for the reduced system are given by

$$
\begin{aligned}
A_{x}= & \mathrm{i} s A+B+\mathrm{i} A \partial_{2} H_{\mathrm{NF}}\left(|A|^{2}, \mathrm{i}(A \bar{B}-\bar{A} B),|C|^{2}, \mu\right)+\mathcal{O}\left(|(A, B, C)||(\mu, A, B, C)|^{n_{0}}\right), \\
B_{x}= & \mathrm{i} s B+\mathrm{i} B \partial_{2} H_{\mathrm{NF}}\left(|A|^{2}, \mathrm{i}(A \bar{B}-\bar{A} B),|C|^{2}, \mu\right) \\
& \quad-A \partial_{1} H_{\mathrm{NF}}\left(|A|^{2}, \mathrm{i}(A \bar{B}-\bar{A} B),|C|^{2} ; \mu\right)+\mathcal{O}\left(|(A, B, C)||(\mu, A, B, C)|^{n_{0}}\right), \\
C_{x}= & \mathrm{i} q C+\mathrm{i} C \partial_{3} H_{\mathrm{NF}}\left(|A|^{2}, \mathrm{i}(A \bar{B}-\bar{A} B),|C|^{2}, \mu\right)+\mathcal{O}\left(|(A, B, C)||(\mu, A, B, C)|^{n_{0}}\right) .
\end{aligned}
$$

The theory by Groves \& Mielke and Lombardi demands that the coefficients $c_{1}, c_{4}$ and $c_{6}$ in the expansion

$$
\begin{gathered}
H_{\mathrm{NF}}=c_{1} \mu|A|^{2}+\mathrm{i} c_{2} \mu(A \bar{B}-\bar{A} B)+c_{3} \mu|C|^{2}+c_{4}|A|^{4}-c_{5}(A \bar{B}-\bar{A} B)^{2}+c_{6}|C|^{4} \\
+\mathrm{i} c_{7}|A|^{2}(A \bar{B}-\bar{A} B)+c_{8}|A|^{2}|C|^{2}+\mathrm{i} c_{9}(A \bar{B}-\bar{A} B)|C|^{2}+\ldots
\end{gathered}
$$

satisfy $c_{1}<0, c_{4}>0$ and $c_{6} \neq 0$; expressions for these coefficients are given in Appendix A.

Theorem 10 (Lombardi) Consider the Hamiltonian $\left(\mathrm{i} \omega_{0}\right)^{2} \mathrm{i} \omega_{1}$ resonance in case $I I_{\mathrm{b}}$. Let $m$ be any natural number. There exist positive constants $\varepsilon_{1}, \varepsilon_{2}, \varepsilon_{3}$ such that the following statements hold for each sufficiently small, positive value of $\mu$.

(i) The reduced sixth-order Hamiltonian system has an invariant subspace $\{A=B=0\}$ containing a Lyapunov family of periodic orbits $X_{\varepsilon, \mu}$ of arbitrarily small amplitude $\varepsilon \in$ $\left[0, \varepsilon_{1} \mu^{7 / 4}\right]$.

(ii) The reduced sixth-order Hamiltonian system has a pair of reversible homoclinic connections to $X_{\varepsilon, \mu}$, for every $\varepsilon \in\left[\varepsilon_{2} \mu^{m}, \varepsilon_{3} \mu^{7 / 4}\right]$.

The homoclinic solutions identified above correspond to travelling water waves which are $2 \pi /\left(\nu_{\mathrm{c}}+\mu\right)$-periodic in $z$ and have the profile of a generalised solitary wave in the $x$ direction with central 'localised' part of amplitude $\sqrt{\left|c_{1}\right| \mu}$; as $x \rightarrow \pm \infty$ the free-surface displacement converges to a z-independent periodic wave of ampltiude $\varepsilon$ (see Figure 15).

We conclude with a brief commentary on the cases $\theta_{1}=0, \theta_{2}= \pm \pi / 2$ and $\theta_{1}= \pm \pi / 2, \theta_{2}=$ 0 . In these special situations Hamilton's equations possess an additional reflection symmetry since the equations (12)-(15) are invariant under both (9) and (10). This symmetry is inherited by the reduced equations on the centre manifold and may be exploited to halve the dimension of the reduced Hamiltonian system. At the linear level this symmetry manifests itself in the fact that all eigenvalues of the corresponding linear operator are geomtrically double, half of them corresponding to wave motions which are respectively symmetric and antisymmetric with respect to the symmetry operator. These special cases, and the symmetry reductions involved in them, have been discussed in detail by Groves \& Mielke [11], Groves [10] and Haragus \& Kirchgässner [13]. 


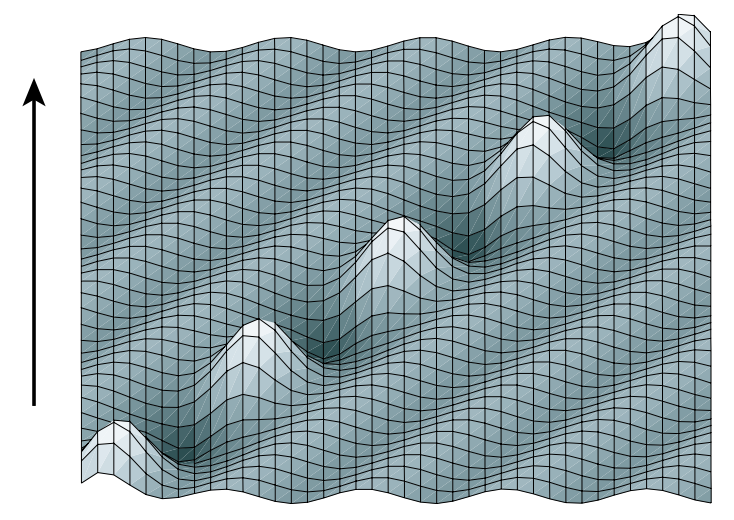

Figure 15: This wave has a generalised solitary-wave profile in one distinguished spatial direction and is periodic in another; it moves without change of shape in the direction indicated by the arrow.

\section{Appendix A Normal-form coefficients}

\section{The $-1: 2$ resonance at points of $R_{2}$}

The coefficients in the formula (48) for the Hamiltonian in Birkhoff normal form are given by

$$
\begin{aligned}
& c_{1}=-9 q^{2} \cosh q \csc ^{2} \theta_{2} \sinh q \\
& \times[4\left(4021+9072 q^{2}\right) \cosh q+2 q\left(3281+9216 q^{2}\right) \cosh 3 q \\
&+16(56 \cosh q+15 \cosh 3 q+\cosh 5 q)^{2} \sinh ^{3} q \\
&+2 q(-5041 \cosh 7 q-1337 \cosh 9 q-144 \cosh 11 q-7 \cosh 13 q \\
&+6\left(\left(-799+1332 q^{2}\right) \cosh 5 q\right. \\
&+4 q(3 q(69 \cosh 7 q+15 \cosh 9 q+\cosh 11 q)-13 \sinh q \\
&+569 \sinh 3 q+623 \sinh 5 q+175 \sinh 7 q+4 \sinh 9 q-2 \sinh 11 q)))] \\
& \times[8(2\left.+\cosh 2 q)(-12 q \cosh q+9 \sinh q+\sinh 3 q)^{3}(12 q-8 \sinh 2 q+\sinh 4 q)\right]^{-1}, \\
& c_{2}=\quad \\
& 9 q^{2} \operatorname{coth}{ }^{2} q \csc { }^{2} \theta_{2} \operatorname{csch} q \\
& \times\left[-158541 q+559008 q^{3}+11474 \sinh 2 q-6790 \sinh 4 q-1206 \sinh 6 q\right. \\
& \quad+1538 \sinh 8 q+98 \sinh 10 q-126 \sinh 12 q-22 \sinh 14 q-\sinh 16 q \\
& \quad+q\left(\left(89833+841464 q^{2}\right) \cosh 2 q+11183 \cosh 6 q+25532 \cosh 8 q\right. \\
& \quad+13333 \cosh 10 q+3016 \cosh 12 q+339 \cosh 14 q+17 \cosh 16 q \\
& \quad-12\left(-14\left(91+2802 q^{2}\right) \cosh 4 q+q(-6 q(3635 \cosh 6 q+1228 \cosh 8 q\right. \\
&\quad+229 \cosh 10 q+22 \cosh 12 q+\cosh 14 q)+40726 \sinh 2 q+18480 \sinh 4 q \\
&+17614 \sinh 6 q+9902 \sinh 8 q+2774 \sinh 10 q \\
&+400 \sinh 12 q+30 \sinh 14 q+\sinh 16 q)))] \\
& \times\left[16(2+\cosh 2 q)(-12 q \cosh q+9 \sinh q+\sinh 3 q)^{3}(12 q-8 \sinh 2 q+\sinh 4 q)\right]^{-1},
\end{aligned}
$$




$$
\begin{aligned}
c_{3}= & 9 q^{3} \operatorname{coth} q \csc ^{2} \theta_{2} \operatorname{csch}^{2} q \\
& \times(146+140 \cosh 2 q+23 \cosh 4 q+78 \cosh 6 q+38 \cosh 8 q+6 \cosh 10 q+\cosh 12 q) \\
& \times\left[2(2+\cosh 2 q)(12 q-8 \sinh 2 q+\sinh 4 q)^{2}\right]^{-1} .
\end{aligned}
$$

\section{The $-1: 3$ resonance at points of $R_{3}$}

The coefficients in the formula (47) for the Hamiltonian in Birkhoff normal form are given by

$$
\begin{aligned}
c_{1}= & -q^{3}(1+2 \cosh 2 q) \csc ^{2} \theta_{2} \operatorname{csch}^{3} q \operatorname{sech}^{3} q \\
& \times(109634+192616 \cosh 2 q+121897 \cosh 4 q \\
& \quad+55780 \cosh 6 q+15022 \cosh 8 q+2484 \cosh 10 q+231 \cosh 12 q) \\
& \times\left[768(-4 q-8 q \cosh 2 q+4 \sinh 2 q+\sinh 4 q)^{2}\right]^{-1}, \\
c_{2}= & 3 q^{3} \csc ^{2} \theta_{2} \operatorname{csch}^{3} q \operatorname{sech}^{3} q \\
& (369855+667539 \cosh 2 q+493080 \cosh 4 q+282937 \cosh 6 q+125052 \cosh 8 q \\
& \quad+41595 \cosh 10 q+9320 \cosh 12 q+1209 \cosh 14 q+69 \cosh 16 q) \\
& \times\left[12 q 6(4+3 \cosh 2 q)(4 q-5 \cosh q \sinh 2 q-3 \sinh 4 q+\sinh 6 q]^{-1},\right. \\
c_{3}= & \left.27 q^{3}\left(1+2 \cosh 3 q \sinh ^{2} q\right)\right]^{-1} \\
& \times(7381+1671 \cosh 2 q+2373 \cosh 4 q+2819 \cosh 6 q+114 \cosh 8 q+2409 \cosh 10 q \\
& \left.\quad+1291 \cosh 12 q+240 \cosh ^{3} 14 q+105 \cosh 16 q+29 \cosh 18 q\right) \\
& \times\left[256(11+10 \cosh 2 q)(12 q-3 \sinh 2 q-3 \sinh 4 q+\sinh 6 q)^{2}\right]^{-1} .
\end{aligned}
$$

In particular, we find that the quantities $m=-c_{2}-3 c_{1}, n=-c_{3}-3 c_{2}$ have the properties that the cubic polynomial $x^{3}+m x^{2}+9 x+n$ always has one real root and that $n$ is always positive.

\section{The $-1: 4$ resonance at points of $R_{4}$}

The coefficients in the formula (46) for the Hamiltonian in Birkhoff normal form are given by

$$
\begin{aligned}
c_{1}= & -45 q^{3} \cosh 2 q \operatorname{coth} q \csc ^{2} \theta_{2} \operatorname{csch}^{2} q \\
& \times(75744+143863 \cosh 2 q+106288 \cosh 4 q+63211 \cosh 6 q+32096 \cosh 8 q \\
& \quad+8981 \cosh 10 q+1616 \cosh 12 q+201 \cosh 14 q) \\
& \times\left[16(4+8 \cosh 2 q+3 \cosh 4 q)(-60 q(\cosh q+\cosh 3 q)+25 \sinh 3 q+9 \sinh 5 q)^{2}\right]^{-1}
\end{aligned}
$$




$$
\begin{aligned}
c_{2}= & 45 q^{3} \operatorname{coth} q \csc ^{2} \theta_{2} \operatorname{csch}^{2} q \\
& \times(3007448+5633998 \cosh 2 q+4806712 \cosh 4 q+3605238 \cosh 6 q \\
& \quad+2321376 \cosh 8 q+1284901 \cosh 10 q+612120 \cosh 12 q \\
& \quad+240621 \cosh 14 q+71304 \cosh 16 q+14389 \cosh 18 q \\
& \quad+1776 \cosh 20 q+117 \cosh 22 q) \\
& \times[8(4+3 \cosh 2 q)(6+7 \cosh 2 q+2 \cosh 4 q)(4+8 \cosh 2 q+3 \cosh 4 q)]^{-1} \\
& \times\left[\left(30 q+16 \operatorname{coth} 4 q \sinh q^{2}-17 \sinh 2 q\right)\left(120 q-16 \operatorname{coth} q \sinh ^{2} 4 q+17 \sinh 8 q\right)\right]^{-1}, \\
c_{3}= & 90 q^{3} \csc \cos ^{2} \operatorname{csch}^{3} q \\
& \times(26704 \cosh q+27856 \cosh 3 q+10087 \cosh 5 q+8241 \cosh 7 q+7867 \cosh 9 q \\
& \quad+8325 \cosh 11 q+4920 \cosh 13 q+5936 \cosh 15 q+4840 \cosh 17 q \\
& \quad+2144 \cosh 19 q+635 \cosh 21 q+285 \cosh 23 q+127 \cosh 25 q+33 \cosh 27 q) \\
& \times[(4+8 \cosh 2 q+3 \cosh 4 q)(15+20 \cosh 2 q+7 \cosh 4 q)]^{-1} \\
& \times[-120 q+16 \sinh 2 q+16 \sinh 4 q+16 \sinh 6 q-9 \sinh 8 q]^{-2} .
\end{aligned}
$$

In particular, we find that the quantities $m=-c_{2}-4 c_{1}, n=-c_{3}-4 c_{2}$ are always positive.

\section{The Hamiltonian-Hopf bifurcation in cases $\mathrm{II}_{\mathrm{a}}$ and $\mathrm{III}_{\mathrm{a}}$}

The coefficients in the formula (50) for the Hamiltonian in Birkhoff normal form are given by

$$
\begin{gathered}
c_{1}=-4 b \tilde{\gamma}^{4} a \cosh \tilde{\gamma} \sin \theta_{2} \\
{\left[\operatorname { c o s h } \tilde { \gamma } \left(-b^{2} a\left(3 a^{2}+\tilde{\gamma}\left(a^{2}-\tilde{\gamma}^{2}\right) \operatorname{coth} \tilde{\gamma}\right)\right.\right.} \\
\left.\quad+2 b \tilde{\gamma}^{2}\left(2 a^{2}+\tilde{\gamma}^{2}+2 a^{2} \tilde{\gamma} \operatorname{coth} \tilde{\gamma}\right) \sin \theta_{2}-2 a \tilde{\gamma}^{4} \sin ^{2} \theta_{2}\right) \\
+b a \tilde{\gamma}\left(-b\left(2 a^{2}+\tilde{\gamma}^{2}+2 a^{2} \tilde{\gamma} \operatorname{coth} \tilde{\gamma}\right) \operatorname{csch} \tilde{\gamma}\right. \\
\left.\left.\quad+\left(b\left(a^{2}-\tilde{\gamma}^{2}\right)-4 a \tilde{\gamma}^{2} \sin \theta_{2}\right) \sinh \tilde{\gamma}\right)\right]^{-1},
\end{gathered}
$$




$$
\begin{aligned}
& \gamma_{1}^{2} c_{3} \\
& =\frac{\pi}{12 \alpha b^{2} \tilde{\gamma}} \\
& \left(-4 \tilde{\gamma} \sinh ^{2} \tilde{\gamma}\left(\alpha a \tilde{\gamma}^{2}\left(6 a-2 a \tilde{\gamma}^{2}+4 \nu \tilde{\gamma}^{2} \cos \left(\theta_{1}-\theta_{2}\right)+3 a \cosh 2 \tilde{\gamma}\right)\right.\right. \\
& \left.+6 b \nu \tilde{\gamma}^{2} \cos \left(\theta_{1}-\theta_{2}\right) \sin \theta_{2}\right) \\
& -12\left(\alpha a^{2} \tilde{\gamma}^{2}\left(1+2 \tilde{\gamma}^{2}\right)-4 \alpha a \nu \tilde{\gamma}^{4} \cos \left(\theta_{1}-\theta_{2}\right)\right) \cosh \tilde{\gamma} \sinh ^{3} \tilde{\gamma} \\
& \left.+12 b \tilde{\gamma}^{4}\left(b \tilde{\gamma}+\nu \cos \left(\theta_{1}-\theta_{2}\right) \sin \theta_{2} \sinh 2 \tilde{\gamma}\right)\right) \\
& +\frac{\pi}{32 \beta b^{4} \tilde{\gamma}^{2}} \\
& \left(4 b^{4} a^{2} \tilde{\gamma}^{2} \cosh ^{2} 2 \tilde{\gamma}^{2}\right. \\
& +8 \beta b^{2} \tilde{\gamma}^{4} \cosh 2 \tilde{\gamma} \sinh ^{2} \tilde{\gamma}\left(4 a^{2}+3 \nu^{2}-8 a \nu \cos \left(\theta_{1}-\theta_{2}\right)-3 \nu^{2} \cos 2\left(\theta_{1}-\theta_{2}\right)\right) \\
& +32 \beta b^{2} \tilde{\gamma}^{4} \sinh ^{2} \tilde{\gamma}\left(3 a^{2}+\tilde{\gamma}^{2}+\nu^{2} \tilde{\gamma}^{2} \sin ^{2}\left(\theta_{1}-\theta_{2}\right)\right) \\
& +8 \beta b^{2} \tilde{\gamma}^{3} \cosh \tilde{\gamma} \sinh ^{3} \tilde{\gamma}\left(8 a^{2}-3 \nu^{2}-2\left(2+3 \nu^{2} \tilde{\gamma}^{2}\right.\right. \\
& \left.+\nu\left(8 a \cos \left(\theta_{1}-\theta_{2}\right)+3 \nu\left(1+2 \tilde{\gamma}^{2}\right) \cos 2\left(\theta_{1}-\theta_{2}\right)\right)\right) \\
& \left.+48 \beta^{2} \tilde{\gamma}^{10} \sinh ^{4} \tilde{\gamma}+b^{4} a^{2}\left(\sinh ^{2} 2 \tilde{\gamma}-2 \tilde{\gamma} \sinh 4 \tilde{\gamma}\right)\right) \\
& +\frac{\pi}{48 \beta b^{3} \tilde{\gamma}^{2}} \\
& \left(-6 b^{3} a^{2} \tilde{\gamma}^{2} \cosh ^{2} 2 \tilde{\gamma}\right. \\
& -2\left(3 b^{3} a^{2} \cosh ^{2} \tilde{\gamma}\right. \\
& +2 \beta \tilde{\gamma}^{4}\left(2 b \left(6 \tilde{\gamma}^{2}+a^{2}\left(6+4 \tilde{\gamma}^{2}\right)+9 \nu^{2} \cosh 2 \tilde{\gamma} \sin ^{2}\left(\theta_{1}-\theta_{2}\right)\right.\right. \\
& \left.+2 \nu \tilde{\gamma}^{2}\left(-4 a \cos \left(\theta_{1}-\theta_{2}\right)+3 \nu \sin ^{2}\left(\theta_{1}-\theta_{2}\right)\right)\right) \\
& -3 a\left(a^{2}-\tilde{\gamma}^{2}+2 a\left(a-2 \nu \cos \left(\theta_{1}-\theta_{2}\right)\right) \cosh 2 \tilde{\gamma}\right. \\
& \left.\left.\left.+\nu^{2}(1+2 \cosh 2 \tilde{\gamma}) \sin ^{2}\left(\theta_{1}-\theta_{2}\right)\right) \sin \theta_{2}\right)\right) \sinh ^{2} \tilde{\gamma} \\
& +12 \beta \tilde{\gamma}^{3} \cosh \tilde{\gamma}\left(2 b\left(-a^{2}+\tilde{\gamma}^{2}\right)+a\left(-a^{2}+\tilde{\gamma}^{2}+4 a \nu \cos \left(\theta_{1}-\theta_{2}\right)\right) \sin \theta_{2}\right. \\
& \left.+\nu^{2} \sin ^{2}\left(\theta_{1}-\theta_{2}\right)\left(4 b\left(2+\tilde{\gamma}^{2}\right)-a \sin \theta_{2}\right)\right) \sinh ^{3} \tilde{\gamma} \\
& +3 \beta b \tilde{\gamma}^{4}\left(2 \tilde{\gamma}^{2}-8 a \nu \cos \left(\theta_{1}-\theta_{2}\right)\right) \sinh ^{2} 2 \tilde{\gamma} \\
& +3 b \tilde{\gamma}\left(a^{2}\left(b^{2}-4 \beta \tilde{\gamma}^{4}\right)\right. \\
& \left.\left.-4 \beta \nu \tilde{\gamma}^{4}\left(-2 a \cos \left(\theta_{1}-\theta_{2}\right)+\nu \sin ^{2}\left(\theta_{1}-\theta_{2}\right)\right)\right) \sinh 4 \tilde{\gamma}\right) \\
& +\pi \sinh \tilde{\gamma}\left[48 b^{3} \tilde{\gamma}\left(-4 b^{2} \cosh 2 \tilde{\gamma}+2 \tilde{\gamma}\left(\alpha+4 \beta \tilde{\gamma}^{2}\right) \sinh 2 \tilde{\gamma}\right)\right]^{-1} \\
& {\left[-12 \tilde{\gamma}\left(-6 a^{2}-3 \nu^{2}+\tilde{\gamma}^{2}+10 a \nu \cos \left(\theta_{1}-\theta_{2}\right)+3 \nu^{2} \cos 2\left(\theta_{1}-\theta_{2}\right)\right) \cosh \tilde{\gamma}\right.} \\
& +6 \tilde{\gamma}\left(2 \tilde{\gamma}^{2}-4 a \nu \cos \left(\theta_{1}-\theta_{2}\right)\right) \cosh 3 \tilde{\gamma} \\
& +\sinh \tilde{\gamma}\left(-11\left(2 a^{2}+\nu^{2}-2 \tilde{\gamma}^{2}\right)+\nu\left(120 a \cos \left(\theta_{1}-\theta_{2}\right)+11 \nu \cos 2\left(\theta_{1}-\theta_{2}\right)\right)\right. \\
& \left.\left.+24 a \nu \cos \left(\theta_{1}-\theta_{2}\right) \cosh 2 \tilde{\gamma}\right)\right] \\
& {\left[4 b^{3} \tilde{\gamma}^{3} \cosh 2 \tilde{\gamma}\right.} \\
& -4\left(4 b^{3} \tilde{\gamma}^{3}+\left(\alpha a \tilde{\gamma}^{3}+4 \beta a \tilde{\gamma}^{5}-2 b^{2} \nu \tilde{\gamma} \cos \left(\theta_{1}-\theta_{2}\right)\right) \sin \theta_{2}\right) \sinh ^{2} \tilde{\gamma} \\
& +b \sinh 2 \tilde{\gamma}\left(-b^{2} a^{2}+b^{2} \tilde{\gamma}^{2}+2 \alpha a^{2} \tilde{\gamma}^{2}+2 \alpha \tilde{\gamma}^{4}+8 \beta a^{2} \tilde{\gamma}^{4}+8 \beta \tilde{\gamma}^{6}\right. \\
& \left.\left.-\nu^{2}\left(b^{2}-2 \tilde{\gamma}^{2}\left(\alpha+4 \beta \tilde{\gamma}^{2}\right)\right) \sin ^{2}\left(\theta_{1}-\theta_{2}\right)-4 b \nu \tilde{\gamma}^{2} \cos \left(\theta_{1}-\theta_{2}\right) \sin \theta_{2}\right)\right],
\end{aligned}
$$


where $a=s+\nu \cos \left(\theta_{1}-\theta_{2}\right), b=s \sin \theta_{2}+\nu \sin \theta_{1}$ and

$$
\begin{aligned}
\gamma_{1}= & \frac{\pi}{b^{3} \tilde{\gamma}^{2}}\left(b\left(b^{2} a^{2}+\beta \tilde{\gamma}^{2}\left(2 a^{2}+\tilde{\gamma}^{2}\right)\right)-2 a \tilde{\gamma}^{2}\left(b^{2}+\beta \tilde{\gamma}^{2}\right) \sin \theta_{2}\right) \cosh 2 \tilde{\gamma} \\
& +\frac{\pi}{2 b^{2} \tilde{\gamma}^{3}}\left(\left(1+4 \beta a^{2}\right) \tilde{\gamma}^{4}+b^{2}\left(\tilde{\gamma}^{2}-a^{2}\right)-\tilde{\gamma}^{4} \cos 2 \theta_{2}-2 b a \tilde{\gamma}^{2} \sin \theta_{2}\right) \sinh 2 \tilde{\gamma} \\
& +\frac{\pi}{b^{3}}\left(b\left(b^{2}-\beta\left(2 a^{2}+\tilde{\gamma}^{2}\right)\right)+2 \beta a \tilde{\gamma}^{2} \sin \theta_{2}\right) .
\end{aligned}
$$

In particular, we find that $c_{1}<0, c_{3}>0$.

\section{The Hamiltonian $\left(i \omega_{0}\right)^{2} i \omega_{1}$ resonance in case $I_{b}$}

The coefficients $c_{1}$ and $c_{4}$ in the formula (53) for the Hamiltonian in Birkhoff normal form are given by the formulae in the previous section for respectively $c_{1}$ and $c_{3}$ and

$$
\begin{aligned}
c_{6}= & q^{4}\left(8 q\left(3\left(8 q^{2}+\alpha^{2}\right)+8 q^{2}\left(-4 \cos 2 \theta_{2}+\cos 4 \theta_{2}\right)\right) \sin ^{2} \theta_{2}-216 q \alpha^{2} \cosh 4 q \sin ^{2} \theta_{2}\right. \\
& +4 q\left(24 q^{2}+51 \alpha^{2}+8 q^{2}\left(-4 \cos 2 \theta_{2}+\cos 4 \theta_{2}\right)\right) \cosh 2 q \sin ^{2} \theta_{2} \\
& +\alpha\left(-12 q \alpha \cosh 6 q \sin ^{2} \theta_{2}+8 q^{2}\left(-4 \cos \theta_{2}+\cos 4 \theta_{2}\right) \cosh ^{3} q(27 \sinh q+\sinh 3 q)\right. \\
& \left.\left.\quad+3\left(\left(57 q^{2}+60 \alpha^{2}\right) \sinh 2 q+6\left(5 q^{2}-8 \alpha^{2}\right) \sinh 4 q+\left(q^{2}+12 \alpha^{2}\right) \sinh 6 q\right)\right)\right) \\
\times & {\left[64 \alpha\left(2 q(2+\cosh 2 q) \sin ^{2} \theta_{2}-3 \alpha \sinh 2 q\right]^{-1}\right.} \\
\times & {\left[q^{2}+2 \alpha+q^{2} \cos 2 \theta_{2}-2 \alpha \cosh 2 q+q \sin ^{2} \theta_{2} \sinh 2 q\right]^{-2} . }
\end{aligned}
$$

In particular, we find that $c_{1}<0, c_{4}>0$ and $c_{6} \neq 0$.

\section{References}

[1] Akylas, T. 1994 Three-dimensional long water-wave phenomena. Ann. Rev. Fluid Mech. 26, 191-210.

[2] Ambrosetti, A. \& Prodi, G. 1995 A Primer of Nonlinear Analysis, paperback edn. Cambridge: C.U.P. (Cambridge studies in advanced mathematics 34)

[3] Buffoni, B. \& Groves, M. D. 1999 A multiplicity result for solitary gravity-capillary waves in deep water via critical-point theory. Arch. Rat. Mech. Anal. 146, 183-220.

[4] Buffoni, B., Groves, M. D. \& Toland, J. F. 1996 A plethora of solitary gravitycapillary water waves with nearly critical Bond and Froude numbers. Phil. Trans. Roy. Soc. Lond. A 354, 575-607.

[5] Craig, W. \& Nicholls, D. P. 2000 Traveling two and three dimensional capillary gravity water waves. SIAM J. Math. Anal. 32, 323-359.

[6] De Bouard, A. \& SAut, J.-C. 1997 Solitary waves of generalized KadomtsevPetviashvili equations. Ann. Inst. H. Poincaré Anal. Non Linéaire 14, 211-236.

[7] DiAs, F. \& Iooss, G. 2002 Water-waves as a spatial dynamical system. Preprint. (In Handbook of Mathematical Fluid Dynamics (eds. Friedlander, S. and Serre, D.), to appear) 
[8] DiAs, F. \& Kharif, C. 1999 Nonlinear gravity and capillary-gravity waves. Ann. Rev. Fluid Mech. 31, 301-346.

[9] Duistermant, J. J. 1984 Bifurcation of periodic solutions near equilibrium points of Hamiltonian systems. In Lecture Notes in Mathematics 1057 - Bifurcation Theory and Applications, Montecatini 1983 (ed. Salvadori, L.), pages 57-105. New York: SpringerVerlag.

[10] Groves, M. D. 2001 An existence theory for three-dimensional periodic travelling gravity-capillary water waves with bounded transverse profiles. Physica D 152-153, 395415.

[11] Groves, M. D. \& Mielke, A. 2001 A spatial dynamics approach to three-dimensional gravity-capillary steady water waves. Proc. Roy. Soc. Edin. A 131, 83-136.

[12] Hammack, J., Scheffner, N. \& Segur, H. 1989 Two-dimensional periodic waves in shallow water. J. Fluid Mech. 209, 567-589.

[13] Haragus-Courcelle, M. \& Kirchgässner, K. 2001 Three-dimensional steady capillary-gravity waves. In Ergodic Theory, Analysis, and Efficient Simulation of Dynamical Systems (ed. Fiedler, B.), pages 363-397. Berlin: Springer-Verlag.

[14] Haragus-Courcelle, M. \& Pego, R. L. 2000 Spatial wave dynamics of steady oblique wave interactions. Physica D 145, 207-232.

[15] IoOss, G. \& KIRCHGÄSSnER, K. 1990 Bifurcation d'ondes solitaires en présence d'une faible tension superficielle. C. R. Acad. Sci. Paris, Sér. 1 311, 265-268.

[16] IoOss, G. \& KIRCHGÄSSNER, K. 1992 Water waves for small surface tension: an approach via normal form. Proc. Roy. Soc. Edin. A 122, 267-299.

[17] Iooss, G. \& PÉrouÈme, M. C. 1993 Perturbed homoclinic solutions in reversible 1:1 resonance vector fields. J. Diff. Eqns. 102, 62-88.

[18] Jones, M. C. W. 1989 Small amplitude gravity-capillary waves in a channel of finite depth. Glasgow Math. J. 31, 141-160.

[19] KirchgäsSnER, K. 1982 Wave solutions of reversible systems and applications. J. Diff. Eqns. 45, 113-127.

[20] KIRCHGÄSSNER, K. 1988 Nonlinear resonant surface waves and homoclinic bifurcation. Adv. Appl. Math. 26, 135-181.

[21] LOMBARDI, E. 1997 Orbits homoclinic to exponentially small periodic orbits for a class of reversible systems. Application to water waves. Arch. Rat. Mech. Anal. 137, 227-304.

[22] LombARDI, E. 2000 Oscillatory Integrals and Phenomena Beyond all Algebraic Orders. Berlin: Springer-Verlag. 
[23] LuKE, J. C. 1967 A variational principle for a fluid with a free surface. J. Fluid Mech. 27, 395-397.

[24] Mielke, A. 1988 Reduction of quasilinear elliptic equations in cylindrical domains with applications. Math. Meth. Appl. Sci. 10, 51-66.

[25] Mielke, A. 1991 Hamiltonian and Lagrangian Flows on Center Manifolds. Berlin: Springer-Verlag.

[26] Moser, J. 1976 Periodic orbits near an equilibrium and a theorem by Alan Weinstein. Commun. Pure Appl. Math. 29, 727-747.

[27] Pego, R. L. \& Quintero, J. 2002 A host of traveling waves in a model of threedimensional water-wave dynamics. J. Nonlinear Sci. 12, 59-83.

[28] Plotnikov, P. I. 1980 Solvability of the problem of spatial gravitational waves on the surface of an ideal fluid. Sov. Phys. Dokl. 25, 170-171.

[29] ReEder, J. \& Shinbrot, M. 1981 Three dimensional nonlinear wave interaction in water of constant depth. Nonlinear Analysis TMA 5, 303-323.

[30] SCHMIDT, D. S. 1974 Periodic solutions near a resonant equilibrium of a Hamiltonian system. Celest. Mech. 9, 81-103.

[31] Stoker, J. J. 1957 Water Waves: The Mathematical Theory with Applications. New York: Interscience.

[32] Weinstein, A. 1973 Normal modes for nonlinear hamiltonian systems. Invent. Math. 20, 47-57.

[33] Wilton, J. R. 1915 On ripples. Phil. Mag. 29, 688-700. 